\title{
Dose-Response-Time Data Analysis: An Underexploited Trinity
}

\author{
Johan Gabrielsson, Robert Andersson, Mats Jirstrand, and Stephan Hjorth \\ Division of Pharmacology and Toxicology, Department of Biomedical Sciences and Veterinary Public Health, Swedish University of \\ Agricultural Sciences, Uppsala, Sweden (J.G.); Fraunhofer-Chalmers Centre, Gothenburg, Sweden (R.A., M.J.); Pharmacilitator AB, \\ Vallda, Sweden (S.H.); and Department of Molecular and Clinical Medicine, Institute of Medicine, Sahlgrenska Academy at Gothenburg \\ University, Gothenburg, Sweden (S.H.)
}

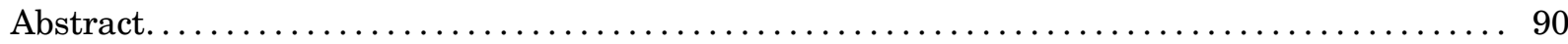

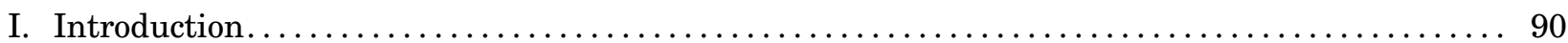

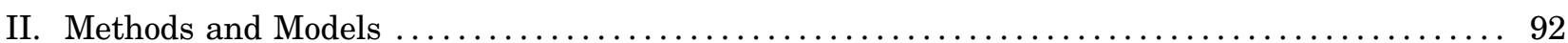

A. What Can Be Learned from Response-Time Data Patterns? . . . . . . . . . . . . . . . 92

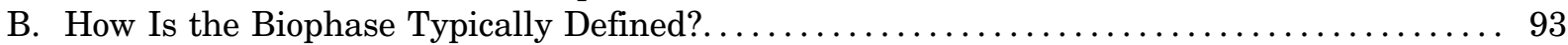

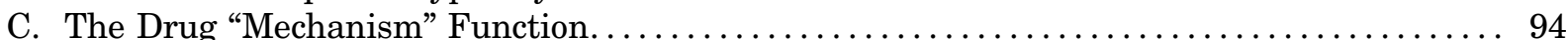

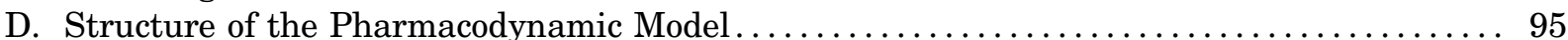

E. Response-Time Patterns Impact Model Structure............................... 95

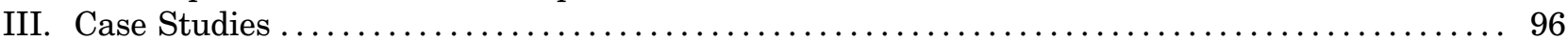

A. Case Study 1: Dose-Response-Time Analysis of Nociceptive Response . . . . . . . . . . . 96

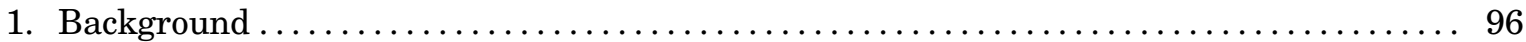

2. Models, Equations, and Exploratory Analysis ........................ 96

3. Results and Conclusions with Respect to Dose-Response-Time Analysis............ 99

4. Pharmacodynamic Interpretation and Comments ........................ 99

B. Case Study 2: Dose-Response-Time Analysis of Locomotor Stimulation. .............. 99

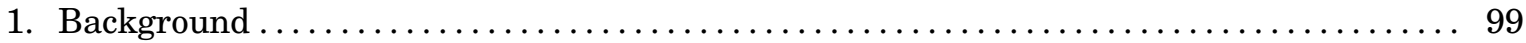

2. Models, Equations, and Exploratory Analysis ........................ 99

3. Results and Conclusions with Respect to Dose-Response-Time Analysis............ 101

4. Pharmacodynamic Interpretation and Comments ......................... 101

C. Case Study 3: Dose-Response-Time Analysis of Functional Adaptation ................. 102

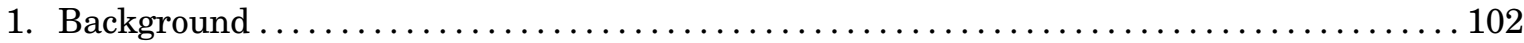

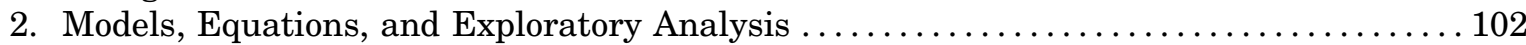

3. Results and Conclusions with Respect to Dose-Response-Time Analysis............ 103

4. Pharmacodynamic Interpretation and Comment ......................... 103

D. Case Study 4: Dose-Response-Time Analysis of Antipsychotic Effects (Brief Psychiatric

Rating Scale Scores) .............................................. 103

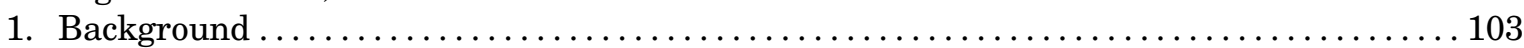

2. Models, Equations, and Exploratory Analysis ........................... 104

3. Results and Conclusions with Respect to Dose-Response-Time Analysis. . . . . . . . . . . 105

4. Pharmacodynamic Interpretation and Comments ........................... 106

E. Case Study 5: Dose-Response-Time Analysis of Bacterial Count . . . . . . . . . . . . . 106

1. Background ................................................. 106

2. Models, Equations, and Exploratory Analysis ......................... 106

3. Results and Conclusions with Respect to Dose-Response-Time Analysis............ 106

Address correspondence to: Johan Gabrielsson, Division of Pharmacology and Toxicology, Department of Biomedical Sciences and Veterinary Public Health, Swedish University of Agricultural Sciences, Box 7028, SE-750 07 Uppsala, Sweden. E-mail: johan. gabrielsson@slu.se

This work was funded in part through the Marie Curie Seventh Framework Programme People Initial Training Networks European Industrial Doctorate Project [Innovative Modeling for Pharmacological Advances through Collaborative Training Grant No. 316736] and by the Swedish Foundation for Strategic Research [(both to R.A. and M.J.)].

https://doi.org/10.1124/pr.118.015750. 
4. Pharmacodynamic Interpretation and Comments $\ldots \ldots \ldots \ldots \ldots \ldots \ldots \ldots \ldots \ldots \ldots \ldots$

F. Case Study 6: Dose-Response-Time Analysis of Cortisol-Adrenocorticotropic Hormone

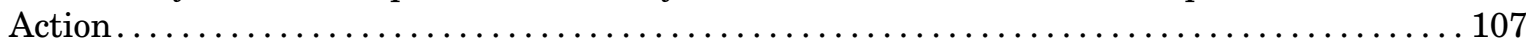

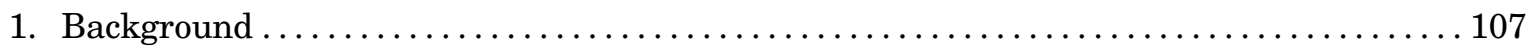

2. Models, Equations, and Exploratory Analysis ......................... 107

3. Results and Conclusions with Respect to Dose-Response-Time Analysis............. 107

4. Pharmacodynamic Interpretation and Comments ......................... 108

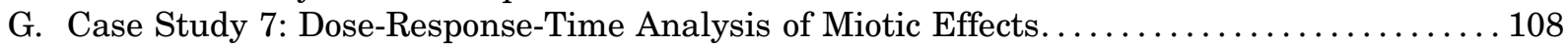

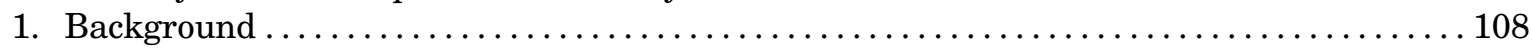

2. Models, Equations, and Exploratory Analysis .......................... 109

3. Results and Conclusions with Respect to Dose-Response-Time Analysis.............. 109

4. Pharmacodynamic Interpretation and Comments ........................ 109

H. Case Study 8: Meta-Analysis of Dose-Response-Time Data. ...................... 110

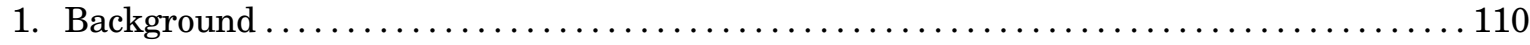

2. Results and Conclusions with Respect to Dose-Response-Time Analysis. . . . . . . . . 110

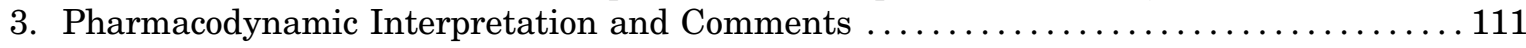

I. Overall Conclusions of the Case Studies................................. 111

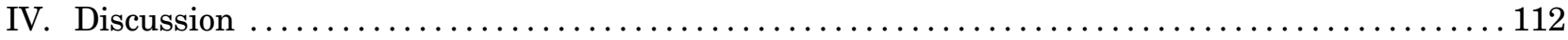

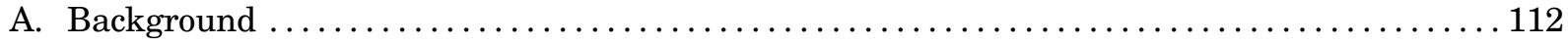

B. What Do Different Doses, Routes, and Rates of Input Add? ..................... 112

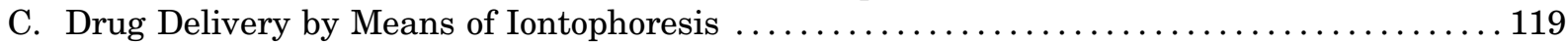

D. Permutations of Smolen's Model: The Kinetic-dynamic K-PD Rate of Change Model...... 120

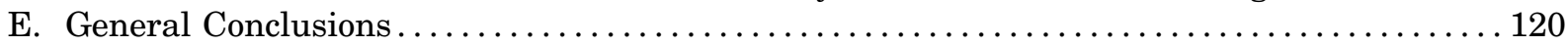

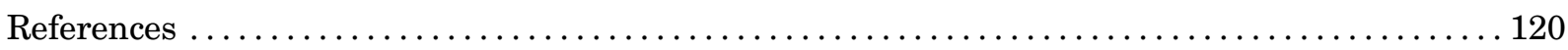

Abstract - The most common approach to in vivo pharmacokinetic and pharmacodynamic analyses involves sequential analysis of the plasma concentration- and response-time data, such that the plasma kinetic model provides an independent function, driving the dynamics. However, in situations when plasma sampling may jeopardize the effect measurements or is scarce, nonexistent, or unlinked to the effect (e.g., in intensive care units, pediatric or frail elderly populations, or drug discovery), focusing on the response-time course alone may be an adequate alternative for pharmacodynamic analyses. Response-time data inherently contain useful information about the turnover characteristics of response (target turnover rate, half-life of response), as well as the drug's biophase kinetics (biophase availability, absorption half-life, and disposition half-life) pharmacodynamic properties (potency, efficacy). The use of pharmacodynamic time-response data circumvents the need for a direct assay method for the drug and has the additional advantage of being applicable to cases of local drug administration close to its intended targets in the immediate vicinity of target, or when target precedes systemic plasma concentrations. This review exemplifies the potential of biophase functions in pharmacodynamic analyses in both preclinical and clinical studies, with the purpose of characterizing response data and optimizing subsequent study protocols. This article illustrates crucial determinants to the success of modeling dose-response-time (DRT) data, such as the dose selection, repeated dosing, and different input rates and routes. Finally, a literature search was also performed to gauge how frequently this technique has been applied in preclinical and clinical studies. This review highlights situations in which DRT should be carefully scrutinized and discusses future perspectives of the field.

\section{Introduction}

Pharmacological time-series data are typically rich in information. However, response-time series are often compared with plasma or blood exposure prior to any analysis of the connection between dose, response, and time data per se. Dose-response-time (DRT) data analysis is therefore an underexplored approach for quantification of the onset, intensity, and duration of a pharmacological response when information about the drug concentration is scarce or even totally lacking (Levy, 1971; Smolen, 1971a,b, 1976a,b, 1978; Smolen et al., 1972; Smolen and Weigand, 1973). Usually, plasma or tissue concentrations of drug are used as the "driver" of the pharmacological response. However, plasma concentrations may be of marginal value in situations with difficult or unethical sampling (e.g., pediatric or frail elderly populations), when due to local

ABBREVIATIONS: ACTH, adrenocorticotropic hormone; AUC, area under the curve; BPRS, Brief Psychiatric Rating Scale; CTC, circulating tumor cell; CV, coefficient of variation; DRT, dose-response-time; FFA, free fatty acid; $\mathrm{ID}_{50}, 50 \%$ of the maximum drug-induced inhibitory effect of the inhibitory drug mechanism function; K-PD, kinetic-dynamic model; NiAc, nicotinic acid; PTH, parathyroid hormone; $\mathrm{SD}_{50}$, biophase amount at $50 \%$ of maximum drug-induced effect. 
administration exposure at the pharmacological target precedes plasma concentrations (e.g., dermal or pulmonary applications, iontophoretic techniques), or in the case of extreme or unusual concentration-time profiles (e.g., the presence of active or interactive metabolites, mixture kinetics, or oligonucleotides). If the (pharmacological) biomarker response follows a time series, that time series normally contains useful information about target behavior [e.g., target turnover rates and halflives $\left(t_{1 / 2}\right)$ ] and drug properties [e.g., $\mathrm{ED}_{50}$ values, rate constants]. In some cases, the time course of drug molecules in the biophase (i.e., immediately adjacent to the target) may also reveal itself in the onset, intensity, and duration of response when the input rate to the biophase becomes the rate-limiting step. The biophase kinetics frequently differ from the plasma kinetics of a drug, particularly for peripherally placed targets and for slowly developing responses. Ideally, the in vivo drug response-time course is made up of three fundamental components: 1) the time course of drug molecules at the target (with plasma concentrations often used as a proxy and driver of the "drug mechanism" function), 2) the concentration-response relationship that contains potency and efficacy of the drug, and 3) the turnover of the target (or drug-target complex) as such. Thus, the underlying assumption in DRT analysis is that the time course of a drug at its target can be represented by a biophase function -in the absence of actual biophase concentrations of the drug. Is it then possible to deconvolute the biophase time course based on information about dose, rate, and route of administration coupled to the pharmacodynamic response-time courses? Figure 1 schematically shows the differences between the plasma concentrationtime (exposure)-driven pharmacological exploration and an analysis using biophase-driven response-time courses.

The exact driver of a pharmacological response will always be an approximation, independently of whether it is the concentration in plasma, in a tissue, or in a hypothetical biophase. However, it is important to remember that upon repeated dosing, the concentrations in plasma, tissue, and biophase are at equilibrium. This is not to say that the concentrations in the various matrices have to be equal, but their relative proportions are constant at equilibrium. To facilitate analysis of DRT data, pivotal parts of the pharmacological time course are summarized and compared with certain pharmacodynamic parameters in Table 1.

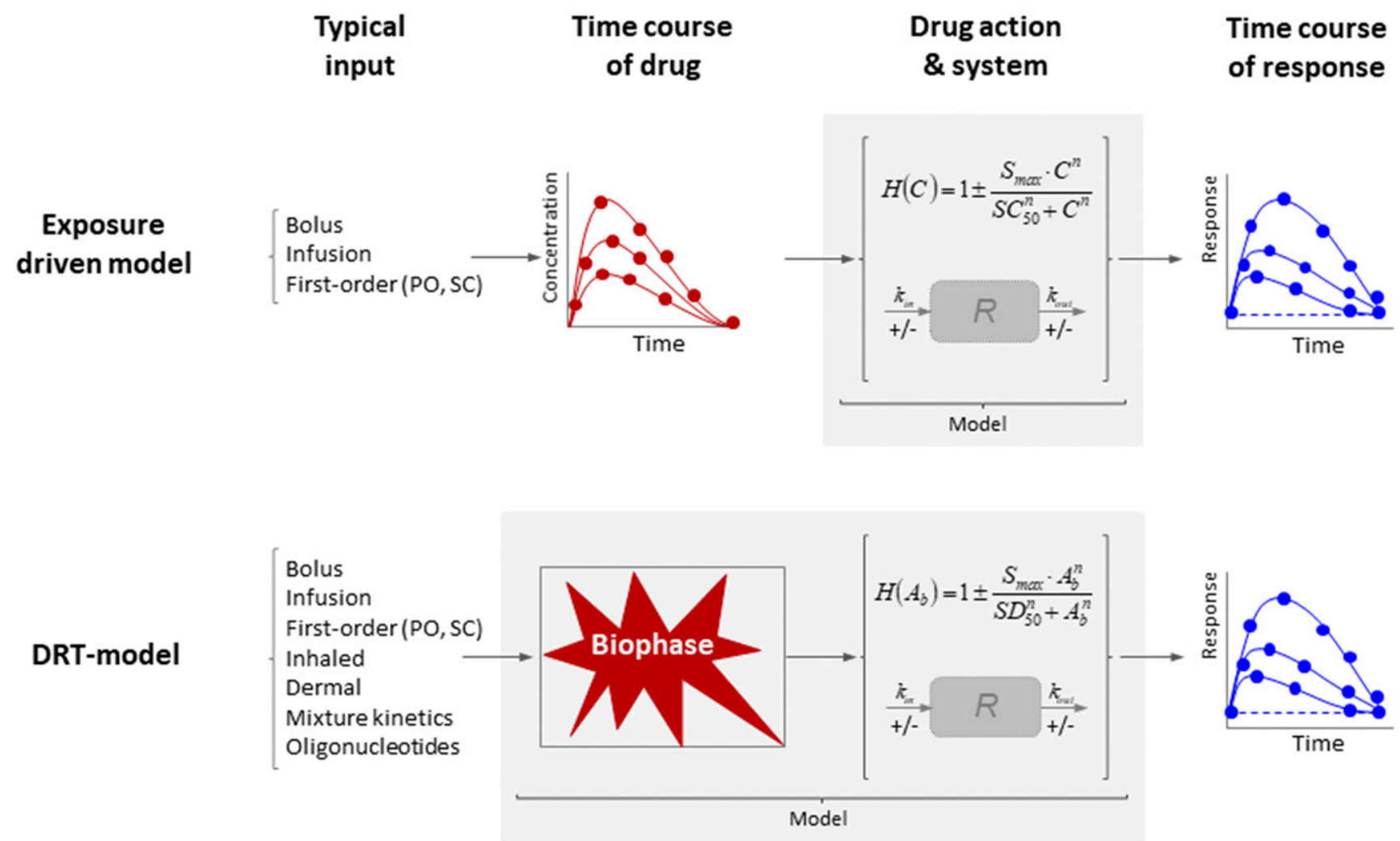

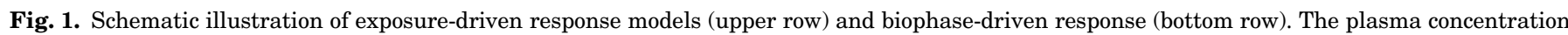

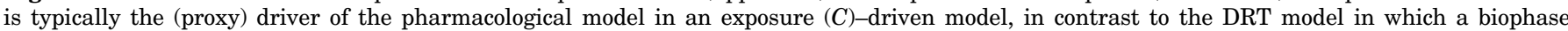

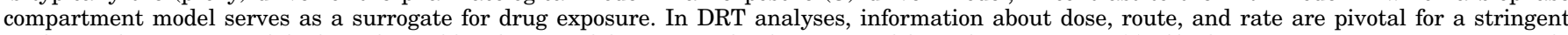

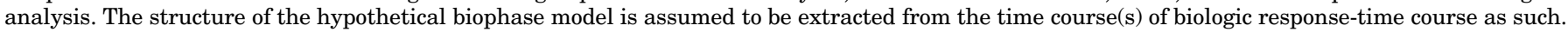

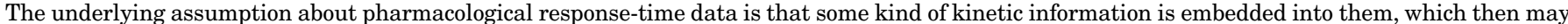

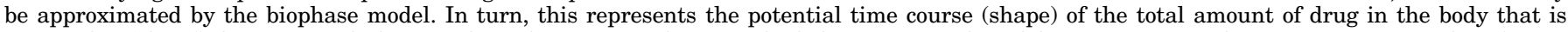

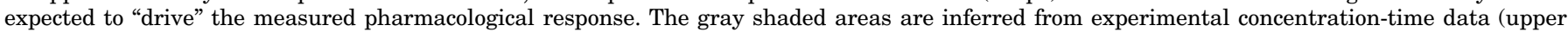

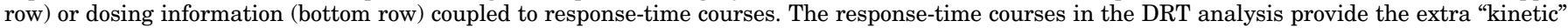
information to the model that concentration-driven pharmacodynamic models do not need. PO, per oral; SC, subcutaneous. 
TABLE 1

Overview of in vivo drug response phases, features of data, parameters, and case studies

\begin{tabular}{|c|c|c|c|}
\hline Phase & Features in Data & Parameter & Case Study \\
\hline A: baseline & $\begin{array}{l}\text { Stable, variable, oscillating, or drifting } \\
\text { (e.g., due to disease) }\end{array}$ & $R_{0}, k_{\text {in }}, k_{\text {out }}$ & $1,2-8$ \\
\hline B: time delay & $\begin{array}{l}\text { Time delay between expected plasma peak } \\
\text { concentration and peak of response }\end{array}$ & $k_{\text {out }}, K, K_{\mathrm{a}}$ & $1-6$ \\
\hline $\mathrm{C}$ : onset of action & $\begin{array}{l}\text { Concave or convex rise of response (onset of action); } \\
\text { overshoot during onset }\end{array}$ & $\begin{array}{l}k_{\text {out }}, K, K_{\mathrm{a}} \text {, number of transit } \\
\quad \text { compartments, } k_{\text {tol }}\end{array}$ & $1-7$ \\
\hline D: peak shift & $\begin{array}{l}\text { Peak shift or not; a shift suggests a nonlinear } \\
\text { stimulation/inhibition of action }\end{array}$ & $S_{\max } / I_{\max }, \mathrm{SD}_{50} / \mathrm{ID}_{50}, n$ & $1-6,8$ \\
\hline $\begin{array}{l}\text { E: response maximum } \\
\text { or minimum }\end{array}$ & $\begin{array}{l}\text { May indicate a nonlinear drug action or physiologic } \\
\text { limit; the same with an inhibitory drug action }\end{array}$ & $\begin{array}{l}S_{\text {max }} / I_{\text {max }}, \mathrm{SD}_{50} / \mathrm{ID}_{50}, n \\
\text { physiologic limit }\end{array}$ & $1-5,7,8$ \\
\hline $\mathrm{F}$ : return to baseline & $\begin{array}{l}\text { Different dose routes may reveal absorption } \\
\text { rate-limited elimination of drug from biophase; } \\
\text { rebound during washout }\end{array}$ & $k_{\text {out }}, K, K_{\mathrm{a}}, k_{\mathrm{tol}}$ & $1-3,5-8$ \\
\hline
\end{tabular}

This review aims to introduce the basic concepts of DRT analysis, anchor the concepts onto real-life case studies highlighting typical data patterns and designs of pharmacological studies in which DRT data analyses have proven to be useful, and finally, compile relevant literature about DRT data analysis.

The basic concepts of DRT analysis are introduced and illustrated by eight previously published case studies, each of which exemplifies different disease areas. The raw data for case studies 1-7 are available in a spreadsheet format (Gabrielsson and Weiner, 2010). The eighth case study is aimed at demonstrating how data from several studies in a drug discovery project can be merged and analyzed simultaneously (Andersson et al., 2017). This latter meta-analysis utilizes data from multiple dose provocations with nicotinic acid (NiAc; dose, route, rate, and mode), as well as two intertwined biomarkers (plasma fatty acids and insulin) in a large population of control and disease model rats. Finally, we searched the literature for studies in which DRT data analyses have been successfully performed. The result of this exploration is tabulated and collected in the list of references, which may then serve as a repository of relevant DRT literature.

\section{Methods and Models}

\section{A. What Can Be Learned from Response-Time Data Patterns?}

What may be extracted from time-series assessments of pharmacological responses? Figure 2 shows some typical features to look out for when analyzing DRT data after intravenous and subcutaneous dosing (Gabrielsson and Hjorth, 2016). These features include the following (to note, letters in parentheses refer to their

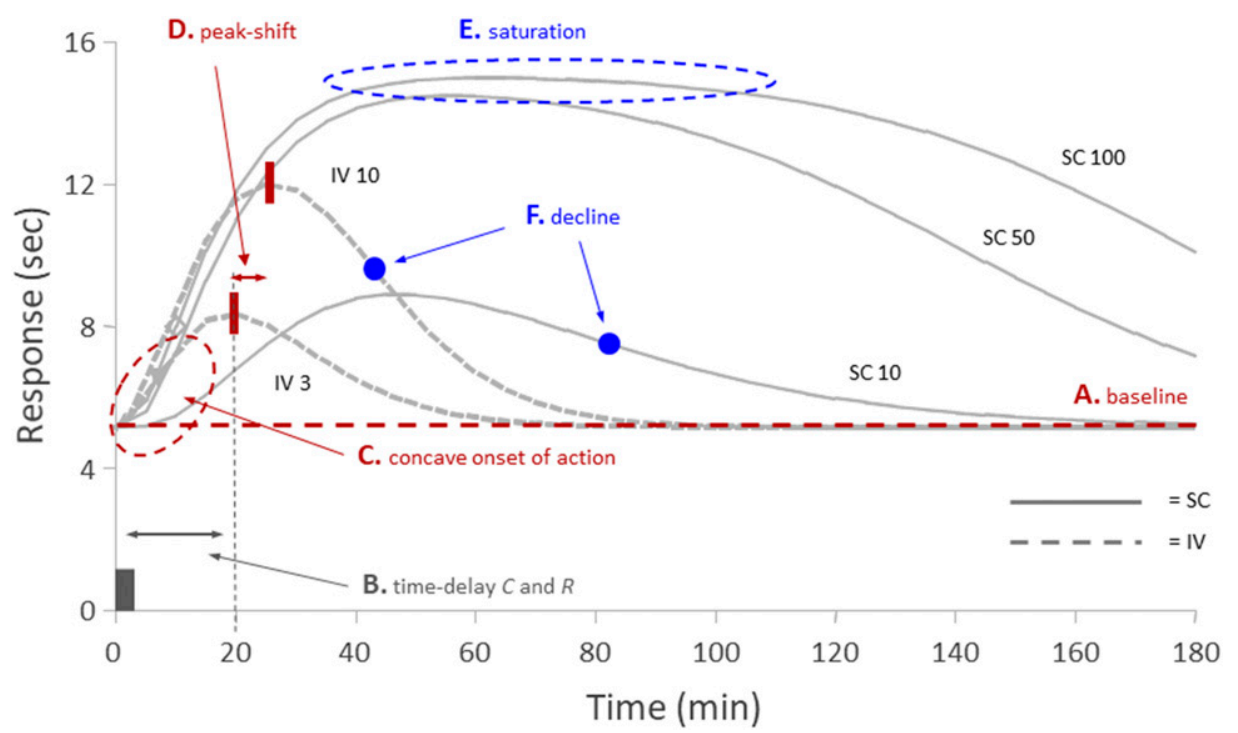

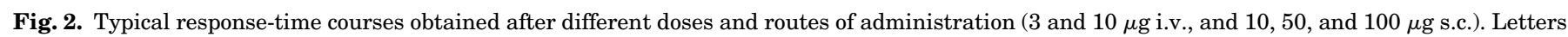

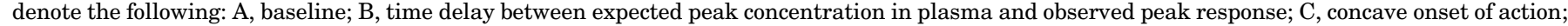

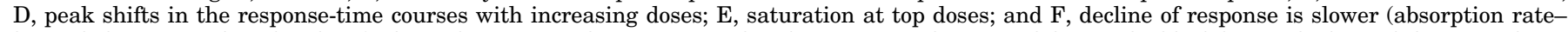

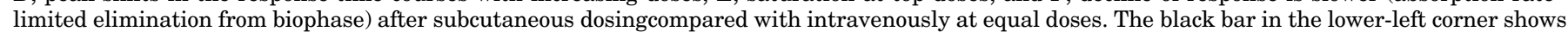

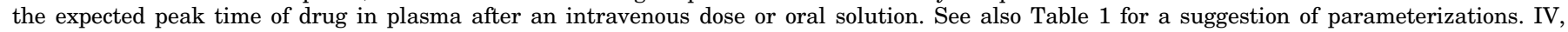
intraveonous; SC, subcutaneous. 
corresponding letters in Fig. 2): baseline level (A; made up from the ratio of turnover rate to fractional turnover rate of response; i.e., decay rate of response), time delay between expected plasma concentration peak and peak of response (B; may be due to indirect pharmacological action), onset of action ( $\mathrm{C}$; delayed onset may indicate a cascade of events upstream prior to biomarker compartment), peak shifts with increasing doses (D; indication of saturable stimulation), saturation of response (E; due to nonlinear drug mechanism or physiologic limit), and postpeak decline of response ( $\mathrm{F}$; the $10 \mu \mathrm{g}$ intravenous and subcutaneous time courses in Fig. 2 differ due to absorption rate-limited elimination after subcutaneous administration). Each portion and phase of the curves has its own story to tell with respect to drug properties, system properties, and biophase kinetics (Table 1).

\section{B. How Is the Biophase Typically Defined?}

A requirement for a robust description of the biophase model is that dose, rate, route, and mode of administration are known. The dose information combined with response-time courses bracket the biophase structure (Fig. 1, bottom row). If only response-time data are known, the individual contribution of input from drug administration and of biophase function cannot be separated. The biophase is a replacement of any possible time course of drug that "drives" the pharmacological effect and may be viewed as a first-generation effect-compartment approach. The biophase function represents the time course of drug in the body deconvoluted from response-time data necessary to drive the onset, intensity, and duration of the pharmacological response. The more remote and inaccessible the target (e.g., nuclear receptors) or the slower the turnover of response, often the slower the rate of its presentation and loss and the longer the action of drug at the target site. The transfer of the drug molecule to and from the target as well as the affinity for the target are elements (drug properties) separated from the turnover properties of the target as such and accompanying postreceptor events (biologic properties). It should be noted that drug and biologic properties jointly impact and make up the onset, intensity, and duration of a pharmacological response. Since the pharmacological response is a consequence of the two, it is reasonable to assume that the time course of response also contains some kind of kinetic information, which is governed by the biophase compartment. The biophase compartments in Fig. 3 are assumed to mimic that part of the pharmacological time course. The biophase kinetics will, to a varying extent, depend on the dose, the input rate (or rate of absorption of drug), and the route of administration, let alone the quality of response data.

The input/output of the biophase is assumed to make up all absorption and disposition processes that a drug may undergo until it hits the pharmacological target. Even though a drug was given as a bolus dose into plasma, the rise and fall of the pharmacological response may reveal a deviating pattern if the target is well separated from the plasma compartment and or the biophase exposure or response develops slowly. The biophase is meant to capture the rate-limiting step in the absorption and disposition of drug causing the necessary shape of exposure time at target.

The mathematical functions of the biophase amount $\left(A_{\mathrm{b}}\right)$ can be expressed as integrated solutions (eq. 1; top, bolus input; middle, first-order input/output; bottom, zero-order rate input):

$$
\left\{\begin{array}{c}
A_{\mathrm{b}}=D_{\mathrm{iv}} \cdot\left[e^{-K \cdot t}\right] \\
A_{\mathrm{b}}=\frac{K_{\mathrm{a}} \cdot F \cdot D_{\mathrm{ev}}}{\left(K_{\mathrm{a}}-K\right)}\left[e^{-K \cdot\left(t-t_{\mathrm{lag}}\right)}-e^{-K_{\mathrm{a}} \cdot\left(t-t_{\mathrm{lag}}\right)}\right] \\
A_{\mathrm{b}}=\frac{R_{\mathrm{in}}}{K}\left[1-e^{-K \cdot T_{\mathrm{inf}}}\right] \cdot e^{-K \cdot t^{\prime}}
\end{array}\right.
$$

$D_{\text {iv }}$ is the intravenous bolus dose, $K$ is the elimination rate constant, $K_{\mathrm{a}}$ is the absorption rate constant, $F^{*}$ is the biophase availability, $D_{\text {ev }}$ is the extravascular dose, $t_{\text {lag }}$ is the lag time, and $R_{\text {in }}$ is the rate of infusion. The biophase availability is the fraction of the dose that reaches the biophase relative to an intravenous dose, as approximated from pharmacological response data. This new parameter is different from the bioavailability, which is the fraction of the dose reaching the systemic circulation intact upon extravascular dosing. The biophase availability may be greater than unity (1) for a compound with pharmacologically active metabolites or saturable action.

The bolus, first-order input, and zero-order input can also be given as solutions of differential equations of a linear first-order system (eq. 2; second line from top, bolus input; second line from bottom, first-order input/output; and bottom, zero-order rate input):

$$
\left\{\begin{array}{l}
\frac{d A_{\mathrm{b}}}{d t}=\text { Input rate }- \text { output rate } \\
\frac{d A_{\mathrm{b}}}{d t}=-K \cdot A_{\mathrm{b}} \\
\frac{d A_{\mathrm{b}}}{d t}=k_{\mathrm{a}} \cdot F \cdot D_{\mathrm{ev}} \cdot e^{-K_{\mathrm{a}} \cdot t}-K \cdot A_{\mathrm{b}} \\
\frac{d A_{\mathrm{b}}}{d t}=\text { Input rate }-K \cdot A_{\mathrm{b}}
\end{array}\right.
$$

For nonlinear elimination, the three input modes (bolus, first-order input, and zero-order input) become the following (eq. 3):

$$
\left\{\begin{aligned}
\frac{d A_{\mathrm{b}}}{d t} & =-\frac{V_{\mathrm{max}} \cdot A_{\mathrm{b}}}{K_{\mathrm{m}}+A_{\mathrm{b}}} \\
\frac{d A_{\mathrm{b}}}{d t} & =K_{\mathrm{a}} \cdot F \cdot D_{\mathrm{ev}} \cdot e^{-K_{\mathrm{a}} \cdot t}-\frac{V_{\max } \cdot A_{\mathrm{b}}}{K_{\mathrm{m}}+A_{\mathrm{b}}} \\
\frac{d A_{\mathrm{b}}}{d t} & =\text { Input }-\frac{V_{\max } \cdot A_{\mathrm{b}}}{K_{\mathrm{m}}+A_{\mathrm{b}}}
\end{aligned}\right.
$$




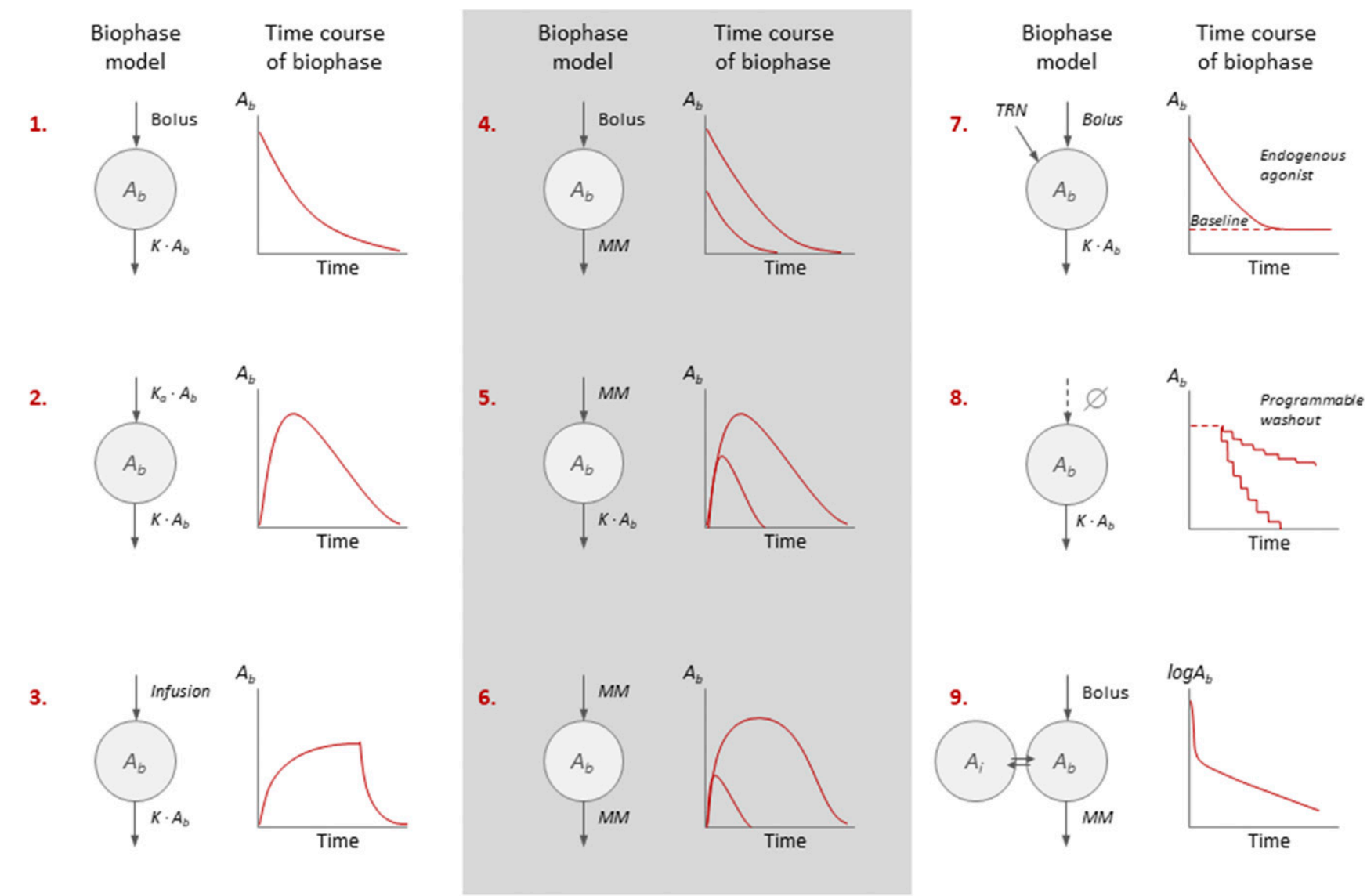

Fig. 3. Schematic illustration of different biophase models used for DRT analysis. Numbers indicate the following: (1) Biophase approximated by bolus administration and first-order elimination. (2) First-order input/output of the biophase. (3) Constant (zero-order) rate input followed by washout. (4) Bolus plus Michaelis-Menten (saturable) elimination. (5) Saturable absorption coupled to first-order elimination (note the peak shift). (6) Saturable absorption-elimination from biophase. (7) Bolus on top of endogenous ligand production. Baseline shows endogenous ligand concentration. (8) Stepdown input to biophase simulating longer half-lives for rapidly eliminated compounds (e.g., NiAc). (9) Biphasic decline in the biophase after a bolus dose. TRN, turnover.

For saturable input and output, the differential equations become the following (eq. 4):

$$
\left\{\begin{array}{c}
\frac{d D_{\mathrm{ev}}}{d t}=-\frac{K_{\mathrm{a}, \mathrm{max}} \cdot D_{\mathrm{ev}}}{K_{\mathrm{a}, \mathrm{m}}+D_{\mathrm{ev}}} \\
\frac{d A_{\mathrm{b}}}{d t}=F \cdot \frac{K_{\mathrm{a}, \max } \cdot D_{\mathrm{ev}}}{K_{\mathrm{a}, \mathrm{m}}+D_{\mathrm{ev}}}-\frac{V_{\mathrm{max}} \cdot A_{\mathrm{b}}}{K_{\mathrm{m}}+A_{\mathrm{b}}}
\end{array}\right.
$$

where $D_{\text {ev }}$ is the extravascular dose variable at the application site, $K_{\mathrm{a}, \max }$ is the maximum absorption rate, $K_{\mathrm{a}, \mathrm{m}}$ is the Michaelis-Menten constant (amount) at the halfmaximal absorption rate, and $F^{*}$ is the biophase availability. Again, the biophase availability $F^{*}$ is the fraction of the dose that reaches the biophase relative to intravenous dosing and is derived from pharmacological response-time data. Note that the biophase availability is not synonymous with bioavailability obtained from intravenous and oral dosing data. The biophase availability may be contaminated by active or interactive metabolites, antagonistic or synergistic action, feedback, and so forth and is a parameter derived from response-time data as such only.

Even though all of a bolus dose or a fraction (biophase availability) of an extravascular dose reaches the hypothetical biophase, only a small amount of drug is typically likely to reach its biologic target. The remainder will distribute nonspecifically to other body regions. The shape of the hypothetical biophase function is deconvoluted from the response-time data. As a result, it is not rational to assume that a hypothetical plasma time course will result from dividing the time course of the biophase by the volume of distribution. We therefore discourage any transformation of $A_{\mathrm{b}}$ to plasma concentration-time courses by means of the volume of distribution $\left(V_{\mathrm{d}}\right)$. However, converting the biophase amount at $50 \%$ of maximum drug induced effect $\left(\mathrm{SD}_{50}\right)$ or $50 \%$ of the maximum drug-induced inhibitory effect of the inhibitory drug mechanism function $\left(\mathrm{ID}_{50}\right)$ to $\mathrm{EC}_{50}$ or $\mathrm{IC}_{50}$ with volume is still feasible when operating under equilibrium conditions. The biophase still represents the body, but the shape of the function mimics the target site.

\section{The Drug "Mechanism" Function}

The interface between the biophase kinetics and the pharmacological model is the drug mechanism function. The biophase kinetics drives a linear (eq. 5) or nonlinear 
(eq. 6) drug mechanism function either as a stimulatory process [parameterized with the proportionality constant in the linear drug mechanism function $(a)$ or the efficacy parameter of the drug mechanism function $\left(S_{\max }\right)$, the $\mathrm{SD}_{50}$ value, and the Hill exponent $\left.\left(n_{\mathrm{H}}\right)\right]$ or as an inhibitory process [parameterized with the proportionality constant in the linear (inhibitory) drug mechanism function (b) or the $I_{\max }, \mathrm{ID}_{50}$, and $\left.n_{\mathrm{H}}\right]$.

$$
\left\{\begin{array}{l}
S\left(A_{\mathrm{b}}\right)=1+a \cdot A_{\mathrm{b}} \\
I\left(A_{\mathrm{b}}\right)=1-b \cdot A_{\mathrm{b}}
\end{array}\right.
$$

The nonlinear stimulatory and inhibitory drug mechanism functions are as follows:

$$
\left\{\begin{array}{l}
S\left(A_{\mathrm{b}}\right)=1+\frac{S_{\max } \cdot A_{\mathrm{b}}^{n_{\mathrm{H}}}}{\mathrm{SD}_{50}^{n_{\mathrm{H}}}+A_{\mathrm{b}}^{n_{\mathrm{H}}}} \\
I\left(A_{\mathrm{b}}\right)=1-\frac{I_{\max } \cdot A_{\mathrm{b}}^{n_{\mathrm{H}}}}{\mathrm{ID}_{50}^{n_{\mathrm{H}}}+A_{\mathrm{b}}^{n_{\mathrm{H}}}}
\end{array}\right.
$$

Logarithmic and exponential drug mechanism functions may also be applied, provided that data support such an approach. The pharmacological response is driven by the drug mechanism function, which converts drug input (typically concentration or amount) to a pharmacological action.

\section{Structure of the Pharmacodynamic Model}

When the equilibrium between the biophase and the observed pharmacological response is rapid, the biophase function can be used to directly drive an analytical function of the response $E$. Equation 7 shows a stimulatory (upper row) and an inhibitory (bottom row) efficacy parameter of the Hill equation $\left(E_{\max }\right)$ model driven by the biophase amount $A_{\mathrm{b}}$.

$$
\left\{\begin{array}{c}
E=E_{0}+\frac{E_{\max } \cdot A_{\mathrm{b}}^{n_{\mathrm{H}}}}{\mathrm{ED}_{50}^{n_{\mathrm{H}}}+A_{\mathrm{b}}^{n_{\mathrm{H}}}} \\
E=E_{0}-\frac{I_{\max } \cdot A_{\mathrm{b}}^{n_{\mathrm{H}}}}{\mathrm{ID}_{50}^{n_{\mathrm{H}}}+A_{\mathrm{b}}^{n_{\mathrm{H}}}}
\end{array}\right.
$$

The model structure includes a baseline value $E_{0}$, the maximum effect $E_{\max }$ or $I_{\max }$, the dose at half-maximal effect $\mathrm{ED}_{50}$ or $\mathrm{ID}_{50}$, and the sigmoidicity factor $n_{\mathrm{H}}$. In this case, the pharmacological response model is somewhat similar to the previously presented drug mechanism function mentioned above.

When a drug acts on factors responsible for the buildup or loss of a pharmacological response, the kind of turnover model shown in Fig. 4 is typically applied. The drug either stimulates or inhibits the production of response [zero-order turnover rate $\left(k_{\text {in }}\right)$ ] or the loss term of response [first-order fractional turnover rate $\left.\left(k_{\text {out }}\right)\right]$. The drug directly impacts factors controlling the response $R$ such as $k_{\text {in }}$ and $k_{\text {out }}$. The drug

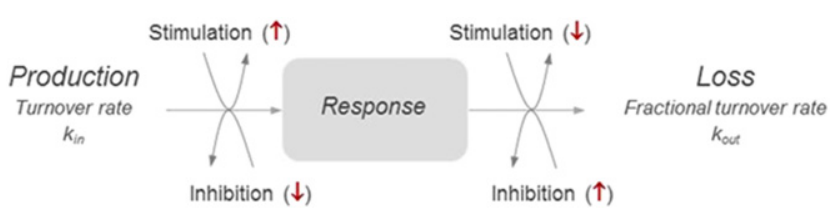

Fig. 4. Conceptual diagram of the turnover model (indirect response model) where drug either impacts the production of response $k_{\text {in }}$ or the loss of response $k_{\text {out }}$. The drug acts on factors controlling the level of response $R$. The red arrows show the rise or decrease in response as a consequence of drug action.

indirectly, via $k_{\text {in }}$ and $k_{\text {out }}$, affects the pharmacological response $R$ as such.

The drug mechanism function $\left[S\left(A_{\mathrm{b}}\right)\right.$ or $\left.I\left(A_{\mathrm{b}}\right)\right]$ is then incorporated into one of the response production or loss systems (see, Fig. 4) as follows:

$$
\left\{\begin{array}{l}
\frac{d R}{d t}=k_{\text {in }} \cdot S\left(A_{\mathrm{b}}\right)-k_{\text {out }} \cdot R \\
\frac{d R}{d t}=k_{\text {in }}-k_{\text {out }} \cdot S\left(A_{\mathrm{b}}\right) \cdot R \\
\frac{d R}{d t}=k_{\text {in }} \cdot I\left(A_{\mathrm{b}}\right)-k_{\text {out }} \cdot R \\
\frac{d R}{d t}=k_{\text {in }}-k_{\text {out }} \cdot I\left(A_{\mathrm{b}}\right) \cdot R
\end{array}\right.
$$

Equation 8 shows the four basic turnover systems with stimulation or inhibition of either turnover rate $k_{\text {in }}$, or on the fractional turnover rate $k_{\text {out }}$. These models may be adjusted depending on the particular pharmacological target and system studied. The onset of response may reflect a series of transduction compartments to capture a concave buildup of response. Other systems display oscillating time courses and may also require diurnal variations in turnover rate. A third may involve one or more amplification steps (Gabrielsson et al., 2000; Gabrielsson and Weiner, 2010). A more detailed account of biophase structure, drug mechanism, and turnover systems will be given for each case study.

\section{E. Response-Time Patterns Impact Model Structure}

We previously described "pattern recognition" as a graphical tool for delineating the structure of a pharmacological model (Gabrielsson and Hjorth, 2016). That article also discussed practical experimental design to highlight some pivotal guidelines. In brief, information on the baseline is central to model building. Significant information is also contained in the overall shape of the curve: potential time delays between plasma exposure and a pharmacological response, peak shifts in the response-time course with increasing doses (indicative of whether there is a linear or nonlinear drug mechanism function at play), and saturation at the maximum or minimum of response, which in combination with peak shifts is a strong indicator of the nonlinear nature of the drug mechanism function. This generic approach is likewise applicable 
to the DRT analysis we describe here. Figure 5 shows four common patterns in response-time curves and their tentative models (and case studies).

\section{Case Studies}

Eight case studies of previously published data are compiled and presented here to demonstrate the pros and cons of DRT analysis. This analysis contains eight case study datasets that were in part originally published in Pharmacokinetic and Pharmacodynamic Data Analysis: Concepts and Applications (first to fifth editions) by Gabrielsson and Weiner (2010, 2016) in 1994-2016 or in Gabrielsson et al. (2000) but have not been easily accessible to a larger audience; they are therefore included due to their availability and functionality. The experimental data of case studies 1-7 are available electronically as models, command files, and project files via https://dipublish.com/pkpd/. Data on cortisol-adrenocorticotropic hormone (ACTH) dynamics (Urquhart and $\mathrm{Li}, 1968$ ), locomotor activity (van Rossum and van Koppen, 1968), and Brief Psychiatric Rating Scale (BPRS) scores (Lewander et al., 1990) were scanned from the literature. Case study 8 is a summary of Kroon et al. (2017). Some of the figures and datasets have been used for teaching and at various conferences. The datasets were selected to illustrate response-time pattern and design feature points of view, rather than to compare models and drugs within specific therapeutic classes. Table 2 gives an overview of the background on each case study.

\section{A. Case Study 1: Dose-Response-Time Analysis of Nociceptive Response}

1. Background. This case study comprises pharmacological data obtained using two different routes of administration (intravenous and subcutaneous) to model and estimate pertinent screening parameters such as $\mathrm{SD}_{50}$, biophase availability, and turnover characteristics of the (analgetic) response, such as half-life. The underlying assumption is that the pharmacodynamic data convey information about the kinetics of the drug in the biophase (rate constants, biophase availability), which can be obtained simultaneously from DRT data. Since both intravenous and subcutaneous administration modes were applied, a new parameter (biophase availability) could be estimated.

A potential analgesic test compound was given at two dose levels ( 3 and $10 \mu \mathrm{g}$ ) via the intravenous route and at three dose levels $(10,50$, and $100 \mu \mathrm{g})$ subcutaneously. The time (in seconds) to respond to a noxious stimulus (laser beam challenge in the tail-flick test) was determined at different times after dosing. Response-time data were obtained from each dose level after an acute dose (Fig. 6).

2. Models, Equations, and Exploratory Analysis. The underlying biophase-coupled turnover model is shown in Fig. 6 (right). The biophase behaves as a
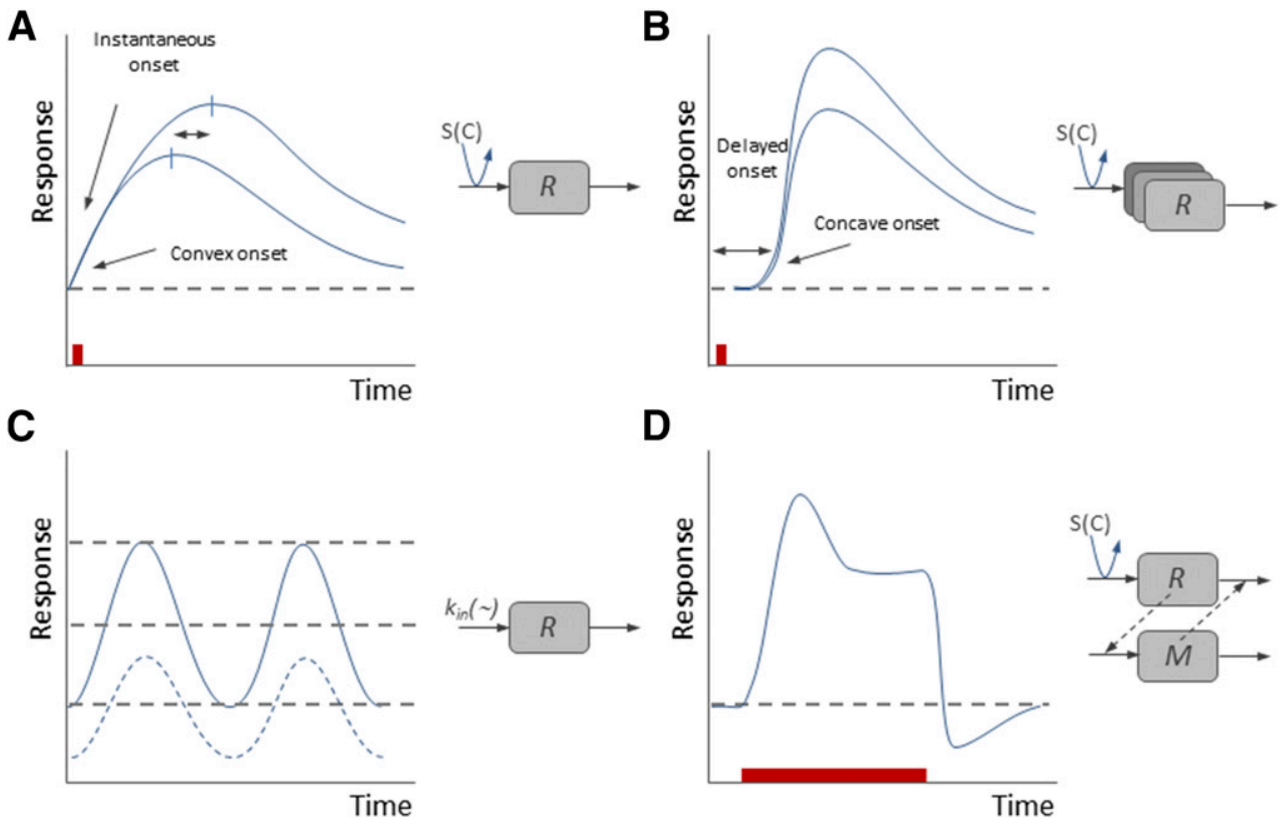

Fig. 5. Commonly encountered response-time courses and their corresponding potential turnover models. (A) Standard convex onset of action after stimulation of turnover rate $k_{\text {in }}$ after two doses (see also case study 1). (B) Concave onset of effect as a consequence of an early upstream stimulation of turnover rate at two doses followed by one or more transduction processes prior to the final response-generating compartment (see case study 2 on locomotor activity). (C) Diurnal variation in the turnover rate, resulting in an oscillating (baseline and) response-time course. The dashed oscillatory curve shows response after inhibitory drug intervention. (D) Feedback-regulated response. A period of drug-induced stimulation of response is followed by rebound (response-time course below the predose baseline level). The red horizontal bar in plot (D) shows the drug exposure period. The small red bars in the upper row plots show the expected peak time of drug in plasma (see also case studies 1-3 and 6). 


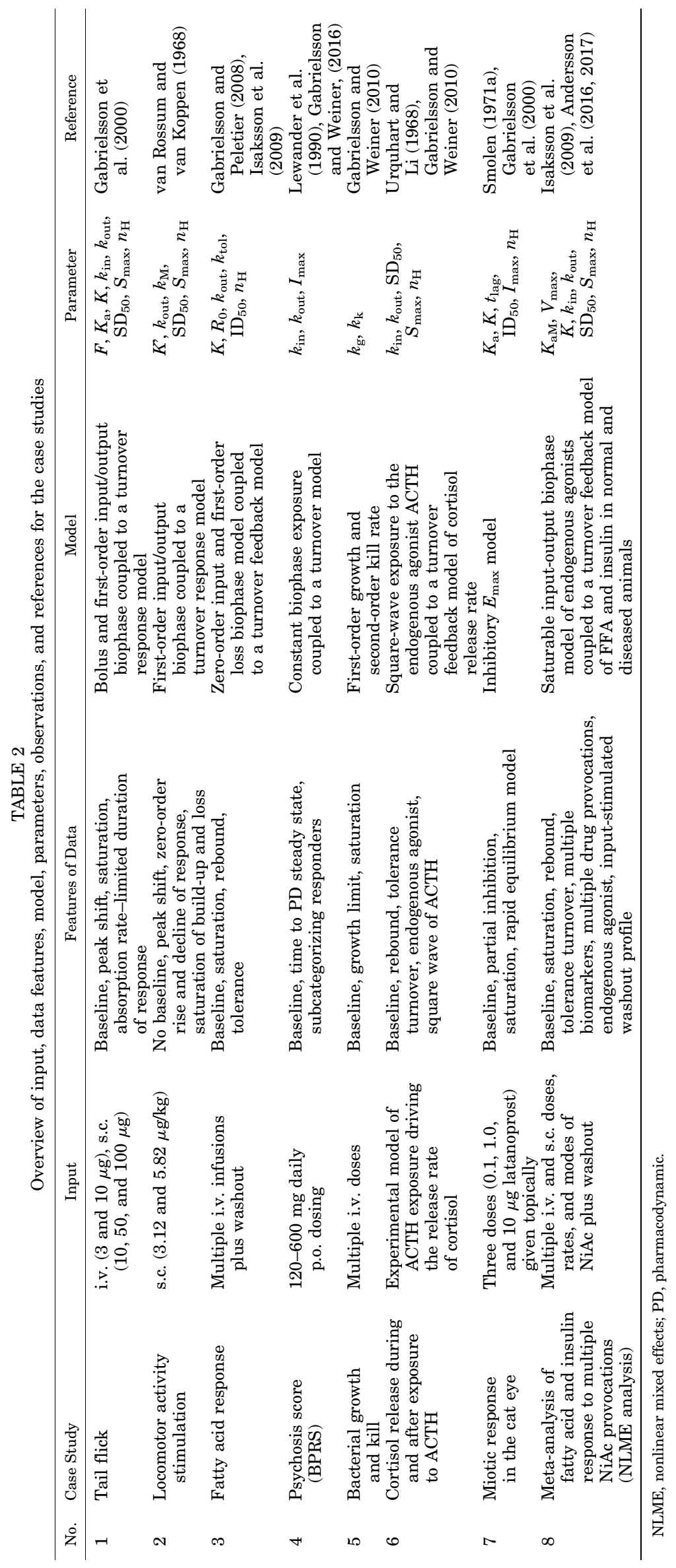




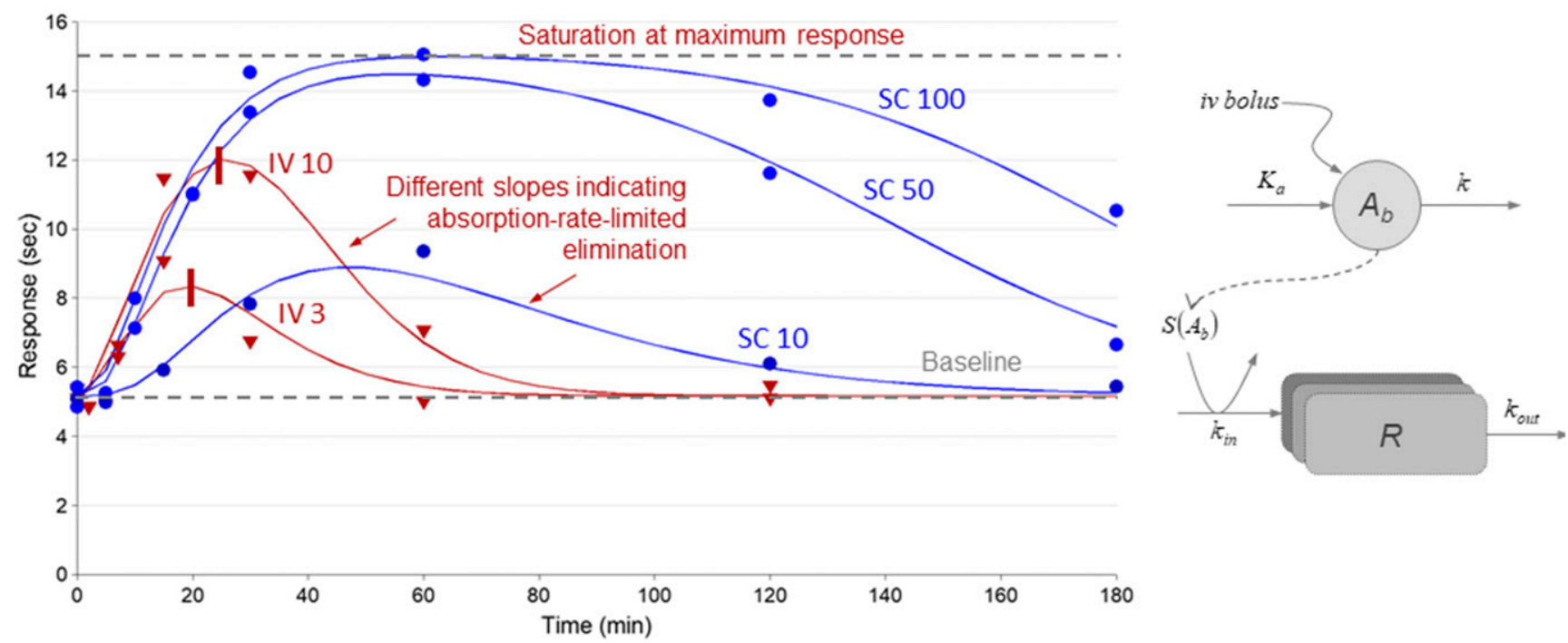

Fig. 6. (Left) Observed (symbols) and model-predicted (lines) response (tail flick) vs. time of drug X after different doses given by the intravenous (red) and subcutaneous (blue) routes. The subcutaneous doses were 10,50, and $100 \mu \mathrm{g}$, and the intravenous doses were 3 and $10 \mu \mathrm{g}$. Note the slow terminal decline of the $10 \mu \mathrm{g}$ subcutaneous dose compared with the $10 \mu \mathrm{g}$ intravenous dose, suggesting absorption rate-limited elimination. (Right) Schematic illustration of the biophase (top) and the turnover response (bottom) models. The drug in the biophase stimulates the production of the antinociceptive response. Also note that we have added a transit compartment to mimic the concave buildup of response. $K_{\mathrm{a}}$ denotes the first-order input rate constant, $A_{\mathrm{b}}$ is the biophase amount, $k$ is the first-order elimination rate constant, $S\left(A_{\mathrm{b}}\right)$ is the stimulatory "drug mechanism" function, $k_{\text {in }}$ is the turnover rate, $R$ is the measured (biomarker) response, and $k_{\text {out }}$ is the fractional turnover rate. IV, intraveonous; SC, subcutaneous.

one-compartment bolus model for intravenous data (eq. 9, top row) and as a first-order input-output model for subcutaneous dosing (eq. 9, bottom row). The biophase model then drives the stimulatory drug mechanism function (eq. 10), acting on a series of transit compartments that capture the slight concave onset of response in both intravenous and subcuteanous data (eq. 11). Drug action occurs via stimulation of the production of response (i.e., analgesia, resulting in a delayed reaction time to noxious stimuli). A zeroorder input and first-order input/output model governs the turnover of the response.

The amount of drug in the biophase compartment after intravenous and subcutaneous dosing is modeled with first-order kinetics assuming the volume is unity (eq. 9):

$$
\left\{\begin{array}{c}
A_{\mathrm{b}}=D_{\mathrm{iv}} \cdot\left[e^{-K \cdot t}\right] \\
A_{\mathrm{b}}=\frac{K_{\mathrm{a}} \cdot F \cdot D_{\mathrm{sc}}}{\left(K_{\mathrm{a}}-K\right)}\left[e^{-K \cdot\left(t-t_{\mathrm{lag}}\right)}-e^{-K_{\mathrm{a}} \cdot\left(t-t_{\mathrm{lag}}\right)}\right]
\end{array}\right.
$$

$D_{\text {iv }}$ and $D_{\text {sc }}$ denote the intravenous and subcutaneous doses, $K$ is the elimination rate constant, $K_{\mathrm{a}}$ is the absorption rate constant, and $F^{*}$ and $t_{\text {lag }}$ are the biophase availability and lag time, respectively. Note that there are no volume terms in eq. 10 for the biophase amount expressions. The stimulatory drug mechanism function driver is as follows:

$$
S\left(A_{\mathrm{b}}\right)=1+\frac{S_{\max } \cdot A_{\mathrm{b}}^{n_{\mathrm{H}}}}{\mathrm{SD}_{50}^{n_{\mathrm{H}}}+A_{\mathrm{b}}^{n_{\mathrm{H}}}}
$$

$S_{\max }, \mathrm{SD}_{50}$, and $n_{\mathrm{H}}$ denote the maximum drug-induced effect of the drug mechanism function, the dose generating $50 \%$ of maximum drug-induced response, and the sigmoidicity parameter, respectively. The turnover function of the pharmacological response with stimulation of turnover rate $k_{\text {in }}$ of $R_{1}$ is written as a series of transit compartments, which capture the concave onset of response.

$$
\left\{\begin{array}{c}
\frac{d R_{1}}{d t}=k_{\text {in }} \cdot S\left(A_{\mathrm{b}}\right)-K_{\text {out }} \cdot R_{1} \\
\frac{d R_{2}}{d t}=k_{\text {out }} \cdot R_{1}-K_{\text {out }} \cdot R_{2} \\
\frac{d R_{\text {obs }}}{d t}=k_{\text {out }} \cdot R_{2}-K_{\text {out }} \cdot R_{\text {obs }}
\end{array}\right.
$$

$k_{\text {in }}$ and $k_{\text {out }}$ are the turnover rate and the fractional turnover rate constants, respectively. The maximum drug-induced change $\Delta$ is obtained from eq. 12:

$\left\{\begin{array}{l}\Delta=R_{\mathrm{ss}}-R_{0}=R_{0} \cdot\left(1+E_{\max }\right)-R_{0}=R_{0} \cdot E_{\max } \\ 10=R_{0} \cdot E_{\max }=5 \cdot 2\end{array}\right.$

where $\Delta$ is derived from the absolute difference between baseline $R_{0}$ and $R_{\max }$. An approximate estimate of biophase availability $F^{*}$ can be obtained by area under the curve $\mathrm{AUC}_{E \mathrm{sc}} / \mathrm{AUC}_{E \text { iv }}$ for the $10 \mu \mathrm{g}$ intravenous and subcutaneous doses, which gives approximately $100 \%$. To note, when noncompartmental assessment of the biophase availability is used, the areas under the baseline have to be subtracted from each total $\mathrm{AUC}_{E}$. 
TABLE 3

Initial and final parameter estimates and their corresponding precision $(\mathrm{CV} \%)$

\begin{tabular}{lccr}
\hline \multicolumn{1}{c}{ Parameter } & Initial Estimate & Final Estimate & CV\% \\
\hline$K_{\mathrm{a}}\left(\min ^{-1}\right)$ & 0.01 & 0.018 & 8 \\
$K\left(\min ^{-1}\right)$ & 0.05 & 0.063 & 19 \\
$F^{*}$ & 1 & 0.86 & 11 \\
$k_{\text {out }}\left(\min ^{-1}\right)$ & 0.3 & 0.13 & 8 \\
$n_{\mathrm{H}}$ & 2 & 2.0 & 14 \\
$\mathrm{SD}_{50}(\mu \mathrm{g})$ & $1-10$ & 1.8 & 22 \\
$S_{\max }$ & 2 & 2.0 & 5 \\
\hline
\end{tabular}

Initial estimates of the $k_{\text {out }}$ parameter $\left(0.3 \mathrm{~h}^{-1}\right)$ were derived from the initial slope of the upswing of the response-time curve after the largest dose. The initial estimate of the $k_{\text {in }}$ parameter was derived from $k_{\text {out }}$ and the corresponding ratio of $k_{\text {in }} / k_{\text {out }}$. ID 50 was obtained by simulating the model with a number of realistic values of $\mathrm{SD}_{50}$ within the $0-10 \mu \mathrm{g}$ range.

3. Results and Conclusions with Respect to DoseResponse-Time Analysis. A high correlation between observed and estimated response-time data (Fig. 6), as well as well defined parameters with good precision (Table 3), is obtained by simultaneously fitting eqs. 911 to all five response-time courses.

Comparison of the intravenous and subcutaneous $10 \mu \mathrm{g}$ DRT data suggested absorption rate-limited elimination via the subcutaneous route. Thus, in spite of a higher initial response of the $10 \mu \mathrm{g}$ intravenous dose, the time course declined faster than the corresponding response-time course of a $10 \mu \mathrm{g}$ subcutaneous dose. The biophase availability was $86 \%$. The simulated biophase (dose) response relationship is shown in Fig. 7.

4. Pharmacodynamic Interpretation and Comments. Depending on the drug target involved, analgetic drug actions may involve central (spinal, supraspinal) as well as peripheral components (e.g., Sawynok and Liu, 2014). For the particular case described above, it is assumed that one of these compartments is the dominant antinociceptive biophase for compound X. The tail-flick response represents a classic model of acute thermal pain sensitivity considered to involve central (spinal/supraspinal) pathways. It might be suggested that in this case (regardless of whether the biophase target sites are fully occupied, and in the absence of further information on test compound properties), maximal analgesia via this particular target by compound $\mathrm{X}$ is attained. Moreover, the observed time course may represent a buildup of biophase exposure to reach this level and/or contributions from the actual biologic mechanism underlying the analgetic response monitored. In this regard, the protracted response curve seen with subcutaneous versus intravenous administration may suggest that compound $\mathrm{X}$ has properties that may limit absorption and potentially also penetration across blood-brain/spinal fluid barriers, thus influencing the biophase levels. This case thus illustrates the value of the integrated use of dose, response, and time

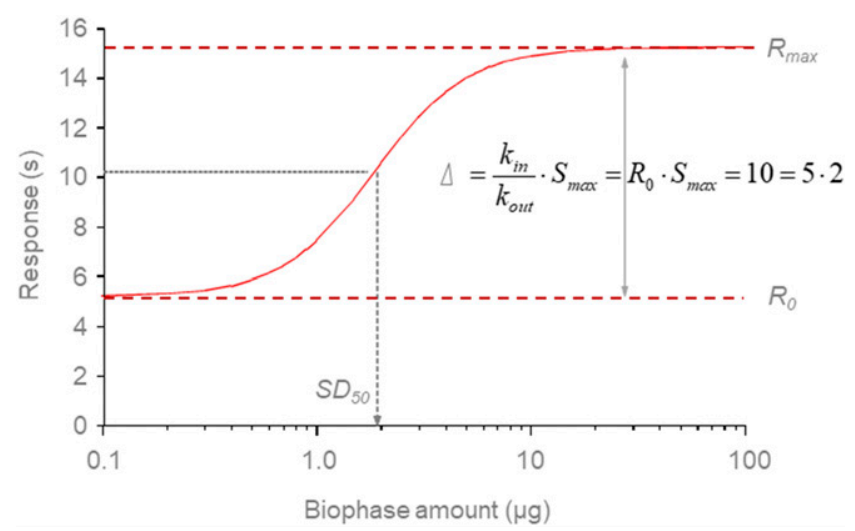

Fig. 7. Simulated response (tail flick) vs. biophase amount (dose) of compound $\mathrm{X}$. The baseline (vehicle treatment) response is about 5 seconds and the maximum drug-induced response is 10 seconds, resulting in a 15 -second response maximum in these settings.

data to arrive at biophase estimates with plausible precision.

\section{B. Case Study 2: Dose-Response-Time Analysis of Locomotor Stimulation}

1. Background. Response-time data on rat locomotor activity after intraperitoneal administration of dexamphetamine at two dose levels were obtained and digitized from van Rossum and van Koppen (1968). These data suit the purpose of DRT modeling, since the resolution is high, with adequate granularity in both the rise and decline in response, a clear peak shift with dose, and more than one dose level (assuming first-order input) used. A slight concavity was observed in the onset of response, followed by an apparently linear rise during the first 30 minutes after dosing. The initial rise in locomotor response superimposed for both doses and was followed by an abrupt change from rise to decline. The response to the higher dose peaked later than the response to the lower dose. The slope of the postpeak linear decline of response was independent of dose (3.12 and 5.62 $\mu \mathrm{g} / \mathrm{kg}$ ) or route of administration (intraperitoneal or intramuscular application; see van Rossum and van Koppen, 1968) (Fig. 8).

2. Models, Equations, and Exploratory Analysis. Figure 8 (right) shows the suggested biophase compartment models of this analysis. They also demonstrate the "duality of models" when two competing models are equally good representatives of the pharmacology. Note that the drug stimulatory action is assumed to act on the production of response.

Two biophase models were fitted: one approximating the input to the biophase as a bolus, and the other applying a first-order input (eq. 13), resulting in the functions where $A_{\mathrm{b}}$ denotes the biophase amount after subcutaneous dosing. The biophase availability $F^{*}$ was set equal to unity since no comparative data from intravenous dosing were available. 

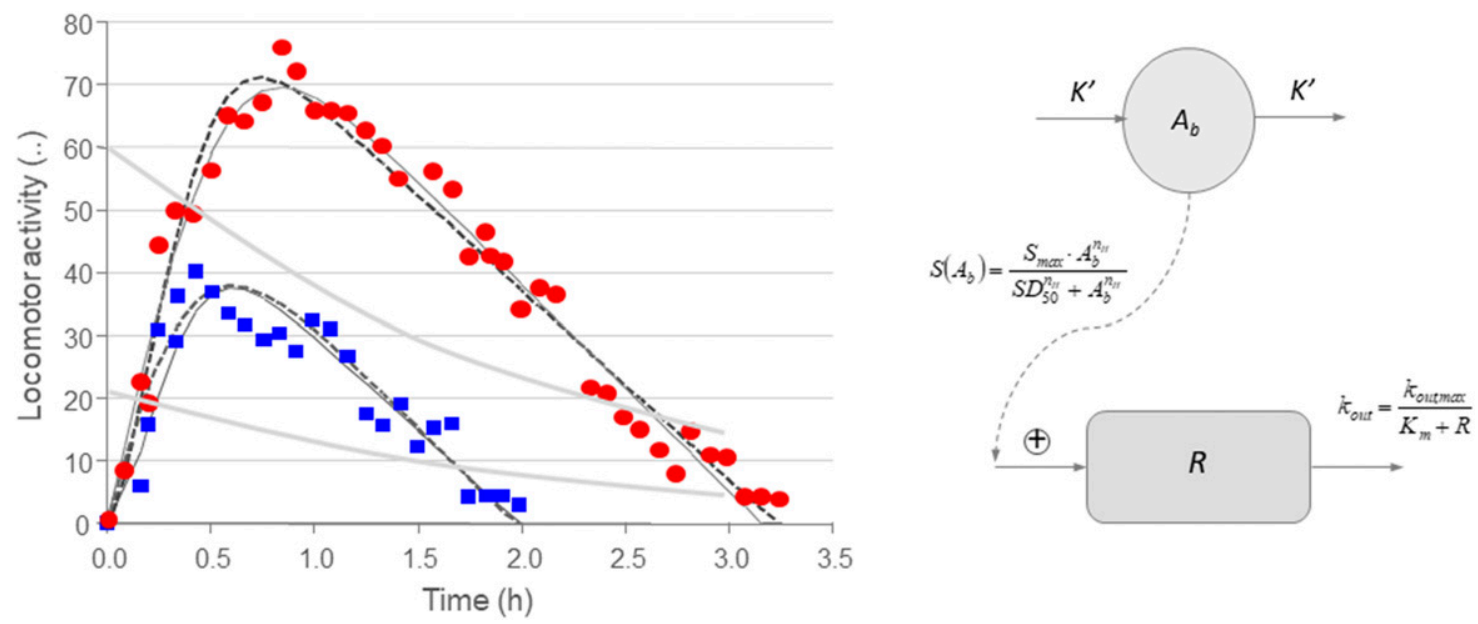

Fig. 8. (Left) Rat locomotor activity score (counts per minute) time data after dexamphetamine is given intravenously at 3.12 (blue symbols) or 5.62 $\mu \mathrm{g} / \mathrm{kg}$ doses (red symbols) (van Rossum and van Koppen, 1968). Note the apparently linear and parallel decline in response over time independently of dose and the peak shift to the right with increasing dose. A slight concave onset of action may also be discernible, particularly with the lower of the two doses. No saturation manifested as a plateau (flat horizontal) response-time region can be seen with the highest dose. Dashed lines represent the bolus input biophase model, and the solid lines represent the first-order input/output model. (Right) Conceptual schematic model of the DRT data. $K^{\prime}$ denotes the first-order input/output biophase rate constant and $S_{\max }, \mathrm{SD}_{50}$, and $n$ are the pharmacodynamic parameters of maximum efficacy, biophase amount at $50 \%$ of maximum efficacy, and the Hill-parameter, respectively. The constant term (1) in the definition of $S\left(A_{\mathrm{b}}\right)$ in eq. 14 could be eliminated because no baseline activity was observed in the data. Note the saturable loss of response given by the fractional turnover rate, $k_{\text {out }}(R)=k_{\text {out,max }} /\left(k_{\mathrm{M}}+R\right)$. Gray solid lines represent simulated plasma time courses of dexamphetamine at the two doses given.

$$
\left\{\begin{array}{c}
A_{\mathrm{b}}(t)=D \cdot e^{-K^{\prime} \cdot t} \\
A_{\mathrm{b}}(t)=F^{*} \cdot D \cdot K^{\prime} \cdot t \cdot e^{-K{ }^{\prime} t}
\end{array}\right.
$$

The stimulatory drug mechanism function is shown in eq. 14:

$$
\left\{\begin{array}{c}
S\left(A_{\mathrm{b}}\right)=\frac{S_{\mathrm{max}} \cdot A_{\mathrm{b}}^{n_{\mathrm{H}}}}{\mathrm{SD}_{50}^{n_{\mathrm{H}}}+A_{\mathrm{b}}^{n_{\mathrm{H}}}} \\
S\left(A_{\mathrm{b}}(0)\right)=0 \\
S\left(A_{\mathrm{b}}\right)=1+\frac{S_{\max } \cdot A_{\mathrm{b}}^{n_{\mathrm{H}}}}{\mathrm{SD}_{50}^{n_{\mathrm{H}}}+A_{\mathrm{b}}^{n_{\mathrm{H}}}} \\
S\left(A_{\mathrm{b}}(0)\right)=1
\end{array}\right.
$$

where $S_{\text {max }}, \mathrm{SD}_{50}$, and $n_{\mathrm{H}}$ correspond to the maximum drug-induced efficacy, potency, and Hill exponent, respectively. Note that the drug mechanism function (eq. 14 , upper two rows) lacks the constant term (1) typically found in these functions (eq. 14, bottom two rows) when no baseline information is available. This is because the locomotor activity is zero or negligible in the absence of drug in this experiment.

The drug mechanism function is then incorporated into the systems equation describing the pharmacological response (eq. 15):

$$
\frac{d R}{d t}=S\left(A_{\mathrm{b}}\right)-k_{\text {out }}(R) \cdot R
$$

where $k_{\text {in }}$ has been removed since the baseline value of locomotor activity is zero. In light of the linear decay of response (Fig. 8), the loss of response will be modeled as a saturable term $k_{\text {out }}(R)$ with a fractional turnover rate $k_{\text {out }}$ as a function of $R$ (eq. 16):

$$
k_{\text {out }}(R)=k_{\text {out,max }} \cdot \frac{1}{k_{\mathrm{M}}+R}
$$

The terminal slope of the response-time course when $R>>k_{\mathrm{M}}$ is then written as shown in eq. 17:

$$
\frac{d R}{d t}=-k_{\text {out,max }} \approx 30 \mathrm{U} / \min
$$

where $k_{\text {out,max }}$ is approximately 30 response units per minute. Similarly, the slope of the rise in response equals the following (eq. 18):

$$
\frac{d R}{d t}=S_{\max }-k_{\text {out,max }} \approx 90 \mathrm{U} / \mathrm{min}
$$

where $S_{\max }$ can be approximated as $90+30=$ $120 \mathrm{U} / \mathrm{min}$, provided both processes are saturated. The pharmacological response will reach an equilibrium state if the rate of production is less than the maximal rate of loss; that is, when the first term in eq. 15 is smaller than the maximal upper bound of the second term [i.e., if $S\left(A_{\mathrm{b}}\right)<k_{\text {out,max }}$ ]. The equilibrium biophase amount versus response relationship becomes the following (eq. 19):

$$
R_{\mathrm{SS}}\left(A_{\mathrm{b}}\right)=k_{\mathrm{M}} \cdot \frac{S\left(A_{\mathrm{b}}\right)}{k_{\text {out,max }}-S\left(A_{\mathrm{b}}\right)}
$$

The data are rich since they contain high-resolution response-time courses at two dose levels. It appears that the dexamphetamine-locomotor activity scores are a 
TABLE 4

Final parameter estimates of first-order and bolus models, their relative S.D. (CV\%), and their objective function value (WSSR)

\begin{tabular}{lccccr}
\hline \multirow{2}{*}{ Parameter } & \multicolumn{2}{c}{$\begin{array}{c}\text { First-Order } \\
\text { Biophase Model }\end{array}$} & & \multicolumn{2}{c}{$\begin{array}{c}\text { Bolus Biophase } \\
\text { Model }\end{array}$} \\
\cline { 2 - 3 } & Estimate & CV\% & & Estimate & CV\% \\
\hline$S_{\max }$ & 249 & 1 & & 190 & 7 \\
$K^{\prime}\left(\right.$ first-order) $\left(\mathrm{h}^{-1}\right) K$ (bolus) $\left(\mathrm{h}^{-1}\right)$ & 5.96 & 4 & & 2.54 & 6 \\
$\mathrm{SD}_{50}(\mu \mathrm{g})$ & 1.02 & 4 & & 1.43 & 8 \\
$n_{\mathrm{H}}$ & 1.63 & 5 & & 2.05 & 18 \\
$k_{\text {out, max }}\left(\mathrm{h}^{-1}\right)$ & 30.1 & 4 & & 32.9 & 4 \\
$k_{\mathrm{M}}\left(\mathrm{h}^{-1}\right)$ & 0.001 & 9 & & 0.001 & 1 \\
$\mathrm{WSSR}$ & 768 & & & 640 & \\
\hline
\end{tabular}

WSSR, weighted sum of squared residuals.

linear relation of the time postpeak independent of dose. Equation 16 is therefore suggested as a reasonable approximation of the zero-order decline of responsetime data. The model is simultaneously fit to both response-time courses only changing the dose between the two datasets. Dose-normalized areas increased with dose, which indicates some kind of saturation in the loss of response and/or saturable stimulation function such as eq. 16. This is also supported by the peak shift in the response-time courses.

3. Results and Conclusions with Respect to DoseResponse-Time Analysis. The regression lines of the two biophase models (eq. 13) coupled to the pharmacological model (eqs. 14 and 15) of the data are shown in Fig. 8. The final parameter estimates are listed in Table 4, together with their precision [coefficient of variation $(\mathrm{CV} \%)]$.

4. Pharmacodynamic Interpretation and Comments. Normally, locomotor activity in rodents is dependent on several factors, including novelty of the environment versus habituation and phase of the day-light cycle (rodents being nocturnal). In the locomotor stimulation case described above, the rats are likely to have been preadapted to the mobility meter boxes in the light phase of the 24-hour cycle to minimize exploratory activity. From the modeling perspective, this simplifies analysis of the data because baseline may be set to zero, both prior to and after the period of drug challenge. The turnover rate $k_{\text {in }}$ was therefore eliminated from eq. 15, and the drug mechanism function could be condensed. A clear peak shift (time shift in $t_{\max }$ values) was observed in response-time data with increasing doses of dexamphetamine. The linear decay of the locomotor response toward baseline may be contingent on the elimination of dexamphetamine from the biophase, but it may additionally involve desensitization of central dopamine neuronal and/or receptor mechanisms. It is clear from the simulated predicted plasma concentration-time courses (superimposed solid gray lines on experimental and model-predicted response-time courses in Fig. 8) that a biophase compartment is needed to capture timecourse differences between plasma exposure and locomotor activity after intraperitoneal dosing. Evidently,
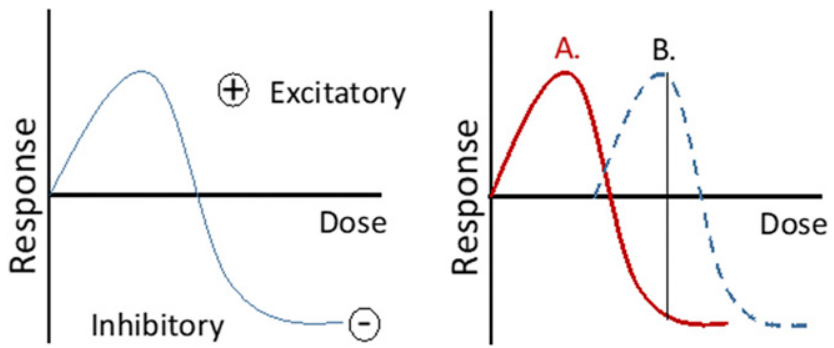

Fig. 9. (Left) Schematic illustration of the principles of a biphasic doseresponse relationship. (Right) Example showing the complexity of comparing two compounds with a slight shift between their dose-response relationships. Even though both have similar shapes, one compound may demonstrate an excitatory (stimulant) response at the same dose as the other compound demonstrates an inhibitory (depressant) response. In this particular situation, DRT analysis may fail to characterize their pharmacodynamic properties. Exposure-response analysis would therefore be feasible option and is a better alternative.

plasma concentrations do not directly drive the pharmacological response, and access to brain target levels aligned to the plasma time course of dexamphetamine would have been a useful addition to the locomotor activity data to verify the biophase structure. Note that the model-predicted $k_{\mathrm{M}}$ value is very low $(0.001$ response units), which therefore makes the decline of locomotor activity a zero-order process at the present dose levels, whereas the plasma concentrations follow a first-order disappearance with a drug half-life of about 90 minutes. Information about the shape of the drug time course in the biophase in relation to plasma and locomotor activity levels could therefore potentially give clues to underlying contributory mechanisms and events. Although it is beyond the scope of this review, it would also be interesting to see what further insights on pharmacodynamic similarities and differences of drugs able to stimulate motor activity (e.g., amphetamine, cocaine, ethanol, caffeine, etc.) might be gained from a crosscomparison DRT analysis based on multiple dose levels, high-resolution behavioral responses, and corresponding plasma (or, ideally, biophase) data of such agents. This said, it should be recalled that several of the aforementioned agents possess dose- (concentration-) and time-dependent psychostimulant as well as depressant properties and therefore may be challenging to compare or rank by means of a DRT approach (see Fig. 9). Drugs with well known biphasic exposureresponse relationships include ethanol, caffeine, and morphine (see Dafters and Taggart, 1992; Calabrese, 2008), where the extent of excitatory and depressant actions depend on the drug in question, dose, and time after administration. DRT analysis may also be of limited value for the assessment of safety margins, where establishing systemic exposure to unbound drug concentrations should instead be the method of choice (Gabrielsson and Hjorth, 2012, 2018; Gabrielsson et al., 2018). 

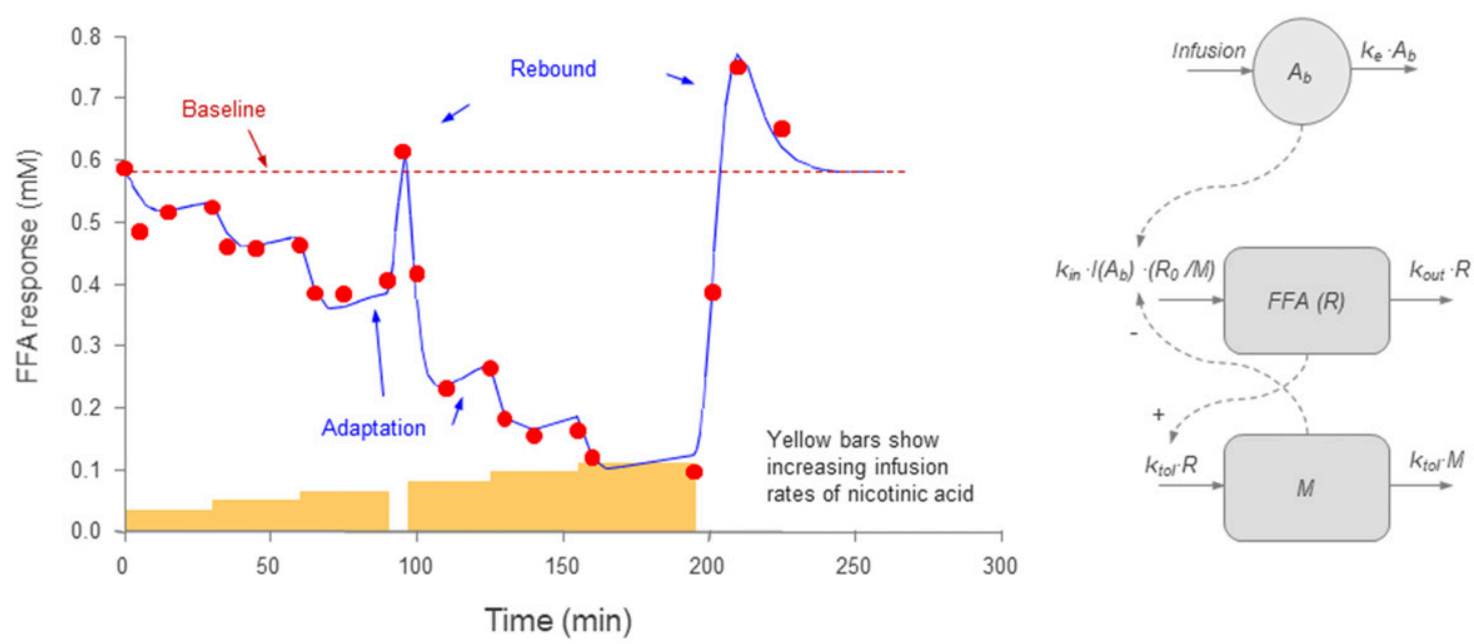

Time (min)

Fig. 10. (Left) Observed (filled symbols) and model-predicted (solid line) response vs. time data after a multiple intravenous infusion regimen of NiAc, followed by washout (Isaksson et al., 2009). The infusion pump stopped for a brief period at about 90 minutes until the next syringe had been loaded. During this event, there is a rapid return of response toward and past the baseline. The six yellow bars represent six different infusion rates $(0.008$, $0.016,0.032,0.064,0.128$, and $0.256 \mathrm{U} / \mathrm{min}$ ). (Right) Schematic illustration of the proposed feedback model. Solid lines denote flows and dashed lines denote control steps. $A_{\mathrm{b}}$ and $k_{\mathrm{e}}$ denote the biophase amount and elimination rate constant out of the biophase, respectively. $k_{\text {in }}$ denotes the turnover rate, $I\left(A_{\mathrm{b}}\right)$ is the drug mechanism function, $R_{0}$ is the baseline response, $M$ is the moderator compartment, and $k_{\text {out }}$ and $k_{\text {tol }}$ are the fractional turnover rate and fractional turnover rate of the moderator compartment, respectively. FFA is the free fatty acid response biomarker $R$. The moderator compartment $M$ represents all lumped feedback mechanisms in fatty acid regulation (e.g., insulin).

\section{Case Study 3: Dose-Response-Time Analysis of Functional Adaptation}

1. Background. This case study is aimed at demonstrating DRT analysis containing functional adaptation (Isaksson et al., 2009) of a metabolic response to drug (NiAc) treatment. The biophase kinetics, $k_{\mathrm{e}}$, will be estimated simultaneously with system parameters $\left(k_{\text {out }}\right.$ and $\left.k_{\text {tol }}\right)$ and drug parameters $\left(\mathrm{ID}_{50}\right.$ and $n$ ). The mechanism of action is via inhibition of the turnover rate $\left[I\left(A_{\mathrm{b}}\right) \cdot k_{\text {in }}\right]$. Full inhibition of response is assumed with a sufficiently high dose. Data are obtained after six consecutive intravenous infusions of NiAc (plasma half-life of 1 to 2 minutes) followed by washout (Fig. 10). The plasma concentration-time course of NiAc will therefore have the shape of a square wave due to the short half-life.

The proposed model is shown in Fig. 10 (right). The model contains two parallel turnover models connected via $k_{\mathrm{tol}} \cdot R$ and $k_{\text {in }} \cdot R_{0} / M$ serving as input to the moderator compartment $(M)$ and $R$, respectively. The ratio of $k_{\text {tol }}$ to $k_{\text {out }}$, also called $\kappa$, gives an idea about the speed of tolerance development and the possibility of observing rebound. This system is shown to be applicable to many diverse situations with drug tolerance and rebound (Gabrielsson and Peletier, 2008).

2. Models, Equations, and Exploratory Analysis. The multiple zero-order input steps and first-order loss of the biophase compartment are expressed as a differential equation (eq. 20):

$$
\frac{d A_{\mathrm{b}}}{d t}=\text { Input rate }-K \cdot A_{\mathrm{b}}
$$

Input rate, $K$, and $A_{\mathrm{b}}$ are the escalating zero-order infusion rates of $\mathrm{NiAc}$, the elimination rate constant, and the biophase amount, respectively. The biophase function is then driving the drug mechanism function via an inhibitory process $\left(I_{\max }, \mathrm{ID}_{50}\right.$, and $\left.n_{\mathrm{H}}\right)$, where the $I_{\text {max }}$ parameter is set to a constant value of 0.95 (eq. 21 ).

$$
I\left(A_{\mathrm{b}}\right)=1-\frac{I_{\max } \cdot A_{\mathrm{b}}^{n_{\mathrm{H}}}}{\mathrm{ID}_{50}^{n_{\mathrm{H}}}+A_{\mathrm{b}}^{n_{\mathrm{H}}}}
$$

The biophase and drug mechanism functions are then incorporated into one of the turnover systems of a response model slightly different from the one presented in Isaksson et al. (2009), as shown in eq. 22:

$$
\frac{d R}{d t}=k_{\text {in }} \cdot I\left(A_{\mathrm{b}}\right) \cdot \frac{R_{0}}{M}-k_{\text {out }} \cdot R
$$

where $k_{\text {in }}$ is the turnover rate, $M$ is the moderator compartment, $k_{\text {out }}$ is the fractional turnover rate, and $I(A)$ is the drug inhibitory function. The action of $M$ on $R$ was assumed to occur via inhibition of production of $R$, rather than via stimulation of loss of $R$, as the former is more attractive from both a mechanistic and an energy-conserving point of view. The $R_{0} / M$ ratio denotes the baseline-normalized feedback of the moderator $M$. The turnover of the moderator $M$ is shown in eq. 23:

$$
\frac{d M}{d t}=k_{\mathrm{tol}} \cdot R-k_{\mathrm{tol}} \cdot M
$$

$I_{\max }$ is assumed to be 1 , which means $100 \%$ inhibition for a very high dose. Input is then governed by the multiple consecutive intravenous infusion regimens. 
TABLE 5

Initial and final model parameter estimates and their corresponding precision $(\mathrm{CV} \%)$

\begin{tabular}{lccr}
\hline Parameter & Initial Estimate & Final Estimate & CV\% \\
\hline$R_{0}(\mathrm{mM})$ & 0.6 & 0.58 & 3 \\
$k_{\text {out }}\left(\min ^{-1}\right)$ & 0.1 & 0.59 & 36 \\
$k_{\text {tol }}\left(\min ^{-1}\right)$ & 0.07 & 0.058 & 25 \\
$n_{\mathrm{H}}$ & 1 & 1.40 & 7 \\
$\mathrm{ID}_{50}\left(\mathrm{mmol}^{-1}\right)$ & 0.1 & 0.13 & 26 \\
$K\left(\min ^{-1}\right)$ & 0.2 & 0.22 & 13 \\
\hline
\end{tabular}

The model parameters of eqs. $20-23$ are the baseline value $R_{0}$, the fractional turnover rate of response $k_{\text {out }}$, the rate constant of tolerance development $k_{\mathrm{tol}}$, and the biophase kinetic rate constant $k_{\mathrm{e}}$. $\mathrm{ID}_{50}$ is the amount in the system resulting in $50 \%$ of $R_{\max }$. The $n$ parameter allows more flexibility in the inhibitory function. The baseline parameter $R_{0}$ can be approximated to 0.6 units.

The $k_{\text {tol }}$ parameter governs the rebound between 200 minutes (at the end of infusion) and 240 minutes (return of response to baseline). If we assume that four half-lives of $k_{\mathrm{tol}}\left(t_{1 / 2, k \mathrm{tol}}\right)$ have elapsed during the period from 240 to 200 minutes (i.e., 40 minutes), then the halflife is about 10 minutes. The first-order $k_{\text {tol }}$ parameter then becomes $\ln (2) / 10$, which is about $0.07 \mathrm{~min}^{-1}$.

The overall goal is to explain the complex behavior of the biomarker $R$ as a simple biophase amount-response relationship. At pharmacodynamic steady state and with inhibition of $k_{\text {out }}$, the response becomes the following (eq. 24):

$$
R_{\mathrm{SS}}=\left\{\begin{array}{c}
R_{0} \cdot I\left(A_{\mathrm{b}, \mathrm{SS}}\right) \\
R_{0} \cdot \sqrt{I\left(A_{\mathrm{b}, \mathrm{SS}}\right)}
\end{array}\right.
$$

3. Results and Conclusions with Respect to DoseResponse-Time Analysis. High consistency was found between the observed and model-predicted data (Fig. 10, left). Table 5 contains initial and final parameter estimates and their corresponding CV\% values. Note that $k_{\text {tol }}\left(0.058 \mathrm{~min}^{-1}\right)$ is 10 times smaller than $k_{\text {out }}$ $\left(0.59 \mathrm{~min}^{-1}\right)$. The $\kappa$ parameter is defined as $k_{\text {tol }} / k_{\text {out }}$, which suggests that it takes 10 times longer to develop the tolerance equilibrium than the response equilibrium. The size of $\kappa(<1)$ also suggests a substantial rebound effect, provided that the kinetics of the test compound does not confound the rebound development.

Figure 11 shows two simulations of eq. 24, representing a tolerant and nontolerant system, respectively, at equilibrium. We have by means of a modeling approach (eqs. 20-23; Fig. 10) condensed the complexity into a relatively simple dose-response relationship, displayed in Fig. 11. A tolerant system requires more drug to give the same response at equilibrium. In this case example, the tolerant system also shows a reduced ability to suppress fatty acids to the same low level $(0.05 \mathrm{mM})$ as seen in the nontolerant condition. This is revealed by the upward shift of the red dose-response curve.

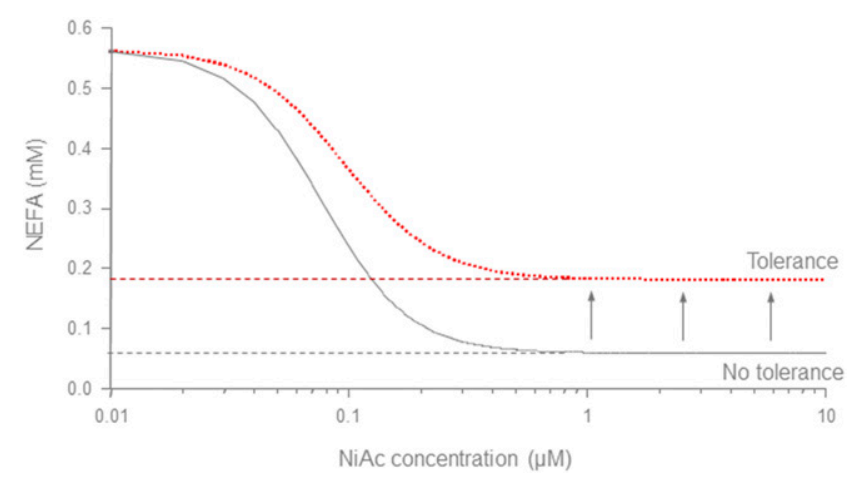

Fig. 11. Response vs. NiAc concentration $\left(A_{\mathrm{b}, \mathrm{ss}} / V_{\mathrm{ss}}\right)$ relationship at equilibrium. Curves are normalized to the same baseline value. The gray curve shows a system without feedback, and the red curve shows a system with feedback. The same dose results in less effect in a tolerant system at equilibrium as compared to a nontolerant system.

Data showed a baseline value prior to test compound administration. An almost complete inhibition of response was seen with the highest dose, which suggests that more extensive blockage of response is possible in a nontolerant system. Data also demonstrated adaptation with rebound and a limited ability to suppress the level of free fatty acids (FFAs) in this condition (Fig. 11).

4. Pharmacodynamic Interpretation and Comment. Adaptational phenomena in physiology and pharmacology are commonplace and need to be accounted for in all drug treatment contexts involving repeated dosing (chronic indications). Needless to say, the degree of adaptational and compensatory alterations varies broadly across physiologic response, disease, target, and drug class conditions. In the case example described above, the nontolerant system responded to NiAc with a marked shutdown of the FFA response. That said, it should be noted that FFA concentrations were not totally suppressed; this suggests that at least for the type of rapid-acting drug target intervention represented by NiAc, other physiologic mechanisms will sustain a minimum FFA concentration limit. In turn, the upward shift of this minimum seen in the tolerant system may indicate adjustment in the sensitivity of the primary NiAc target sites in its intended biophase and/or recruitment of other buffering/compensatory mechanisms to ascertain upholding of "safe" FFA levels.

\section{Case Study 4: Dose-Response-Time Analysis of Antipsychotic Effects (Brief Psychiatric Rating Scale Scores)}

1. Background. This case study highlights the assessment of the antipsychotic action of remoxipride in patients with schizophrenia (Lewander et al., 1990). The antipsychotic efficacy of the dopamine receptorblocking agent remoxipride was established in a series of clinical studies, and analysis suggested three important aspects of its effects in acute-phase schizophrenia. First, 25\%-30\% of patients did not respond. Second, the remoxipride treatment response appeared to affect both 

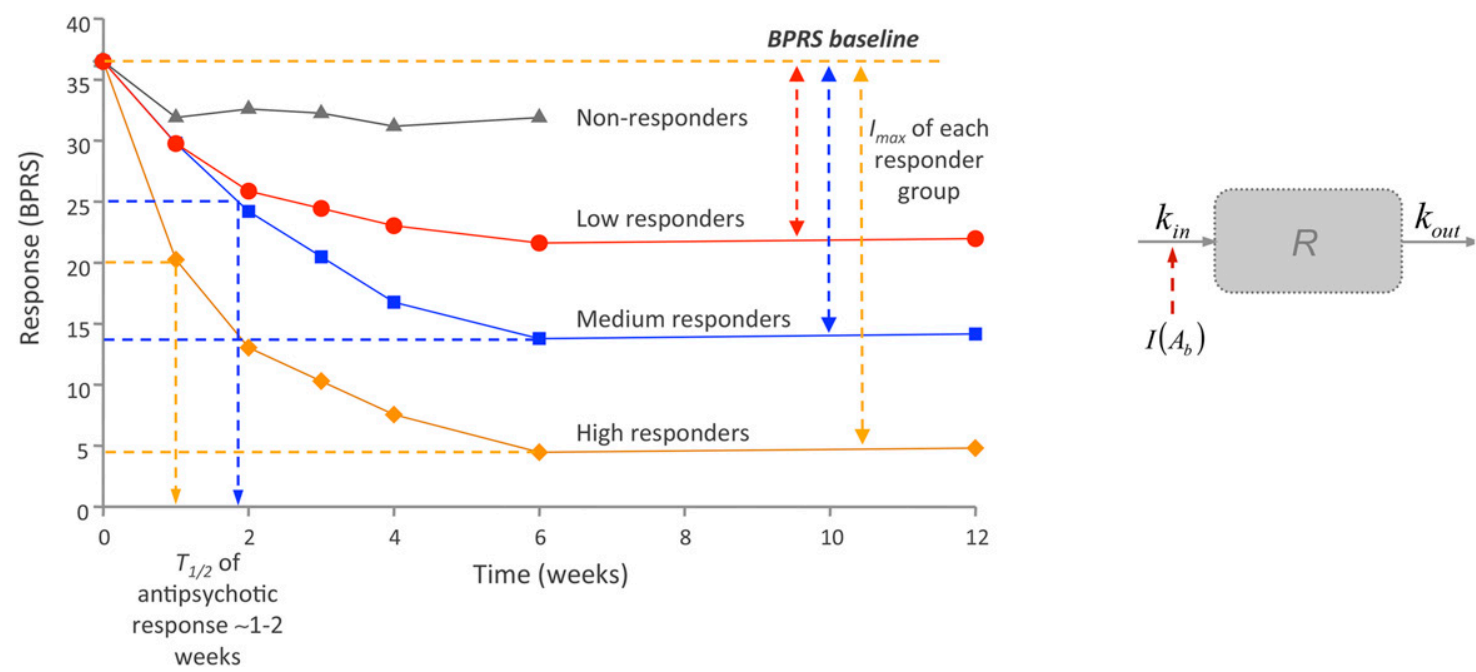

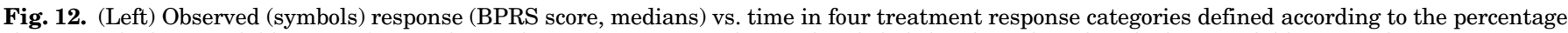

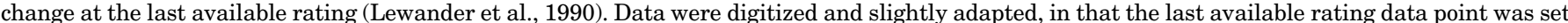

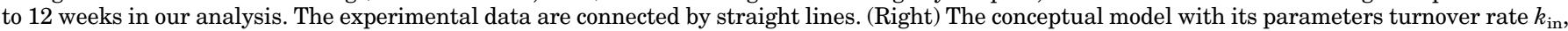
fractional turnover rate $k_{\text {out }}$, and drug mechanism function $I\left(A_{\mathrm{b}}\right)$. R denotes the BPRS score.

positive and negative symptoms of the disorder to a similar degree. Third, the onset of antipsychotic action in responders seemed to be unusually rapid (i.e., within 1 to 2 weeks). Patients with schizophrenia in an acute phase of the illness received remoxipride (dose range, 120-600 mg/d) and were repeatedly scored for symptom severity across a time period of at least 6 weeks. The observed mean BPRS time courses in four treatment response categories are shown in Fig. 12.

The patients were divided into four categories. Nonresponders were defined as having a $<25 \%$ reduction, low responders as a $25 \%-49 \%$ reduction, medium responders as a 50\%-74\% reduction, and high responders as a $>75 \%$ reduction in total BPRS scores from baseline to the last available rating.

The turnover model of the antipsychotic responsetime data is given in eq. 25 . This is therefore an example of evaluating the effect of a drug via usage of a disease model, with a decrease in BPRS score being indicative of a reduction in disease severity. Drug exposure and responder category are embedded in the $I_{\max }$ parameter for each responder category.

2. Models, Equations, and Exploratory Analysis. Exposure to remoxipride was assumed to be constant across the whole period of BPRS score measurements. The half-life of remoxipride is about 4 to 5 hours in plasma, compared with the half-life of BPRS score (in weeks). The analysis focused on modeling BPRS responder category (Fig. 12) as a function of time. The actual mean doses of the drug used in the studies in the last 4- to 6-week period ranged from 226 to $556 \mathrm{mg} /$ day (typically approximately $400 \mathrm{mg} /$ day; Lewander et al., 1990), but "dose" was not used as a covariate for separating the responder categories.

The rate of change of response $d R / d t$ (Fig. 12, right) can be described by eq. 25 :

$$
\left\{\begin{array}{l}
\frac{d R}{d t}=k_{\text {in }} \cdot I\left(A_{\mathrm{b}}\right)-K_{\text {out }} \cdot R \\
\frac{d R}{d t}=k_{\text {out }} \cdot\left(R_{0} \cdot I\left(A_{\mathrm{b}}\right)-R\right)
\end{array}\right.
$$

where $k_{\text {in }}, k_{\text {out }}, R_{0}$, and $I\left(A_{\mathrm{b}}\right)$ are the turnover rate, fractional turnover rate, baseline biophase response, and inhibitory drug mechanism function (eq. 26), respectively. Equation 25 will, under constant drug exposure, describe the drug action in Fig. 12. The time to dynamic steady state, assuming a constant biophase exposure to remoxipride, $A_{\mathrm{bss}}$, will be governed by $k_{\text {out }}$. It is reasonable to assume that the exposure can be held constant across two consecutive dosing intervals. Evidently, the time course of the response (half-life of 1 to 2 weeks) is not directly correlated to the plasma half-life of the drug, which is much shorter (4 to 5 hours). It was therefore decided to model the inhibitory effect of drug according to the following expression:

$$
I\left(A_{\mathrm{b}}\right)=1-I_{\max }
$$

where $I_{\max }$ is the rating category of responders (nonresponders and low, medium, and high responders). In the absence of drug, $I\left(A_{\mathrm{b}}\right)=1$ and the baseline level of response becomes (eq. 27)

$$
R_{0}=\frac{k_{\text {in }}}{k_{\text {out }}}
$$

In the presence of drug, the level of response becomes (eq. 28)

$$
R_{\mathrm{SS}}=\frac{k_{\text {in }}}{k_{\text {out }}} \cdot\left(1-I_{\max }\right)=R_{0} \cdot\left(1-I_{\max }\right)
$$

where $R_{\mathrm{ss}}$ is the minimum steady-state response. The lower the value of $R_{\mathrm{ss}}$, the better the drug effect. If we 


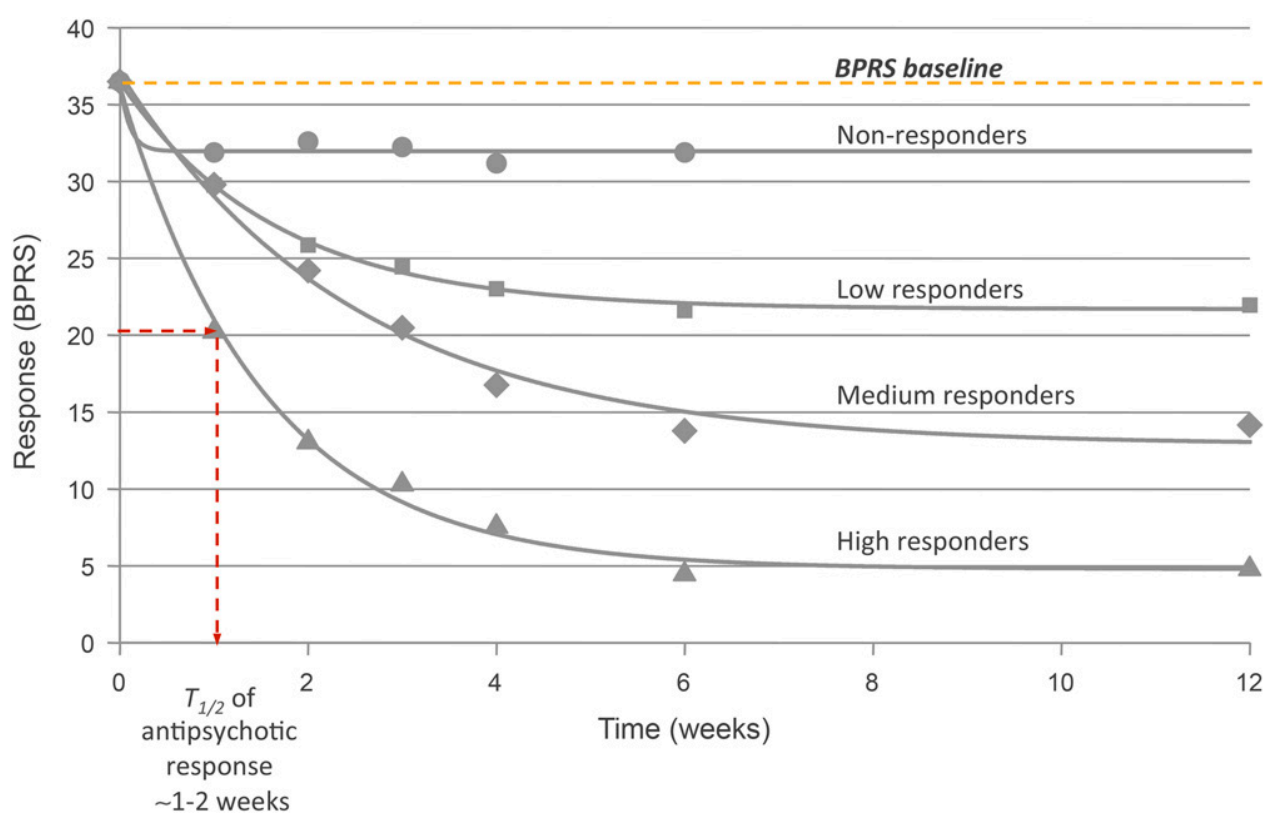

Fig. 13. Observed (symbols) and model-predicted (lines) response (BPRS score) vs. time in four treatment response categories defined according to the percentage change at the last available rating (Lewander et al., 1990). Dotted lines represent the dynamic half-life (BPRS scores) in the $>75 \%$ responder category: approximately 1 week.

had full response in that $I_{\max }$ equaled unity (1), then $R_{\mathrm{ss}}$ would equal 0 . The difference between $R_{\mathrm{ss}}\left(R_{\min }\right)$ and $R_{0}$ is given by eq. 29 .

$$
\left\{\begin{array}{c}
\Delta R=R_{0}-R_{\mathrm{SS}}=\frac{k_{\text {in }}}{k_{\text {out }}}-\frac{k_{\text {in }}}{k_{\text {out }}} \cdot\left(1-I_{\max }\right)= \\
\frac{k_{\text {in }}}{k_{\text {out }}} \cdot I_{\max }=R_{0} \cdot I_{\max }
\end{array}\right.
$$

From the intercept of the response-time curve with the effect axis (Fig. 12), the baseline value $R_{0}$ (36.5 units) can be obtained. $R_{0}$ is the ratio of $k_{\text {in }} / k_{\text {out }}$, if we assume eq. 27 to be an appropriate description of the drug-free state. The $k_{\text {out }}$ parameter is obtained from the log-linear slope of the downswing of the highest responder $(>75 \%)$ curve (eq. 30):

$$
K_{\text {out }}=\frac{\ln (36.5)-\ln (10.3)}{0-3} \approx 0.42 \mathrm{wk}^{-1}
$$

The $k_{\text {in }}$ parameter can then be calculated ( $\left.15.4 \mathrm{U} / \mathrm{wk}\right)$ from the baseline value ( 36.5 units) and $k_{\text {out }}\left(0.42 \mathrm{wk}^{-1}\right)$. $I_{\max }$ was estimated according to eq. 26 to be 0.8 for the highest responder $(>75 \%)$ group. That value was used as the starting value for $I_{\max }$ in all groups.
3. Results and Conclusions with Respect to DoseResponse-Time Analysis. Figure 13 displays observed data together with model-predicted curves of the turnover model. Table 6 gives the final parameter estimates of the four patient categories together with the parameter precision. Note that the parameter precision was high in general, except for the nonresponder category. The half-life of response ranged between 1.1 weeks $(25 \%-49 \%$ and $>75 \%$ responder categories) and 1.7 weeks $(50 \%-75 \%$ responder category). The $I_{\max }$ values were $0.41,0.65$, and 0.87 for the $25 \%-49 \%, 50 \%-74 \%$, and $>75 \%$ responder categories, respectively (Table 6 ).

The quantitative category-response-time analysis approach enabled us to assess the actual responder values $\left(I_{\max }\right)$ as well as the half-life of response for each category. Since the half-life of response was about a week, we know that $90 \%$ response should have been established within 3 to 4 weeks (i.e., three to four half-lives). The half-life of response $\left[\ln (2) / k_{\text {out }}\right]$ clearly demonstrates the adjustment of the dopaminergic system to drug intervention. It follows in this case that whether a patient responds to the remoxipride therapy may be possible to establish within 1 to 2 weeks from

TABLE 6

Final parameter estimates of the four patient categories

\begin{tabular}{|c|c|c|c|c|}
\hline \multirow{2}{*}{ Parameter } & \multirow{2}{*}{$\begin{array}{l}\text { Nonresponders } \\
(<25 \% \text { Reduction })\end{array}$} & \multicolumn{3}{|c|}{ Responders } \\
\hline & & $25 \%-49 \%$ Reduction & $50 \%-74 \%$ Reduction & $>75 \%$ Reduction \\
\hline$I_{\max }$ & $0.13 \pm 19$ & $0.41 \pm 2$ & $0.65 \pm 4$ & $0.87 \pm 2$ \\
\hline$k_{\text {in }}($ scores $/ \mathrm{wk})$ & $45 \pm 60$ & $22 \pm 7$ & $15 \pm 20$ & $24 \pm 9$ \\
\hline
\end{tabular}

Data are presented as the estimate $\pm \mathrm{CV} \%$. 
the start of drug treatment. Therefore, the modeling lesson to be learned from this case study is that frequent (two or three times daily) dosing may not necessarily be needed for a response such as an antipsychotic effect when the half-life of the response is much greater than the plasma half-life of the drug, unless there are safety issues related to drug intake. The time to dynamic steady state is unaffected by dose and/or dose frequency. In other words, pharmacokinetic reasoning cannot stand alone but has to be integrated with dynamics in the decision-making process.

4. Pharmacodynamic Interpretation and Comments. Ideally, we would have liked to see a return to baseline after the cessation of drug treatment, as this may have given an indication of possible rebound or adaptation. Although withdrawal of a drug and therefore reduction (and possibly obliteration) of the response may be information rich in terms of determining the potency of the drug $\left(\mathrm{EC}_{50} / \mathrm{IC}_{50}\right)$, drug-free periods may jeopardize patient health and should therefore be avoided (certainly in difficult psychiatric afflictions). Although such a design may thus be considered when developing a new drug for schizophrenia, its application is complicated by the typical chronic and progressing nature of this illness, along with the fact that a significant number of patients may have developed supersensitive dopamine (and possibly other) target mechanisms as a sequel to having been previously exposed long term to one or more antipsychotic drugs. Careful selection of the de novo patients may be envisaged as a potential means to circumvent said complications, and thereby gather more complete DRT (coupled to plasma drug exposure data) information from clinical studies to the benefit of future antipsychotic drug development. From a strictly therapeutic point of view, the data also underline the marked heterogeneity encountered among patients diagnosed with schizophrenia. Identification of underlying reasons for this may pave the way for better, more individualized treatments. Incidentally, the analysis of this case study suggests the intriguing possibility that it might be possible to identify responders and nonresponders already in the treatment initiation phase. Further attesting to this prospect, comparable data were found with haloperidol in the same study (Lewander et al., 1990) as well as with other agents in a number of other literature reports (e.g., Levine and Leucht, 2010; Marques et al., 2011; Stauffer et al., 2011), all of which display very similar patterns with respect to antipsychotic drug treatment onset and response trajectories in patient subgroups. DRT modeling across these studies indicates that responders (including "high," "medium," and "low" responders) display a half-life of 1 to 2 weeks for symptom improvement and 4-6 weeks to pharmacodynamic steady state, and it also suggests early spotting of patients with poor or no response to (antidopaminergic) treatment. That said, any comparisons of efficacies, potencies, and/or safety-related issues between compounds, species, or studies must be based on plasma exposure rather than dose, as pharmacokinetic properties such as bioavailability, clearance, and half-life also need to be factored in. Further stratified DRT analyses of responses to other psychiatric drug treatment classes would be of great interest toward future individualized pharmacotherapy approaches and strategies.

\section{E. Case Study 5: Dose-Response-Time Analysis of Bacterial Count}

1. Background. This case study highlights some complexities in DRT modeling of bacterial growth/kill data. Antibacterial compound $\mathrm{X}$ was being developed (Gabrielsson and Weiner, 2010). To establish its potency in a resistant bacterial strain, a 10,000-U dose of bacteria was injected into the bloodstream of five groups of Wistar rats. A dose of 0 (vehicle control), 1.5, 2, 4, or $6 \mu \mathrm{g}$ of the antibiotic was given to each of the groups. Blood was drawn at selected time points for bacterial count (Fig. 14).

2. Models, Equations, and Exploratory Analysis. The bacterial growth/kill model is shown in Fig. 14 (right). The vehicle dose was included to explore the natural bacterial growth, $k_{\mathrm{g}}$, in the absence of antibiotic. The model was extended to incorporate an upper growth-limiting factor, $1-N / N_{\max }$ (eq. 31):

$$
\frac{d N}{d t}=k_{\mathrm{g}} \cdot N \cdot\left(1-\frac{N}{N_{\max }}\right)-k_{\mathrm{k}} \cdot f\left(A_{\mathrm{b}}\right) \cdot N
$$

where $N_{\max }$ is the maximum value of the bacterial count in the system (defined by the bacterial growth endpoint in the vehicle-treated group). When the number of bacteria $N$ approaches the steady-state level $N_{\max }$, the factor $1-N / N_{\max }$ approaches zero and bacterial growth is temporarily stopped. During the early cell kill phase, the decline in bacterial count becomes

$$
\frac{d N}{d t}=-k_{\mathrm{k}} \cdot f\left(A_{\mathrm{b}}\right) \cdot N
$$

Figure 15 shows the results from fitting the extended growth/kill model to the bacterial count versus time dataset. Note that the starting values at time 0 were not equal for the different groups of animals (see Table 7, $\left.N_{01-05}\right)$. From the growth of bacteria in the vehicle group, the steady-state value of growth/kill can be estimated to about $25-30,000$ bacteria.

3. Results and Conclusions with Respect to DoseResponse-Time Analysis. The proposed DRT model nicely fits the five $(0,1.5,2,4$, and $6 \mu \mathrm{g})$ colonyforming unit time courses in Fig. 14, and Table 7 shows the final parameter estimates and their CV\%. DRT analysis lent itself to assessment of the growth and kill parameters. This model may now be used for optimizing 

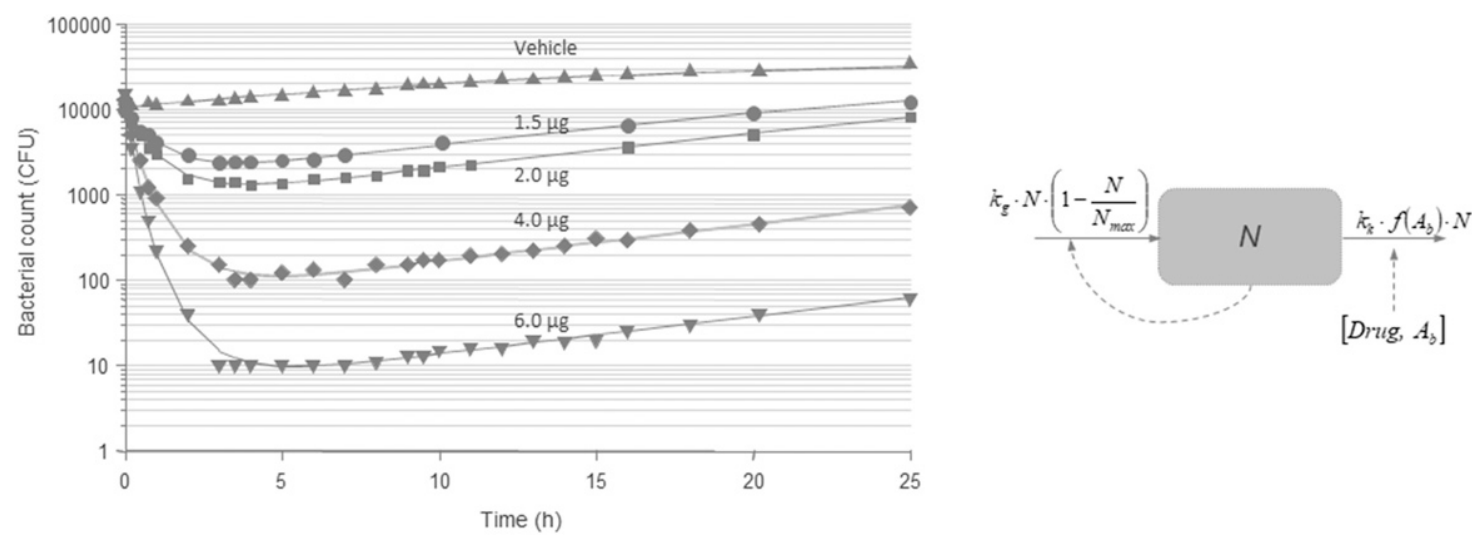

Fig. 14. (Left) Observed (symbols) and predicted (lines) response (bacterial count, CFU) vs. time after administration of $1.5,2,4$, and $6 \mu \mathrm{g}$ of a new antibiotic. Note the large range of counts (more than three orders of magnitude). (Right) First-order bacterial growth coupled to the second-order bacterial kill model. The first-order growth $k_{\mathrm{g}} \cdot N \cdot\left(1-N / N_{\max }\right)$ is saturable by means of the $\left(1-N / N_{\max }\right)$ term, where $k_{g}, N$, and $N_{\max }$ denote the first-order growth rate constant, the number of bacteria, and the maximum bacterial count, respectively. The bacterial kill process is approximated by $-k_{\mathrm{k}} f\left(A_{\mathrm{b}}\right) \cdot N$, where $k_{\mathrm{k}}, N$, and $A_{\mathrm{b}}$ denote the first-order kill rate constant, the number of bacteria, and the plasma concentration of drug. CFU, colony-forming unit.

the next study on repeated dosing with an alternative dosing regimen.

4. Pharmacodynamic Interpretation and Comments. Bacterial antibiotic resistance development is a well known, global problem in need of novel drugs and solutions. Analysis of the DRT data in the study described in this case example gave robust parameter estimates to assess growth and kill rates upon exposure to the antibacterial agent X. The data in Fig. 14 suggest that bacterial (re)growth was kept at bay for approximately 6 hours after administration of the drug, after which growth exceeded the kill rate. Thus, from a treatment perspective, it could be suggested that a second dose of antibiotic given within the 6-hour time frame should be considered to more effectively eradicate the infection. The data derived from the DRT model and analysis therefore had significant impact on the onward design of drug treatment protocols, and potentially also for the optimization of pharmaceutical formulations for therapeutic use.

\section{F. Case Study 6: Dose-Response-Time Analysis of Cortisol-Adrenocorticotropic Hormone Action}

1. Background. Data were scanned from an article by Urquhart and Li (1968), who studied the dynamics of cortisol secretion of the perfused canine adrenal gland in situ upon stimulation by ACTH. The dogs were acutely hypophysectomized to eliminate endogenous ACTH. The cortisol secretion rate (response) was expressed as the product of the venous blood flow and the concentration of cortisol in adrenal venous blood (Fig. 16). The proposed model captures feedback regulation at constant drug exposure. This dataset therefore violates the previous assumption about time invariant systems.

2. Models, Equations, and Exploratory Analysis. The proposed feedback model is shown in Fig. 16 (right). Let us assume that the rise and fall of the cortisol response $R$ shown in Fig. 16 (left) can be modeled by the following simple feedback model (eq. 33):

$$
\frac{d R}{d t}=S\left(A_{\mathrm{b}}\right) \cdot k_{\text {in }}-k_{\text {out }} \cdot M
$$

$S\left(A_{\mathrm{b}}\right)$, which represents the ACTH drug mechanism function, stimulates the production of $R$, which is then counterbalanced by means of the endogenous modulator that we denote as $M$. Note that the loss of $R$ is indirectly governed by means of $M . M$ is in turn governed by $R$ and the rate constant for development of tolerance $k_{\text {tol }}$, which can be written as shown in eq. 34 :

$$
\frac{d M}{d t}=k_{\mathrm{tol}} \cdot R-k_{\mathrm{tol}} \cdot M
$$

The $k_{\text {tol }}$ parameter was selected to govern both production and elimination of $M$ in this particular example. However, this may not always be the case where data contain more information about the different rate processes. When $R$ increases, the production of modulator $M$ is stimulated and then increases. The increase in $M$ counterbalances $R$ by increasing the rate of loss of $R$. It is assumed that the rates in and out of $M$ are governed by the first-order rate constant for tolerance $k_{\text {tol. }}$.

The ACTH drug mechanism function $S\left(A_{\mathrm{b}}\right)$ is written as follows (eq. 35):

$$
S\left(A_{\mathrm{b}}\right)=\left[A_{\mathrm{b}}\right]^{n}
$$

where the ACTH concentration is either $1 \mu \mathrm{U} / \mathrm{ml}$ (unity) or $2 \mu \mathrm{U} / \mathrm{ml}$, and $n$ is an amplification parameter allowing a disproportional rise in $S\left(A_{\mathrm{b}}\right)$ with a doubling of ACTH.

3. Results and Conclusions with Respect to DoseResponse-Time Analysis. The regression of eqs. 33-35 is compatible with data as shown in Fig. 16. The model 


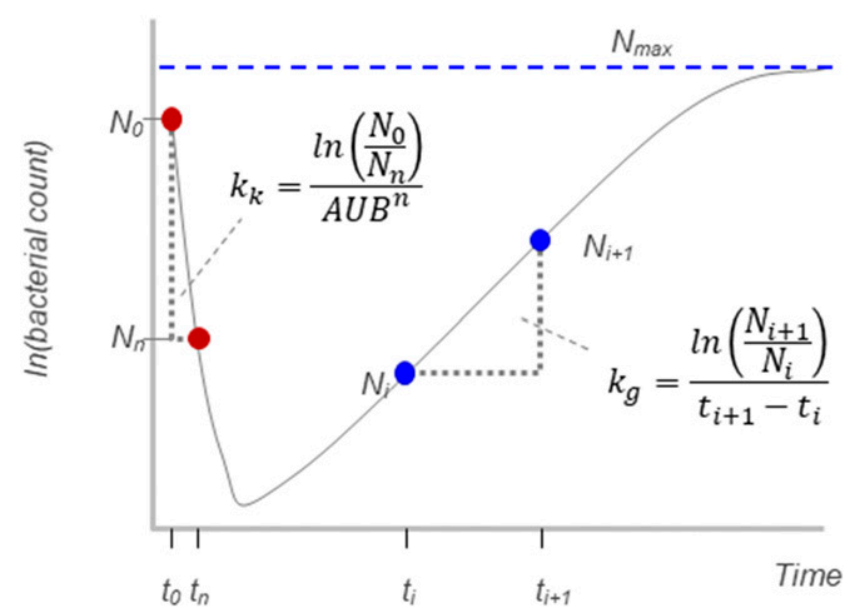

Fig. 15. Semilogarithmic plot of observed (symbols) and model-predicted (lines) response-time data on how to estimate the growth $k_{\mathrm{g}}$ and kill-rate $k_{\mathrm{k}}$ parameters.

is made up of two parallel turnover models that are interconnected, in that the response compartment drives the buildup of the moderator and the change of the moderator is fed back to the response compartment via the loss term of the latter. This mechanism makes up the feedback process that governs the intertwined cortisol-ACTH system. Data show the predose baseline, the initial rapid rise resulting in an overshoot in cortisol release when the ACTH level rises, pharmacodynamic steady-state and the post-exposure rebound. Cortisol levels then decline to a new steady state, which upon lowering of the ACTH exposure plunges to a level below (rebound) the original baseline at about 2. The rebound then oscillates back to baseline at the end of the experimental period. The $k_{\text {out }}$ and $k_{\text {tol }}$ parameters are of the same magnitude $\left(0.16-0.18 \mathrm{~min}^{-1}\right)$, which corresponds to a half-life of cortisol release of about 3 minutes (Table 8).

The biophase exposure to cortisol was assumed, but not confirmed, to be $1 \mu \mathrm{U} / \mathrm{ml}$ at baseline, approximately doubled during the experiment, and then returned back to baseline exposure during washout. This experimental setup allowed us to use a squarewave biophase function for driving the pharmacodynamic response. Data contained information about the model rate constants $k_{\mathrm{in}}, k_{\mathrm{out}}$, and $k_{\mathrm{tol}}$ and the amplification factor $n$. All parameters were estimated with high precision.

4. Pharmacodynamic Interpretation and Comments. The interdependent ACTH-cortisol secretion system is an important and integral part of the hypothalamicpituitary-adrenal axis, responding to stressful conditions and challenges. The studies of Urquhart and Li (1968) used hypophysectomized dogs to maintain control of the ACTH exposure in their perfusion experiments. Interestingly, the overshoot observed in this case study appeared dependent both on dose (exposure) and rate of $\mathrm{ACTH}$ rise in the arterial blood during
TABLE 7

Initial and final parameter estimates and their CV\% $k$ values are given in $\mathrm{h}^{-1}$, and $N$ values are given in colony-forming units.

\begin{tabular}{lccc}
\hline Parameter & Initial Estimate & Final Estimate & CV\% \\
\hline$k_{\mathrm{g}}\left(\mathrm{h}^{-1}\right)$ & & 0.10 & 2 \\
$K_{\mathrm{e}}\left(\mathrm{h}^{-1}\right)$ & 1.0 & 0.77 & 2 \\
$k_{k}\left(\mathrm{~h}^{-1}\right)$ & & 1.0 & 2 \\
$N_{\max }$ & & 37,000 & 6 \\
$N_{01}$ & 11,000 & 3 \\
$N_{02}$ & 11,000 & 3 \\
$N_{03}$ & 12,000 & 4 \\
$N_{04}$ & 13,000 & 5 \\
$N_{05}$ & 11,000 & 3 \\
\hline
\end{tabular}

perfusion (Urquhart and $\mathrm{Li}, 1968$ ). The underlying mechanism(s) was suggested to involve changes in cortisol hydroxylation, and/or other unknown actions of ACTH on steroidogenesis, which is also related to the moderator $M$ in our analysis. We attempted to reanalyze data with either an extended model (according to Ahlström et al., 2011) or an alternative tolerance model (Urquhart and Li, 1968; used in the original analysis). However, neither of these approaches was successful in fully capturing the oscillations. It appears that additional discrete individual data are required to estimate accurate and precise parameters that may account for oscillatory behavior (and tolerance development) in this case.

\section{G. Case Study 7: Dose-Response-Time Analysis of Miotic Effects}

1. Background. Miotic response-time data are an example of the use of DRT analysis when there are no supportive drug concentrations. When we use local applications (e.g., eye drops for drug action in glaucoma), plasma exposure to drug is "downstream" of the biophase containing the pharmacological target and thus cannot serve as a driver of the pharmacological response. This case study therefore illustrates the feasibility of using DRT data in an early preclinical setting in the absence of systemic exposure data.

The miotic response in the cat eye after application of latanoprost (Gabrielsson et al., 2000; Gabrielsson and Weiner, 2016), an ester prodrug analog of prostaglandin $\mathrm{F}_{2 \alpha}$, is assumed to mirror an interaction with prostenoid $\mathrm{F}$ receptors in the smooth muscle of the iris. Thus, in a screening program to find drugs for the treatment of glaucoma, the miotic response in the cat eye was one of the models used to evaluate potential drug candidates. Domestic cats were specially trained to receive eye drops and to allow measurement of the pupillary response. Six animals were used in each of three dose groups. The horizontal diameter of the pupil was measured. The precision of the measurements of the pupil diameter was $1 \mathrm{~mm}(10 \%)$. Figure 17 shows the observed response-time data, and eq. 36 is the biophase model fit to the data. 


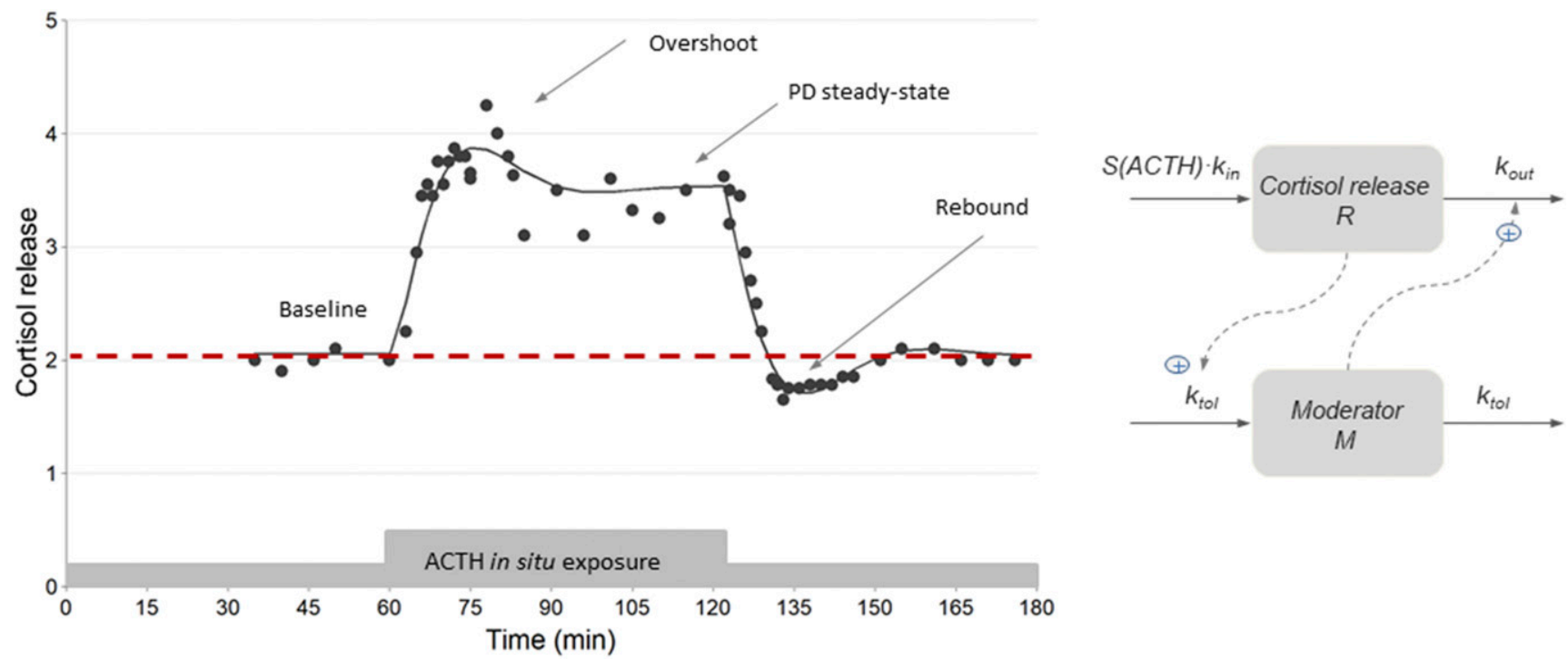

Fig. 16. (Left) Cortisol release rate vs. time in the perfused canine adrenal gland in situ upon stimulation by ACTH (gray bar). Experimental data (solid circles) and the model-predicted curve (solid line) after a doubling of cortisol exposure. The drug provocation is modeled by means of a squarewave function that changes from $1 \mu \mathrm{U} / \mathrm{ml}$ (20-60 minutes) to $2 \mu \mathrm{U} / \mathrm{ml}$ (60-122 minutes), and then back to $1 \mu \mathrm{U} / \mathrm{ml}$ at 122 minutes. (Right) Schematic presentation of the conceptual model used for fitting experimental data of cortisol release rate as a function of ACTH exposure. $R$ denotes the cortisol release rate, $M$ is the moderator, $k_{i n}$ is the turnover rate constant, $k_{\text {out }}$ is the fractional turnover rate constant, and $k_{\text {tol }}$ is the fractional turnover rate constant of the moderator compartment. The stimulatory drug mechanism function is given as a square-wave function with a jump of ACTH exposure from 1 to $2 \mu \mathrm{U} / \mathrm{ml}$ at 60 minutes and then an equally abrupt change back to $1 \mu \mathrm{U} / \mathrm{ml}$ at 122 minutes. PD, pharmacodynamic.

2. Models, Equations, and Exploratory Analysis. The kinetics of latanoprost in the biophase was assumed to be described by a first-order input/output model, including a lag-time:

$$
A_{\mathrm{b}}=\frac{K_{\mathrm{a}} \cdot F \cdot D_{\mathrm{ev}}}{\left(K_{\mathrm{a}}-K\right)}\left[e^{-K \cdot\left(t-t_{\mathrm{lag}}\right)}-e^{-K_{a} \cdot\left(t-t_{\mathrm{lag}}\right)}\right]
$$

$A_{\mathrm{b}}$ is the drug amount in the biophase, $D_{\mathrm{ev}}$ is the actual extravascular dose applied on the cornea, $K_{\mathrm{a}}$ is the firstorder input rate constant, $K$ is the first-order elimination rate constant (assuming $K_{\mathrm{a}}>>K$ ), and $t_{\text {lag }}$ is the lag time during the input of drug into the effect compartment. We also assume that the biophase availability and volume of the biophase compartment are set equal to unity. The biophase function (eq. 36) is then directly driving the response (eq. 37):

$$
E=E_{0}-\frac{I_{\max } \cdot A_{\mathrm{b}}^{n_{\mathrm{H}}}}{\mathrm{ID}_{50}^{n_{\mathrm{H}}}+A_{\mathrm{b}}^{n_{\mathrm{H}}}}
$$

including the baseline value of the contralateral control eye $E_{0}$, the maximum effect $I_{\max }$, the dose at halfmaximal effect $\mathrm{ID}_{50}$, and the sigmoidicity factor $n_{\mathrm{H}} . K_{\mathrm{a}}$, $K, t_{\text {lag }}, I_{\max }, \mathrm{ID}_{50}$, and $n_{\mathrm{H}}$ were then estimated by simultaneously fitting eqs. 36 and 37 to the mean values of the observed effect-time data obtained from each dose (Fig. 18).

3. Results and Conclusions with Respect to DoseResponse-Time Analysis. The treatment data presented here exemplify the use of a biomarker (response) when there are no drug concentration data for kineticl dynamic analyses. We assumed a first-order input/output kinetic model that drives the dynamics. The kinetic and dynamic models were then fit simultaneously to the data to estimate $K_{\mathrm{a}}, K, t_{\mathrm{lag}}, I_{\mathrm{max}}, \mathrm{ID}_{50}$, and $n$ (Table 9 ). The simulated biophase amount versus miotic response is shown in Fig. 18.

4. Pharmacodynamic Interpretation and Comments. This analysis example shows that a well designed experiment that includes only response-time data may nonetheless lend itself to estimation of the underlying kinetic processes without any additional concentrationtime measurements. It also shows that absorption does not have to be instantaneous to enable estimation of biophase kinetics and dynamics. It has been suggested that the cornea acts like a slow-release depot to the anterior segment when latanoprost is topically administered into the eye (Russo et al., 2008), possibly adding to the lag-time effect introduced by the ester conversion of latanoprost to its active species in the eye. The results from a DRT exercise could be used for more refined recommendations regarding dose, dosing interval, and concentration-response sampling times in future (pre)clinical studies. In this particular case and based on human data (Sjöquist and Stjernschantz, 2002), once the drug reaches the systemic circulation, its breakdown is very rapid ( $t_{1 / 2}$ of 17 minutes); thus, this further

TABLE 8

Final parameter estimates

Data are presented as the estimate \pm CV\%.

\begin{tabular}{ccccc}
\hline Parameter & $k_{\text {in }}$ & $k_{\text {out }}$ & $k_{\text {tol }}$ & $n$ \\
\hline \multirow{2}{*}{ Estimate } & concentration $/$ min & $\min ^{-1}$ & $\min ^{-1}$ & \\
\hline
\end{tabular}



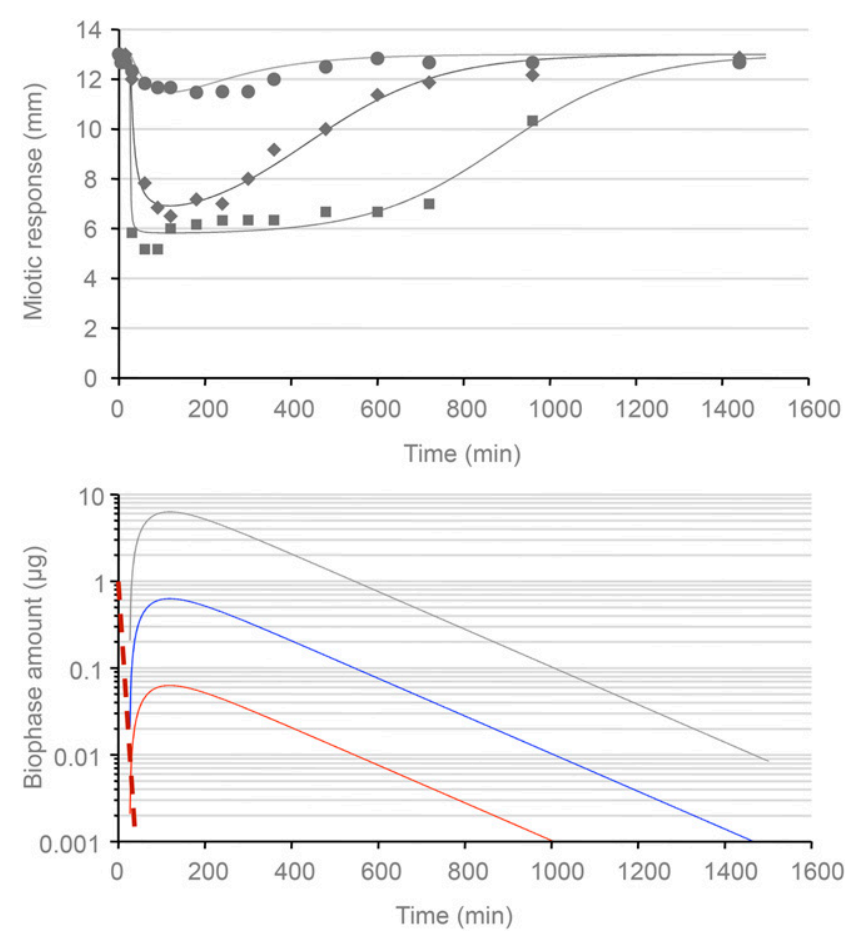

Fig. 17. (Top) Observed (symbols) and model-predicted (lines) responsetime data for latanoprost at the $0.1,1.0$, and $10 \mu \mathrm{g}$ dose levels in the cat eye. (Bottom) Simulated corresponding biophase time courses using final parameter estimates from the regression of the pharmacodynamic model to response-time data in the top panel. The thick dashed red line schematically shows the time course of test compound after intravenous and topical ( $t_{\max } 10$ minutes) dosing onto the cornea, with a plasma halflife of $<10$ minutes in rabbits and cynomolgus monkeys (Sjöquist et al., 1999).

complicates concentration-time-only approaches to modeling. Notably, as the application of latanoprost is in the immediate vicinity of the target biophase, plasma exposure is downstream of the miotic response and therefore not the driver in this situation. Moreover, the fact that the effect precedes (rather than follows) the appearance of drug in the circulation, combined with a prolonged response duration versus a very short plasma half-life of the drug, would make any attempt to correlate the miosis readout to plasma concentration extremely difficult.

\section{H. Case Study 8: Meta-Analysis of Dose-Response- Time Data}

1. Background. The standard pharmacological approach of activating a target around the clock often fails because of time-dependent loss of drug efficacy and postdosing rebound above predose baseline levels. This case study presents data that originally explored the idea that a more comprehensive understanding of the relationship between plasma exposure and a pharmacological response combined with knowledge of the physiologic regulation of metabolism (i.e., simultaneously analyzed considering the effect of key biomarkers) can be used to mitigate these barriers, allowing the invention of new pharmacostrategies

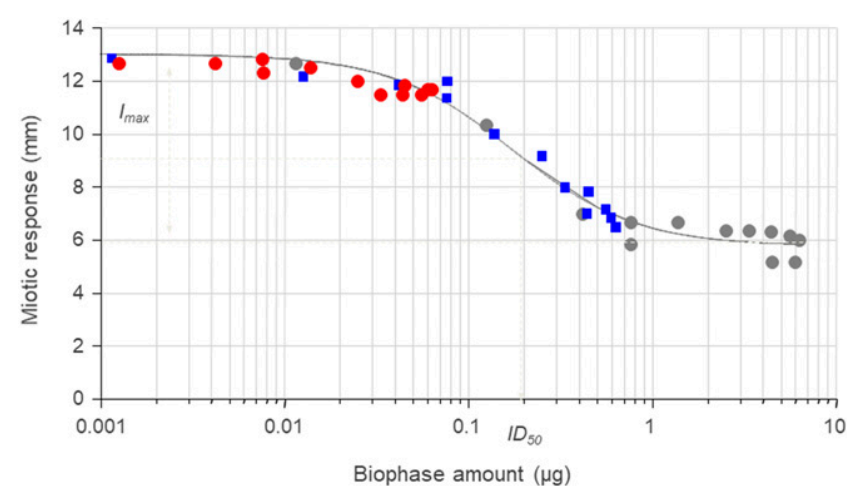

Fig. 18. Observed (symbols) and predicted (solid lines, eq. 37) miotic effect (in millimeters) vs. biophase amount after three different doses of latanoprost (filled red circles, $0.1 \mu \mathrm{g}$; blue squares, $1.0 \mu \mathrm{g}$; and gray circles, $10 \mu \mathrm{g})$. The observed effects are mean values with a coefficient of variation of approximately $30 \%$ for each observation.

(Andersson et al., 2017; Kroon et al., 2017). The metaanalysis aimed at investigating in what way our inference about the system is changed when all drug exposure data are removed. Is it still possible to derive information about pivotal target (FFAs) properties by conducting a DRT data analysis? If not, in what way do the results fail to give insight about system behavior?

This DRT analysis contained the following information: 1) multiple response-time courses of two different biomarkers (FFA and insulin) under acute and semichronic NiAc treatments, conducted in both lean Sprague-Dawley and obese Zucker rats; 2) time delays (between known plasma exposure and biomarker response), feedback patterns (e.g., overshoot and rebound), and two distinct patterns of systemic adaptation prevail (one as a result of insulin control in lean animals, and another pattern due to drug resistance in obese animals); and 3) utilization of a prior exposure-driven analysis, which provided a good opportunity for comparisons between DRT- and exposuredriven analyses. Table 10 contains information about the experimental setup, animals used, administration routes, and study duration, which were all part of the meta-analysis.

2. Results and Conclusions with Respect to DoseResponse-Time Analysis. The meta-analysis of the NiAc-FFA-insulin data challenges several of the previously established constraints to DRT analysis: namely, linear dynamics, stationary system (tolerance/adaptation not allowed), instantaneous equilibrium between plasma and biophase (response), linear plasma kinetics, and so forth. The analysis may also provide a

\section{TABLE 9}

Final parameter estimates

Data are presented as the estimate $\pm \mathrm{CV} \%$. Half-lives of $K_{\mathrm{a}}$ and $K$ are given

\begin{tabular}{ccccccc}
\hline Parameter & $t_{1 / 2 K \mathrm{a}}$ & $t_{1 / 2 K}$ & $t_{\text {lag }}$ & $I_{\max }$ & $\mathrm{ID}_{50}$ & $N$ \\
\hline \multirow{3}{*}{ Estimate } & $35 \pm 30$ & $\min$ & & $m m$ & $\mu g$ & $\min$ \\
& $140 \pm 7$ & $26 \pm 5$ & $7.2 \pm 2$ & $0.17 \pm 10$ & $1.3 \pm 7$
\end{tabular}


TABLE 10

Information about study length, washout profile, dose schedule, route, and nutritional conditions

Number of saline control animals appears within parentheses.

\begin{tabular}{|c|c|c|c|c|c|}
\hline Treatment & Study & Protocol & Route & Lean Rats & Obese Rats \\
\hline \multirow[t]{4}{*}{ Acute } & On-off $1 \mathrm{~h}$ & $1 \mathrm{~h}$ infusion & i.v. & $4(3)$ & $5(3)$ \\
\hline & On-off $5 \mathrm{~h}$ & $5 \mathrm{~h}$ infusion & s.c. & $7(2)$ & $7(5)$ \\
\hline & On-off $12 \mathrm{~h}$ & $12 \mathrm{~h}$ infusion & s.c. & $5(2)$ & $4(2)$ \\
\hline & Step-down $12 \mathrm{~h}$ & $\begin{array}{l}12 \mathrm{~h} \text { infusion plus } 3.5 \mathrm{~h} \text { of } \\
\text { step-down infusion }\end{array}$ & s.c. & $5(3)$ & $4(3)$ \\
\hline Semichronic & Continuous $120 \mathrm{~h}$ & $\begin{array}{l}120 \mathrm{~h} \text { pretreatment infusion } \\
\text { plus } 5 \mathrm{~h} \text { infusion }\end{array}$ & s.c. & $6(2)$ & $8(2)$ \\
\hline
\end{tabular}

framework to conduct meta-analyses of both preclinical and clinical studies when exposure data are scarce or lacking. To our knowledge, multiple mutually interdependent biomarkers confounded by dynamic feedback (e.g., with FFA and insulin, in this case) have not yet been analyzed by means of a DRT approach.

The results were generally well defined system parameters, except for an unacceptably low precision for some of them. By and large, the traditional exposure response and the DRT approaches, however, gave similar results (Table 11). It is also interesting to note that parameter estimates of a very wide numerical range were obtained in the analysis, which probably made the differential equation system rigid. The halflives ranged from a few seconds to more than 100 hours, which is not surprising in a highly regulated metabolic system such as the FFA-insulin interplay. The outcome may help optimize the next study design to increase precision in pivotal parameters. We are aware of the fact that the DRT analysis resulted in some poorly defined parameters (high CV\%), which makes sense in light of particulars of the dataset. Specifically, targeted time ranges will be of greater importance in the design of subsequent studies. However, note the great discrepancy between the half-lives of exposuredriven tolerance versus DRT-driven tolerance in the lean Sprague-Dawley group. The former suggests a long half-life of almost 70 hours, whereas the latter analysis showed a half-life of slightly less than an hour.

3. Pharmacodynamic Interpretation and Comments. The results illustrate the value of applying a quantitative approach to accompany comprehensive physiologic and kinetic-dynamic understanding. A well defined dosing regimen, rate, extent, and timing of drug intervention were designed sequentially across several studies. This resulted in suppression of tolerance and rebound and, most importantly, in profound improvements of the metabolic profile of a preclinical disease model (Andersson et al., 2017; Kroon et al., 2017). In turn, this information may provide vital input to projects aimed at discovery and development of novel drugs for metabolic disorder. As noted by Kroon et al.
(2017), the possibility remains to be investigated whether the response patterns observed with different NiAc administration protocols are exclusive to this agent or may be avoided using other drugs also targeting the HM74/GPR109A receptor. Whether antilipolysis via other targets would display similar response properties to the NiAc HM74/GPR109A receptor interaction is also clearly a worthwhile task for future study. Regardless, this case study is an example of the importance of optimal design of dosing regimens in the treatment of metabolic disorder. Furthermore, the data also emphasize the importance of drug testing in a disease model (obese Zucker rats) compared with control conditions (lean Sprague-Dawley rats), as illustrated not least by the marked difference in tolerance half-life outcome.

\section{Overall Conclusions of the Case Studies}

The eight selected case studies were chosen for their specific data patterns with respect to the onset, intensity, and duration of response, rather than representations of therapeutic class. Whereas case studies 1-7 are representative single-case studies of the aforementioned, case study 8 is an example of applying an extended model and mixed-effects modeling to meta-analysis of several sources of data.

Time series are necessary for a correct assessment of biomarker response data, particularly with time delays between the plasma concentration of test compound and the biomarker response. Since different

TABLE 11

Final estimates of system parameter half-lives from the meta-analysis (biophase- vs. exposure-driven input)

Data are the CV\% and corresponding S.D. for the different parameters.

\begin{tabular}{lccccc}
\hline \multirow{2}{*}{$\begin{array}{l}\text { Parameter, } \\
t_{1 / 2}(\mathrm{~h})\end{array}$} & \multicolumn{2}{c}{ Lean Sprague-Dawley Rats } & & \multicolumn{2}{c}{ Obese Zucker Rats } \\
\cline { 2 - 3 } \cline { 5 - 6 } & DRT Driven & Exposure Driven & & DRT Study & Exposure Driven \\
\hline$k_{\text {out } I}$ & $0.03(16)$ & $0.11(14)$ & & $0.04(45)$ & $0.06(17)$ \\
$k_{\text {tol } I}$ & $0.78(30)$ & $64 .(28)$ & & $4.3(6.0)$ & $5.8(48)$ \\
$k_{\text {out } R I}$ & $0.02(16)$ & $0.01(17)$ & & $28 .(54)$ & $11 .(27)$ \\
$k_{N I}$ & - & - & & $5.0(170)$ & $29 .(35)$ \\
$k_{\text {out } F}$ & $0.001(1)$ & $0.002(140)$ & & $0.002(150)$ & $0.004(120)$ \\
$k_{\text {tol } F}$ & $0.36(1000)$ & $0.57(67)$ & & $1.2(4.3)$ & $1.1(24)$ \\
$k_{\text {out } R F}$ & $0.08(300)$ & $0.71(29)$ & & $77(58)$ & $41(38)$ \\
$k_{N F}$ & $140(330)$ & $110(65)$ & & $190(24)$ & $18(14)$ \\
\hline
\end{tabular}


model parameters contribute to varying extents at specific phases (onset, intensity, and duration) of a pharmacological response, close scrutiny of the experimental protocol becomes an integral part of design. A full characterization of determinants behind the onset, intensity, and duration of response often requires multiple routes, rates, and extents of dosing regimens.

DRT analyses typically use more parameters (e.g., biophase availability, biophase rate constant) than plasma concentration-driven models, since the driver (biophase) of pharmacological response has to be predicted as such. Plasma half-lives are also typically much shorter than are pharmacodynamic responses (e.g., shown in case studies 3 and 8). Finally, DRT models are generally compound, biomarker, and species dependent and are therefore difficult to generalize within and across therapeutic classes. Table 12 lists characteristics, mechanisms, and features, along with some suggestions for improvements to experimental design, applied to the case studies discussed in this article.

\section{Discussion}

\section{A. Background}

This review focuses on DRT data analysis when knowledge on test compound (drug) exposure is scarce or entirely lacking. We start by dissecting the different phases of a pharmacodynamic response-time course (Fig. 2), and we propose what parameters may be related. We then move on to exemplify structures of biophase functions from the literature (Fig. 3). These range from a simple bolus input coupled to monoexponential decline and first-order and saturable input/output rates to parallel exogenous and endogenous input functions. We provide a brief mathematical description of biophase, drug mechanism, and commonly applied turnover functions. We also discuss alternative rate models and put them into a biologic perspective. This review contains eight case studies. These case studies were selected to demonstrate how to tackle baseline/no baseline, time delays, peak shifts, saturation, cell growth/kill data, functional adaptation, rebound, multiple sources of drug provocations and biomarkers, and rapid exposure-response data (Table 2).

Isaksson et al. (2009) and Andersson et al. $(2016,2017)$ successfully performed meta-analyses on preclinical data on NiAc (nonlinear absorption and elimination)-induced fatty acid lowering, with accurate and precise parameter estimates. The kinetic nonlinearities were well separated from the pharmacodynamic nonlinearities. Pharmacological data of two intertwined biomarkers and of multiple doses, rates, routes, and schedules were simultaneously regressed. This attests to the view that the success of a DRT analysis is a matter of good experimental design (Gabrielsson and Weiner, 2010, 2016; Andersson et al., 2016) rather than the number of observations, as was recently suggested (González-Sales et al., 2017).

The applicability of DRT analyses in both preclinical and clinical situations is illustrated in Table 13, which compiles more than 60 examples found in a thorough search of the literature. We also list some situations in which DRT analyses may be potentially useful but may be challenging (Table 14).

To this end, references listed in Table 13 represent a compilation of reports published up to and including 2017. References to abstracts and oral communications have intentionally been excluded from this review. These studies cover a large range of therapeutic and experimental areas. Some of the preclinical studies use two or more modes of administration and are more experimental in nature (Schoenwald and Smolen, 1971; Smolen, 1971a,b, 1976a,b, 1978; Smolen et al., 1972; Smolen and Weigand, 1973; Isaksson et al., 2009; Andersson et al., 2017). Other studies are performed in critically ill patients (Salem et al., 2016), in neonates (Trefz et al., 2015), under disease progression (Musuamba et al., 2015), for postoperative pain management (Abou Hammoud et al., 2009), in drug interaction studies (Gruwez et al., 2005, 2007), and in metabolic systems (Fasanmade and Jusko, 1995; Hamberg et al., 2010; Ternant et al., 2014; Saffian et al., 2016), to mention just a few.

\section{B. What Do Different Doses, Routes, and Rates of Input Add?}

When different doses (see case study 1) are given in vivo, the process of onset of action, potential response peak shifts with increasing doses, saturation of response at higher doses, and functional adaptation of duration of action may be observed in the pharmacological response-time courses. When different routes of administration (e.g., intravenous and subcutaneous; see case study 1) are applied, the biophase availability may be estimated separately from the other kinetic and dynamic parameters. Nonlinearities in biophase kinetics may also be revealed (case study 8).

When different doses and routes are tested simultaneously, information about saturation of either the absorption or disposition of drug or both processes may be discerned from saturable pharmacodynamic processes (Andersson et al., 2017). Functional adaptation may be observed and quantified when repeated dosing is done (case study 2; Andersson et al., 2016, 2017). The two routes of administration in case study 2 also revealed absorption-rate-limited elimination in the pharmacodynamic data.

Neither the pharmacokinetics nor the pharmacodynamics need to be linear first order, time invariant, or instantaneous or display a constant baseline. A metaanalysis of complex response-time data of fatty acids (FFAs) and insulin turnover in plasma after multiple provocations of NiAc clearly demonstrated the full 


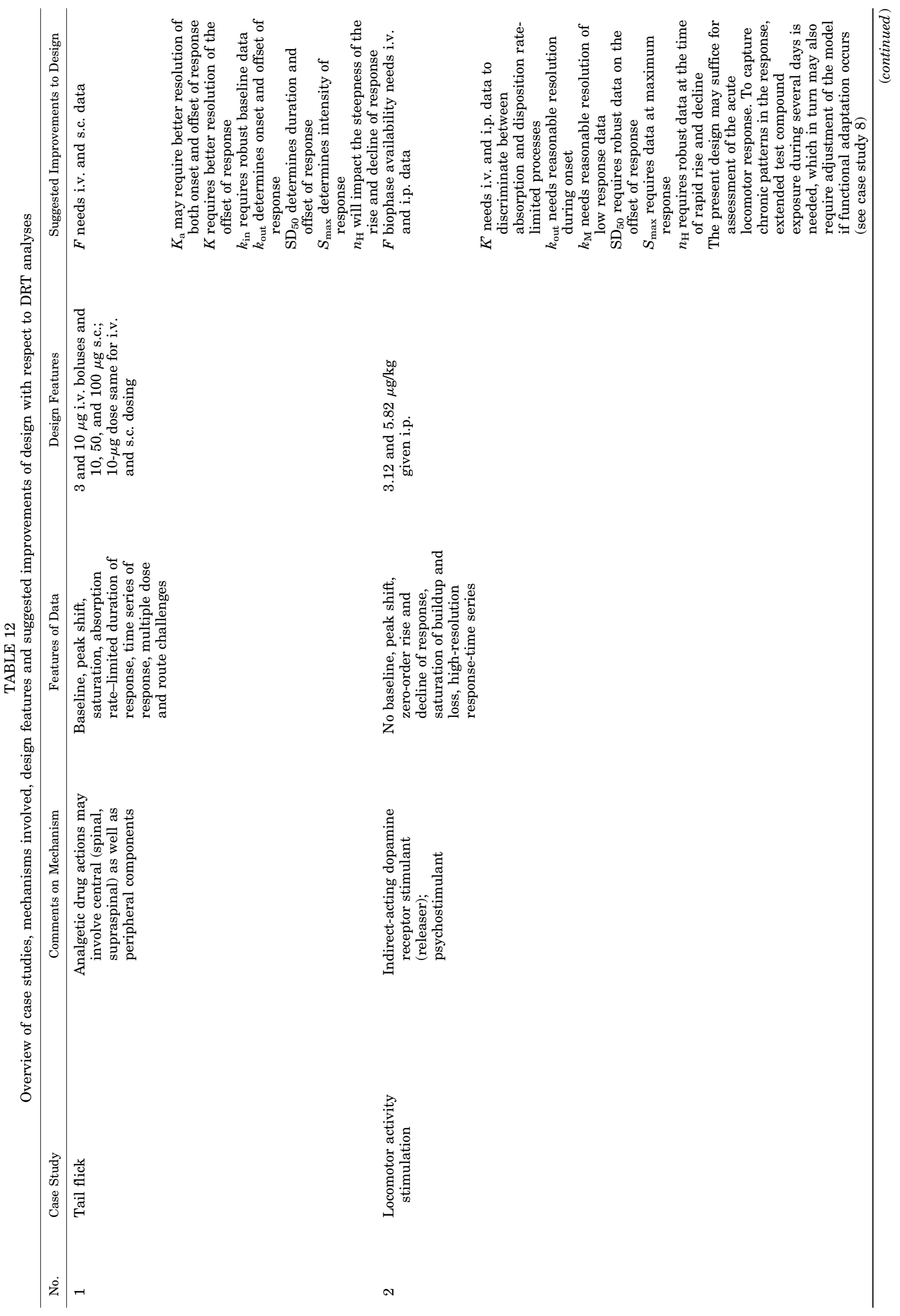



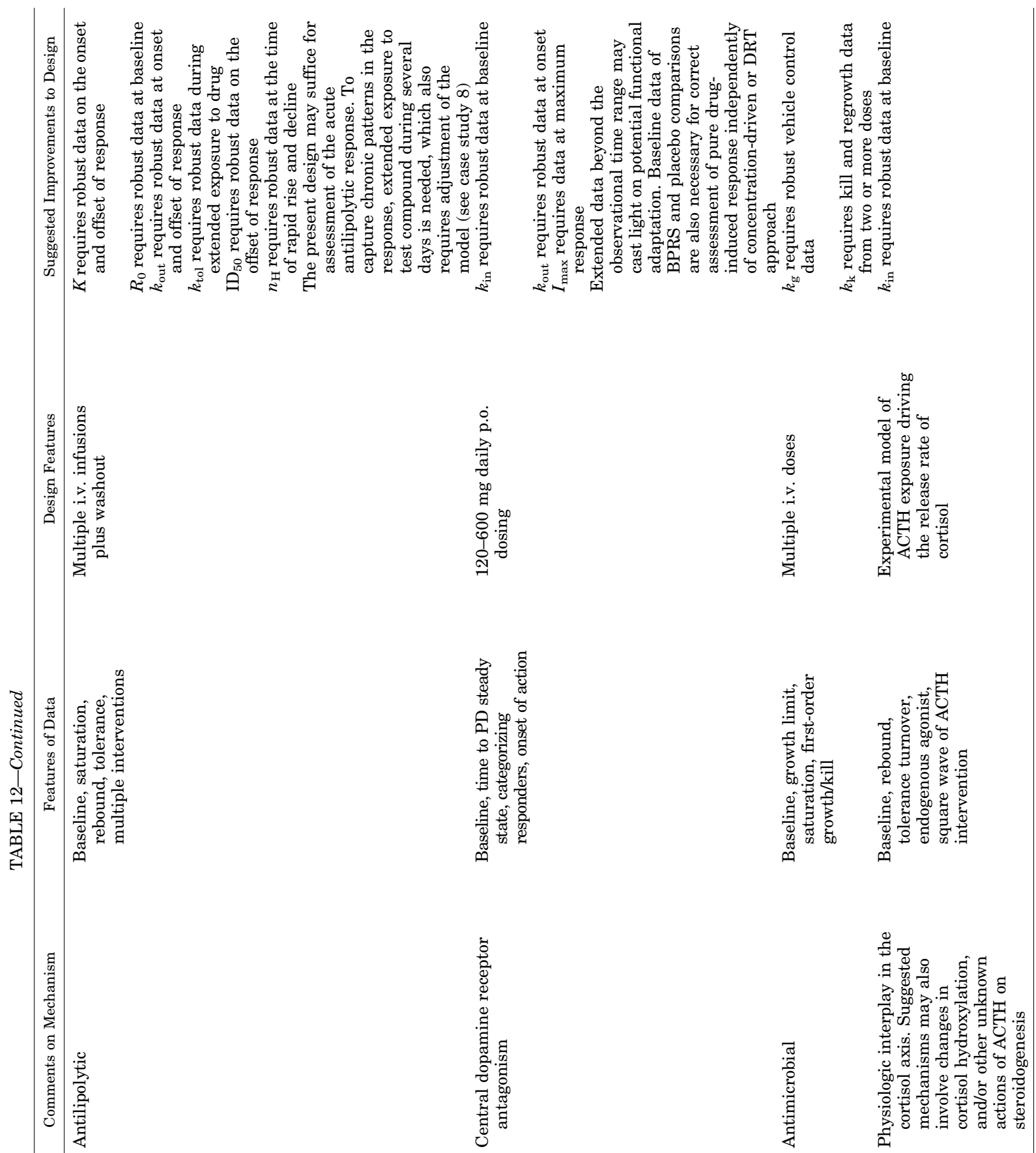

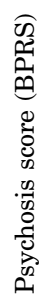

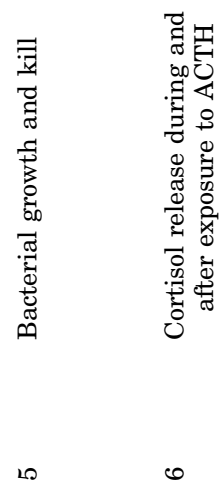

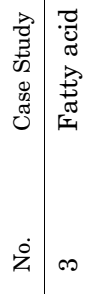
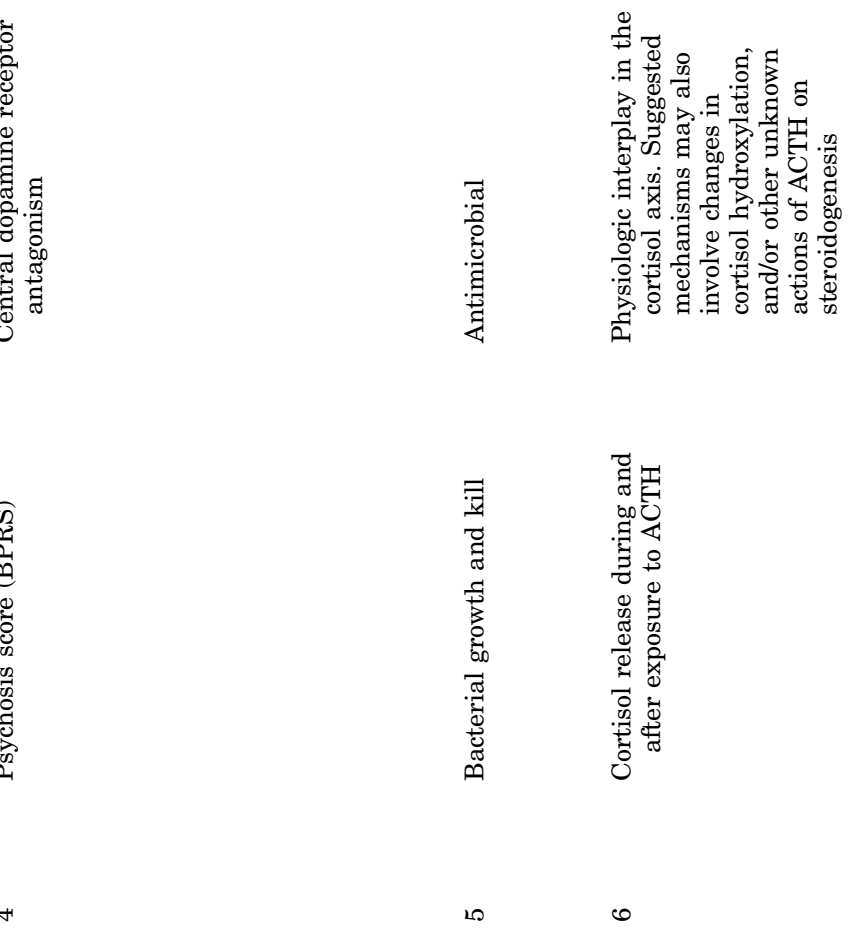


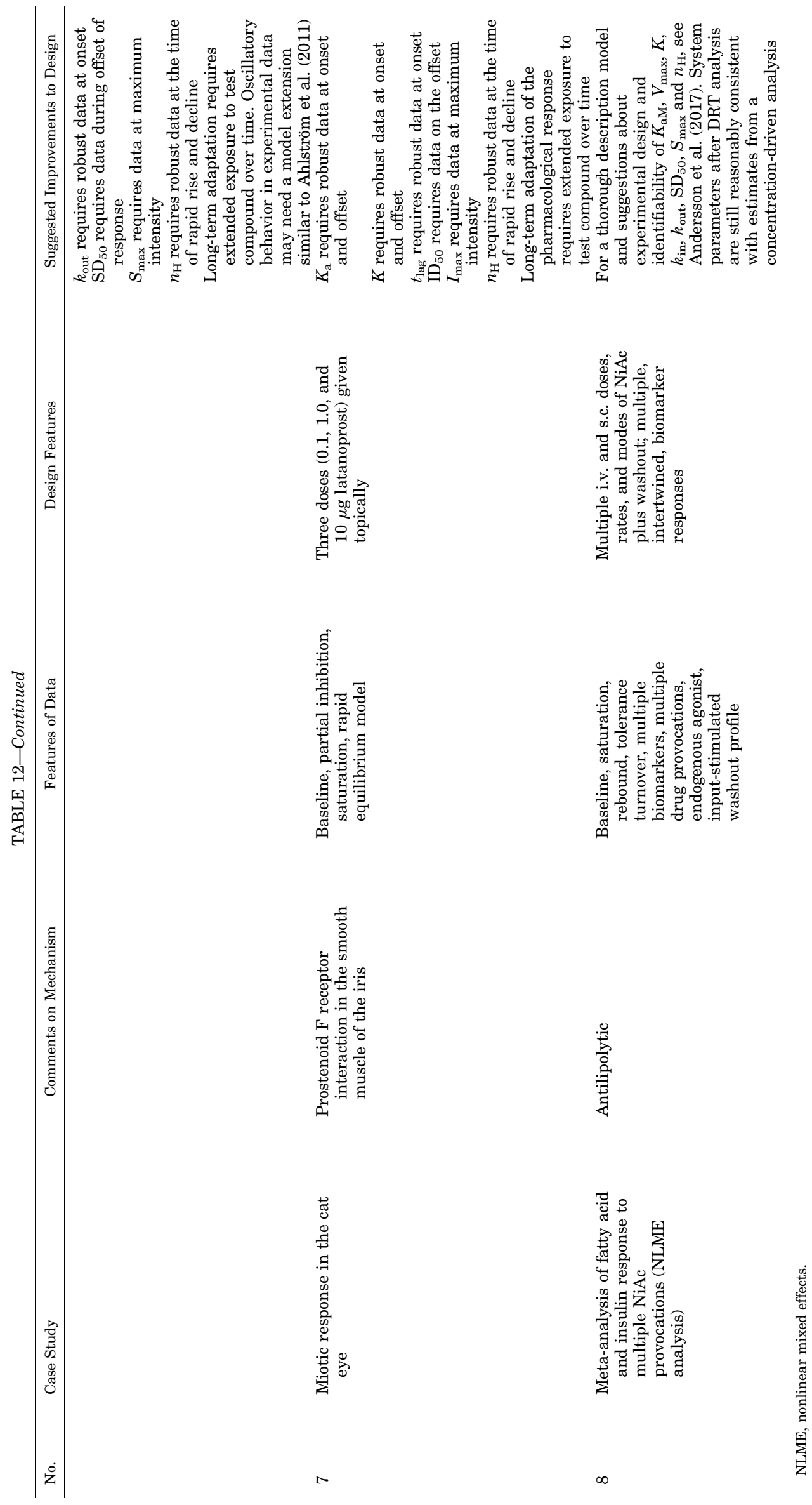




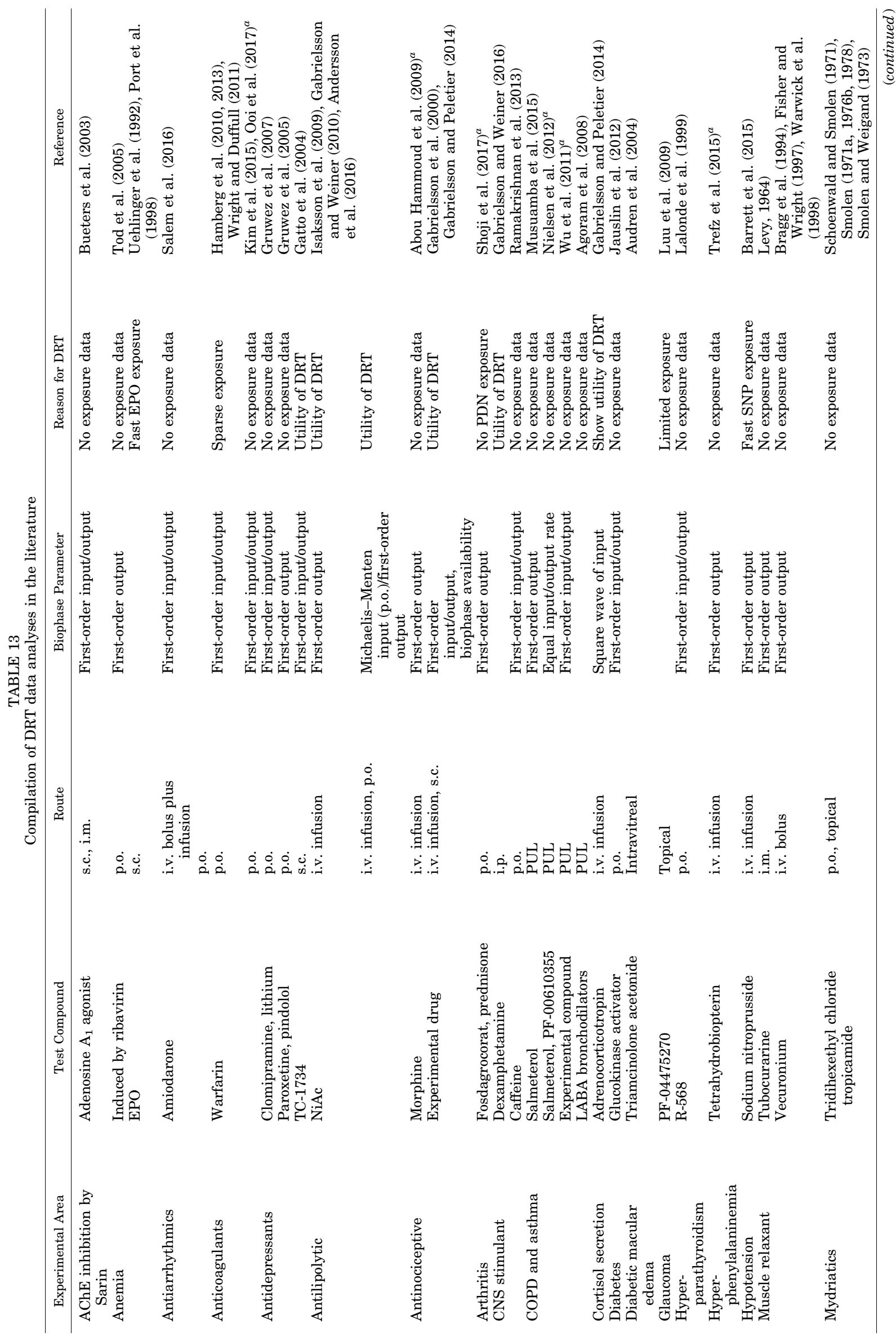




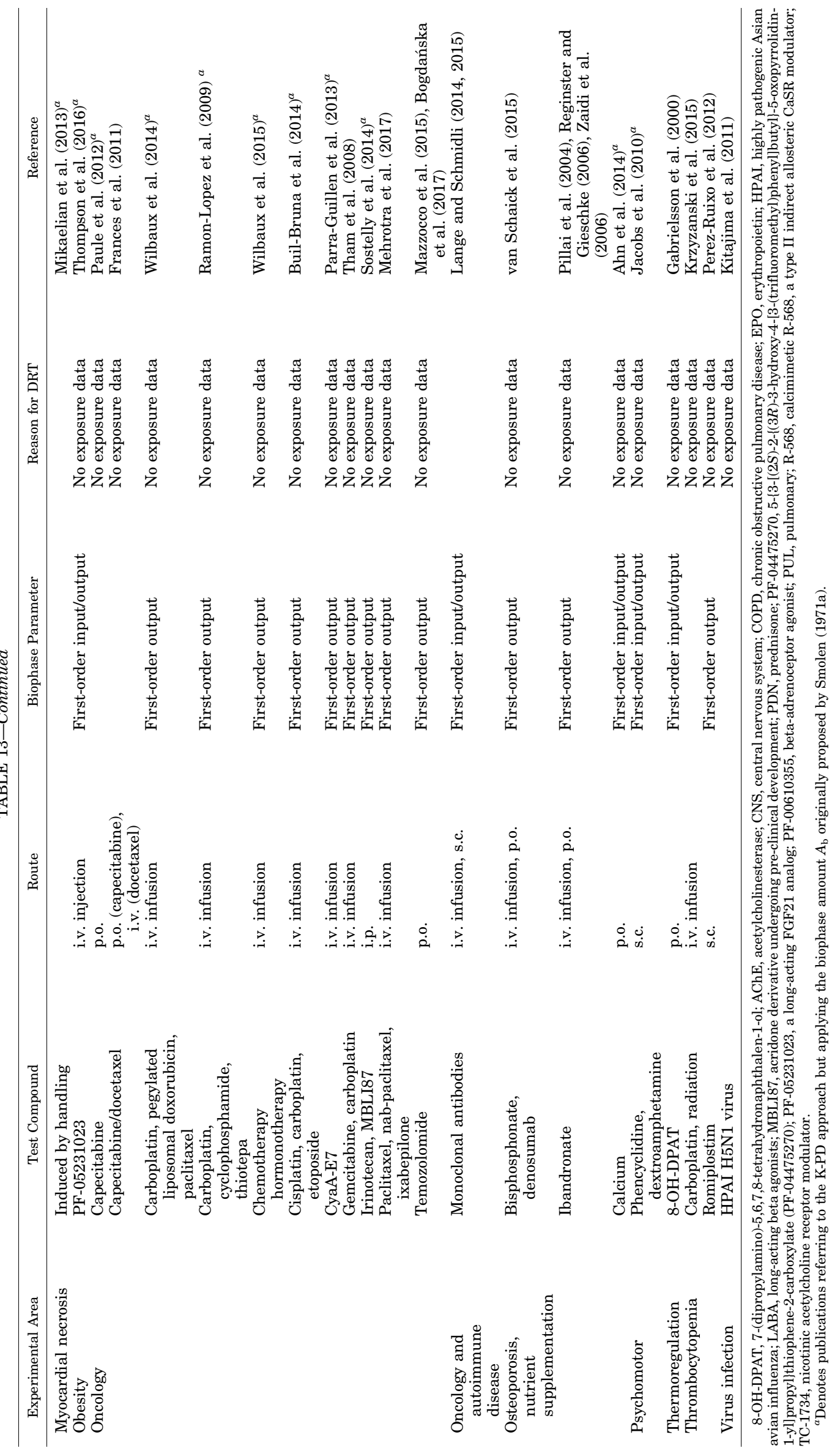


Gabrielsson et al.

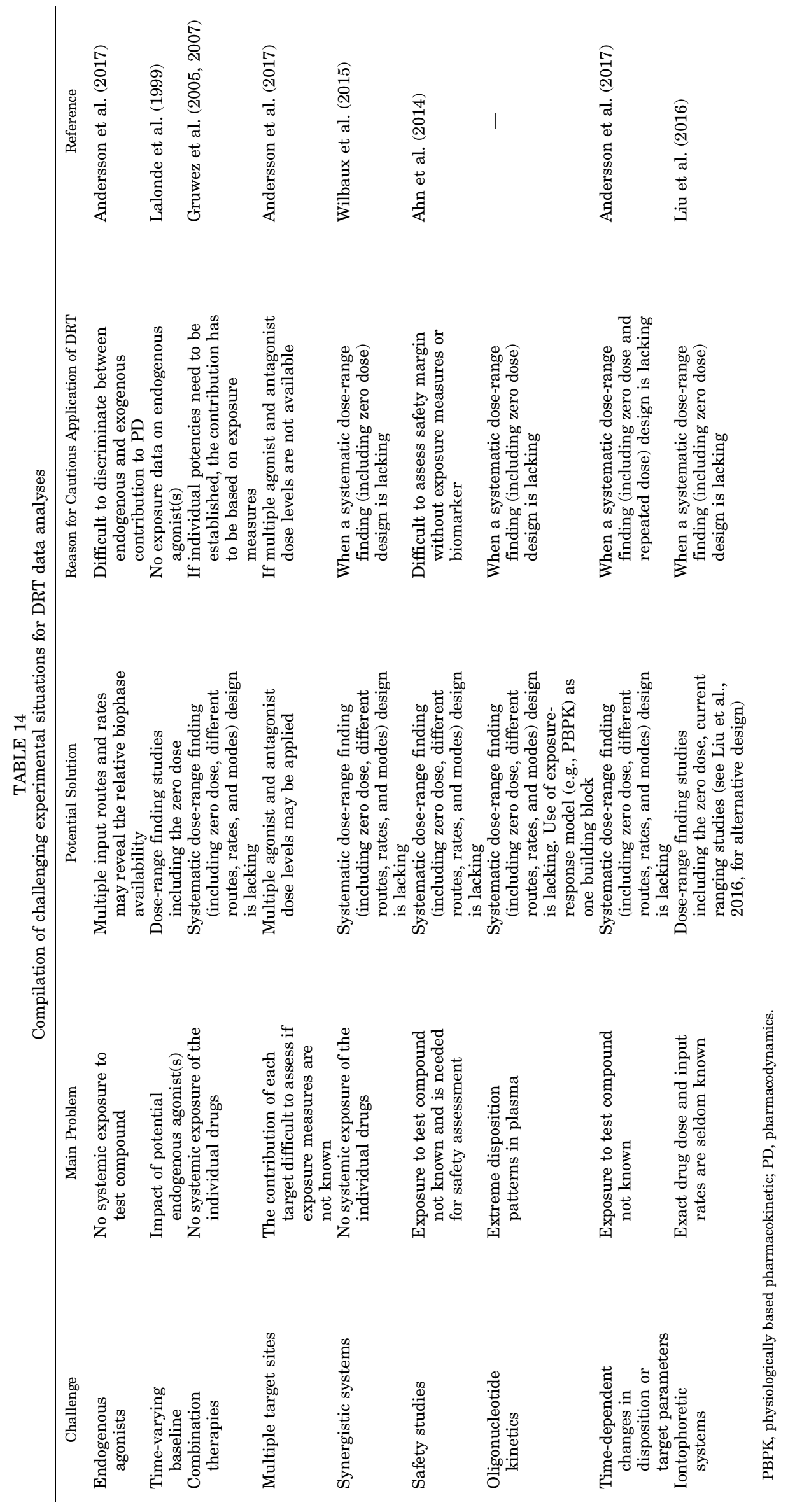

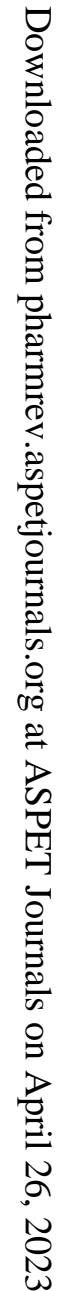


capacity of the DRT approach (Isaksson et al., 2009; Andersson et al., 2017). Not only were the estimated system parameters $\left(k_{\text {out }}, k_{\text {tol }}\right.$, etc.) similar to those obtained from a full exposure-driven analysis, but the interaction between FFAs and insulin was also correctly captured. Simultaneous sampling of multiple interacting biomarkers (e.g., FFAs and insulin) offers an opportunity to take the analysis to an even higher level and deconvolute interaction patterns between the biomarkers, but still keep the assessment focus on both drug properties (e.g., $\mathrm{ED}_{50}$ ) and target properties (e.g., $k_{\text {in }}$ and $k_{\text {out }}$ ) (Andersson et al., 2017). The use of multiple sources of biomarker data obtained from diverse patterns of drug (NiAc) intervention allowed accurate and precise parameter estimates. The mechanistic model may therefore have a higher translational potential.

Some of the preclinical studies use two or more modes of administration and are more experimental in nature (Schoenwald and Smolen, 1971; Smolen, 1971a,b, 1976a,b, 1978; Smolen et al., 1972; Smolen and Weigand, 1973; Isaksson et al., 2009; Gabrielsson et al., 2015; Andersson et al., 2017). Smolen (1971a, 1976b) described a model for bioavailability and biokinetic behavior of a mydriatic drug. He extended the analysis to application of DRT data for bioequivalence testing. Others have applied a systems analysis approach (Veng-Pedersen and Modi, 1993). Fasanmade and Jusko (1995) developed a kinetic/dynamic model using a hypothetical reactive metabolite to explain formation of methemoglobin. These studies clearly demonstrate the potential of DRT analyses in a preclinical setting and their applications to drug discovery data. There are several clinical examples of how DRT modeling is applied to analyze (sparse) human data (see Table 13). The statistical methodology of DRT modeling is elegantly illustrated with data from clinical studies of therapeutic biologics in autoimmune disease (Lange and Schmidli, 2014, 2015), and Salem et al. (2016) applied a DRT approach to critically ill patients where traditional exposure-driven approaches are difficult and/or unethical. The drug mechanism function was driven by the biophase amount rather than the rate. Trefz et al. (2015) developed a pharmacodynamic DRT model to improve the description of individual sensitivity to tetrahydrobiopterin responsiveness in neonates with hyperphenylalaninemia. Abou Hammoud et al. (2009) demonstrated how a DRT model, which characterizes the time course of morphine-induced analgesia in the immediate postoperative period, could be used for pain management. Wilbaux et al. (2015) successfully applied a DRT approach for the assessment of treatment efficacy in metastatic castration-resistant prostate cancer, using the count of circulating tumor cells (CTCs) as a promising surrogate marker. The model is the first to quantify the dynamics of prostate-specific antigen and CTC count during treatment in patients with metastatic castration-resistant prostate cancer.
It has great potential as a general framework for showing the combination effects of chemotherapy in other cancer types to compare the properties of CTCs.

Gruwez et al. (2005, 2007) developed a DRTinteraction model between selective serotonin reuptake inhibitors and pindolol for the assessment of clinical treatment of depression (Montgomery-Åsberg Depression Rating Scale score). They concluded that the DRT model of Montgomery-Åsberg Depression Rating Scale score kinetics was able to capture individual data of a clinical trial and to yield estimates of the typical values and the interindividual variability of the parameters. A clinical trial simulation based on the model illustrated how it might be used as a tool for clinical trial planning in the field of research on antidepressants and adjunctive treatments.

Ahn et al. (2014) used a semimechanistic approach to characterize the $\mathrm{Ca}^{2+}$-parathyroid hormone (PTH) system after intake of thermal spring water containing calcium or calcium carbonate tablets. Without noticeable differences in plasma $\mathrm{Ca}^{2+}$ levels, the changes in PTH responses were used as a surrogate marker for calcium absorption. With a more enhanced study design, it is believed that their approach can be applied to evaluate calcium absorption, as determined by PTHtime responses.

A recent review on challenge tests of reaction induced by Sephadex, interleukin $1 \beta$, collagen, lipopolysaccharide, and glucose and changes caused in eosinophil count, interleukin 6, paw-swelling, tumor necrosis factor $\alpha$, and blood glucose, respectively, applied the DRT approach as a substitute to mimic time courses of the challenging agents (Sephadex, collagen, lipopolysaccharide, and glucose) when exposure to these challengers was lacking (Gabrielsson et al., 2015). Table 14 shows some demanding experimental situations that identify problems related to DRT analyses, with literature references to potential solutions.

\section{Drug Delivery by Means of Iontophoresis}

Drug administration through the skin has been investigated for several decades, and many delivery techniques have been explored to facilitate drug permeation across this outer bodily barrier. Among these, iontophoresis is a novel promising drug delivery system, which enhances skin penetration and the release rate of a number of drugs that are otherwise poorly permeable through the skin (for a review, see Iredahl et al., 2015). DRT analyses may play an important role in iontophoretic systems due to their time- and concentration-wise detachment from systemic exposure, although information that fully characterizes the rate and extent of drug input is still a key factor that needs to be sorted out prior to proposing a biophase structure. However, promising results were recently collected during histamine iontophoresis with laser Doppler monitoring (Liu et al., 2016). This approach allowed a fixed dose of histamine 
to be delivered and an objective, continuous, and dynamic measurement of histamine epicutaneous response to be recorded in children and adults. The reduced data set was then analyzed by means of a DRT model of the local histamine response.

\section{Permutations of Smolen's Model: The Kinetic- dynamic K-PD Rate of Change Model}

DRT analyses were pioneered by Smolen and Levy in the 1960 s and 1970 s with a series of classic papers paving some of the theoretical ground (Levy, 1971; Schoenwald and Smolen, 1971; Smolen, 1971a,b; Smolen et al., 1972; Smolen and Weigand, 1973). A different permutation, denoted kinetic-dynamic K-PD, was more recently suggested (Jacqmin et al., 2007). The latter bolus model suggests that the elimination rate from the biophase $k \cdot A_{\mathrm{b}}$ drives the pharmacological response rather than the biophase amount $\left(A_{\mathrm{b}}\right.$ or exposure) per se (eq. 38).

$$
\left\{\begin{array}{c}
\frac{d A_{\mathrm{b}}}{d t}=-k \cdot A_{\mathrm{b}} \\
S\left(k \cdot A_{\mathrm{b}}\right)=1+\frac{S_{\max } \cdot\left(k \cdot A_{\mathrm{b}}\right)}{\mathrm{SD}_{50}+\left(k \cdot A_{\mathrm{b}}\right)} \\
\frac{d R}{d t}=k_{\text {in }} \cdot S\left(k \cdot A_{\mathrm{b}}\right)-k_{\text {out }} \cdot R
\end{array}\right.
$$

The $\mathrm{SD}_{50}$ is the rate of change of drug in the biophase that results in half-maximal drug-induced response, which makes this parameter difficult to interpret and compare across species. There are few examples in the pharmacological literature where the rate of change in biophase amount rather than the biophase amount per se, is the "driver" of the pharmacological response. The division of $\mathrm{SD}_{50}$ by $k$ in eq. 38 (middle line) may then cause a parameter identifiability problem.

In contrast to the biophase bolus model in K-PD, there are numerous examples in the literature (Table 1) and case studies $1-3,7$, and 8 that demonstrate more elaborate structures. Several authors refer to the K-PD $\left(k \cdot A_{\mathrm{b}}\right)$ approach but have in fact applied the classic Smolen biophase kinetics $\left(A_{\mathrm{b}}\right)$ for driving the pharmacodynamic model (Table 13; see footnoted references).

\section{E. General Conclusions}

This review aims to raise cross-research and development discipline awareness about DRT data analyses to explore and improve interpretation of data. Although the DRT methodology is not new, it is still comparatively rare in experimental and clinical pharmacology. This review examines how the time course of a pharmacological response can be dissected to gain further insight. It emphasizes the significance of time courses, illustrates the importance of multiple dose information, and addresses issues in the examination of timeresponse relationships. Furthermore, this review shows how quantitative pharmacology adds significant value to target turnover information and why ignoring this may cause misleading results and conclusions. In conclusion, access to robust and discrete baseline data (absolute numbers rather than differences or other conversions) from multiple doses, routes, rates, and administration modes will provide the best prerequisite for fruitful DRT analysis.

Smolen et al. (1972) stated that

The use of pharmacological data for pharmacokinetic systems analysis would be obviously limited if responses were restricted to only those effects that can be directly observed and utilized without further, or only minimal, treatment. When an assay for a drug in biological media does not exist or is difficult to perform, it can be considered that the development of a pharmacological method could instead provide the most expeditious means of accomplishing a pharmacokinetic analysis of the drug's behavior.

He also suggested that "the use of pharmacological data for bioavailability studies should be considered irrespective of whether an assay method for the drug exists. The decision to use either or both types of data should depend on the relative sensitivity precision, convenience, and economy" Smolen (1976b). It is our hope that our account will further underscore his sentiments, and that the examples and suggested solutions in this article will inspire (and renew interest in) DRT analysis of pharmacodynamic responses.

\section{Authorship Contributions}

Participated in research design: Gabrielsson, Andersson, Jirstrand, Hjorth.

Conducted experiments: Gabrielsson.

Performed data analysis: Gabrielsson, Andersson.

Wrote or contributed to the writing of the manuscript: Gabrielsson, Andersson, Jirstrand, Hjorth.

\section{References}

Abou Hammoud H, Simon N, Urien S, Riou B, Lechat P, and Aubrun F (2009) Intravenous morphine titration in immediate postoperative pain management: population kinetic-pharmacodynamic and logistic regression analysis. Pain 144: 139-146.

Agoram BM, Milligan PA, and van der Graaf PH (2008) A non-parametric method to analyse time-course of effect in the absence of pharmacokinetic data: application to inhaled bronchodilators. Eur J Pharm Sci 34:250-256.

Ahlström C, Peletier LA, and Gabrielsson J (2011) Quantitative analysis of rate and extent of tolerance of biomarkers: application to nicotinic acid-induced changes in non-esterified fatty acids in rats. Eur $J$ Pharm Sci 44:250-264.

Ahn JE, Jeon S, Lee J, Han S, and Yim DS (2014) Modeling of the parathyroid hormone response after calcium intake in healthy subjects. Korean $J$ Physiol Pharmacol 18:217-223.

Andersson R, Jirstrand M, Peletier L, Chappell MJ, Evans ND, and Gabrielsson J (2016) Dose-response-time modelling: second-generation turnover model with integral feedback control. Eur J Pharm Sci 81:189-200.

Andersson R, Kroon T, Almquist J, Jirstrand M, Oakes ND, Evans ND, Chappel MJ, and Gabrielsson J (2017) Modeling of free fatty acid dynamics: insulin and nicotinic acid resistance under acute and chronic treatments. $J$ Pharmacokinet Pharmacodyn 44:203-222.

Audren F, Tod M, Massin P, Benosman R, Haouchine B, Erginay A, Caulin C, Gaudric A, and Bergmann JF (2004) Pharmacokinetic-pharmacodynamic modeling of the effect of triamcinolone acetonide on central macular thickness in patients with diabetic macular edema. Invest Ophthalmol Vis Sci 45:3435-3441.

Barrett JS, Hirankarn S, Holford N, Hammer GB, Drover DR, Cohane CA, Anderson B, Dombrowski E, Reece T, Zajicek A, et al. (2015) A hemodynamic model to guide 
blood pressure control during deliberate hypotension with sodium nitroprusside in children. Front Pharmacol 6:151.

Bogdańska MU, Bodnar M, Belmonte-Beitia J, Murek M, Schucht P, Beck J and Pérez-García VM (2017) A mathematical model of low grade gliomas treated with temozolomide and its therapeutical implications. Math Biosci 288:1-13.

Bragg P, Fisher DM, Shi J, Donati F, Meistelman C, Lau M, and Sheiner LB (1994) Comparison of twitch depression of the adductor pollicis and the respiratory muscles. Pharmacodynamic modeling without plasma concentrations. Anesthesiology 80:310-319.

Bueters TJ, Joosen MJ, van Helden HP, Ijzerman AP, and Danhof M (2003) Adenosine A1 receptor agonist N6-cyclopentyladenosine affects the inactivation of acetylcholinesterase in blood and brain by sarin. J Pharmacol Exp Ther 304: 1307-1313.

Buil-Bruna N, López-Picazo JM, Moreno-Jiménez M, Martín-Algarra S, Ribba B, and Trocóniz IF (2014) A population pharmacodynamic model for lactate dehydrogenase and neuron specific enolase to predict tumor progression in small cell lung cancer patients. AAPS J 16:609-619.

Calabrese EJ (2008) Addiction and dose response: the psychomotor stimulant theory of addiction reveals that hormetic dose responses are dominant. Crit Rev Toxicol 38:599-617.

Dafters R and Taggart P (1992) Biotelemetric investigation of morphine's thermic and kinetic effects in rats. Psychopharmacology (Berl) 106:195-201.

Fasanmade AA and Jusko WJ (1995) An improved pharmacodynamic model for formation of methemoglobin by antimalarial drugs. Drug Metab Dispos 23: $573-576$

Fisher DM and Wright PM (1997) Are plasma concentration values necessary for pharmacodynamic modeling of muscle relaxants? Anesthesiology 86:567-575.

Frances N, Claret L, Bruno R, and Iliadis A (2011) Tumor growth modeling from clinical trials reveals synergistic anticancer effect of the capecitabine and docetaxel combination in metastatic breast cancer. Cancer Chemother Pharmacol 68: $1413-1419$

Gabrielsson J and Hjorth S (2012) Quantitative Pharmacology: An Introduction to Integrative Pharmacokinetic-Pharmacodynamic Analysis, 1st ed, Swedish Pharmaceutical Press, Stockholm, Sweden.

Gabrielsson J and Hjorth S (2016) Pattern recognition in pharmacodynamic data analysis. AAPS J 18:64-91.

Gabrielsson J and Hjorth S (2018) Integration of pharmacokinetic and pharmacodynamic reasoning and its importance in drug discovery, in Early Drug Development: Bringing a Preclinical Candidate to the Clinic (Giordanetto F ed), 1st ed, pp 369-400, Wiley-VCH Verlag GmbH \& Co. KGaA, Weinheim, Germany.

Gabrielsson J, Hjorth S, Vogg B, Harlfinger S, Gutierrez PM, Peletier L, Pehrson R, and Davidsson P (2015) Modeling and design of challenge tests: inflammatory and metabolic biomarker study examples. Eur J Pharm Sci 67:144-159.

Gabrielsson J, Jusko WJ, and Alari L (2000) Modeling of dose-response-time data: four examples of estimating the turnover parameters and generating kinetic functions from response profiles. Biopharm Drug Dispos 21:41-52.

Gabrielsson J and Peletier LA (2008) A flexible nonlinear feedback system that captures diverse patterns of adaptation and rebound. AAPS J 10:70-83.

Gabrielsson J and Peletier LA (2014) Dose-response-time data analysis involving nonlinear dynamics, feedback and delay. Eur J Pharm Sci 59:36-48.

Gabrielsson J, Peletier LA, and Hjorth S (2018) In vivo potency revisited - Keep the target in sight. Pharmacol Ther 184:177-188.

Gabrielsson J and Weiner D (2010) Pharmacokinetic and Pharmacodynamic Data Analysis: Concepts and Applications, 4th ed, Swedish Pharmaceutical Press, Stockholm, Sweden.

Gabrielsson J and Weiner D (2016) Pharmacokinetic and Pharmacodynamic Dato Analysis: Concepts and Applications, 5th ed, Swedish Pharmaceutical Press, Stockholm, Sweden.

Gatto GJ, Bohme GA, Caldwell WS, Letchworth SR, Traina VM, Obinu MC, Laville M, Reibaud M, Pradier L, Dunbar G, et al. (2004) TC-1734: an orally active neuronal nicotinic acetylcholine receptor modulator with antidepressant, neuroprotective and long-lasting cognitive effects. CNS Drug Rev 10:147-166.

González-Sales M, Nekka F, Tanguay M, Tremblay PO, and Li J (2017) Modelling the dose-response relationship: the fair share of pharmacokinetic and pharmacodynamic information. Br J Clin Pharmacol 83:1240-1251.

Gruwez B, Dauphin A, and Tod M (2005) A mathematical model for paroxetine antidepressant effect time course and its interaction with pindolol. $J$ Pharmacokinet Pharmacodyn 32:663-683.

Gruwez B, Poirier MF, Dauphin A, Olié JP, and Tod M (2007) A kineticpharmacodynamic model for clinical trial simulation of antidepressant action: application to clomipramine-lithium interaction. Contemp Clin Trials 28: $276-287$

Hamberg AK, Friberg LE, Hanséus K, Ekman-Joelsson BM, Sunnegårdh J, Jonzon A, Lundell B, Jonsson EN, and Wadelius M (2013) Warfarin dose prediction in children using pharmacometric bridging--comparison with published pharmacogenetic dosing algorithms. Eur J Clin Pharmacol 69:1275-1283.

Hamberg AK, Wadelius M, Lindh JD, Dahl ML, Padrini R, Deloukas P, Rane A, and Jonsson EN (2010) A pharmacometric model describing the relationship between warfarin dose and INR response with respect to variations in CYP2C9, VKORC1, and age. Clin Pharmacol Ther 87:727-734.

Iredahl F, Sadda V, Ward LJ, Hackethal J, Farnebo S, Tesselaar E, and Sjöberg F (2015) Modeling perfusion dynamics in the skin during iontophoresis of vasoactive drugs using single-pulse and multiple-pulse protocols. Microcirculation 22: 446-453.

Isaksson C, Gabrielsson J, Wallenius K, Peletier LA, and Toreson H (2009) Turnover modeling of non-esterified fatty acids in rats after multiple intravenous infusions of nicotinic acid. Dose Response 7:247-269.

Jacobs T, Straetemans R, Molenberghs G, Adriaan Bouwknecht J, and Bijnens L (2010) A latent pharmacokinetic time profile to model dose-response survival data. J Biopharm Stat 20:759-767.
Jacqmin P, Snoeck E, van Schaick EA, Gieschke R, Pillai P, Steimer JL, and Girard P (2007) Modelling response time profiles in the absence of drug concentrations: definition and performance evaluation of the K-PD model. $J$ Pharmacokinet Pharmacodyn 34:57-85.

Jauslin PM, Karlsson MO, and Frey N (2012) Identification of the mechanism of action of a glucokinase activator from oral glucose tolerance test data in type 2 diabetic patients based on an integrated glucose-insulin model. J Clin Pharmacol 52:1861-1871.

Kim S, Gaweda AE, Wu D, Li L, Rai SN, and Brier ME (2015) Simplified warfarin dose-response pharmacodynamic models. Biomed Eng (Singapore) 27:1550001

Kitajima M, Huang Y, Watanabe T, Katayama H, and Haas CN (2011) Dose-response time modelling for highly pathogenic avian influenza A (H5N1) virus infection. Lett Appl Microbiol 53:438-444.

Kroon T, Baccega T, Olsén A, Gabrielsson J, and Oakes ND (2017) Nicotinic acid timed to feeding reverses tissue lipid accumulation and improves glucose control in obese Zucker rats[S]. J Lipid Res 58:31-41.

Krzyzanski W, Perez-Ruixo JJ, and Harrold J (2015) Pharmacodynamic model for chemoradiotherapy-induced thrombocytopenia in mice. J Pharmacokinet Pharmacodyn 42:709-720.

Lalonde RL, Gaudreault J, Karhu DA, and Marriott TB (1999) Mixed-effects modeling of the pharmacodynamic response to the calcimimetic agent R-568. Clin Pharmacol Ther 65:40-49.

Lange MR and Schmidli H (2014) Optimal design of clinical trials with biologics using dose-time-response models. Stat Med 33:5249-5264.

Lange MR and Schmidli H (2015) Analysis of clinical trials with biologics using dosetime-response models. Stat Med 34:3017-3028

Levine SZ and Leucht S (2010) Elaboration on the early-onset hypothesis of antipsychotic drug action: treatment response trajectories. Biol Psychiatry 68:86-92.

Levy G (1964) Relationship between rate of elimination of tubocurarine and rate of decline of its pharmacological activity. Br J Anaesth 36:694-695.

Levy G (1971) Kinetics of drug action in man. Acta Pharmacol Toxicol (Copenh) 29 (Suppl 3):203-210

Lewander T, Westerbergh SE, and Morrison D (1990) Clinical profile of remoxipride--a combined analysis of a comparative double-blind multicentre trial programme. Acta Psychiatr Scand Suppl 358:92-98.

Liu X, Jones BL, Roberts JK, and Sherwin CM (2016) Population pharmacokinetic/pharmacodynamic modeling of histamine response measured by histamine iontophoresis laser Doppler. J Pharmacokinet Pharmacodyn 43:385-393.

Luu KT, Zhang EY, Prasanna G, Xiang C, Anderson S, Fortner J, and Vicini P (2009) Pharmacokinetic-pharmacodynamic and response sensitization modeling of the intraocular pressure-lowering effect of the EP4 agonist $5-\{3-[(2 S)-2-\{(3 R)-3-$ hydroxy-4-[3-(trifluoromethyl)phenyl]butyl\}-5-oxopyrrolidin-1-yl]propyl)thiophene2-carboxylate (PF-04475270). J Pharmacol Exp Ther 331:627-635.

Marques TR, Arenovich T, Agid O, Sajeev G, Muthén B, Chen L, Kinon BJ, and Kapur S (2011) The different trajectories of antipsychotic response: antipsychotics versus placebo. Psychol Med 41:1481-1488.

Mazzocco P, Barthélémy C, Kaloshi G, Lavielle M, Ricard D, Idbaih A, Psimaras D, Renard MA, Alentorn A, Honnorat J, et al. (2015) Prediction of response to temozolomide in low-grade glioma patients based on tumor size dynamics and genetic characteristics. CPT Pharmacometrics Syst Pharmacol 4:728-737.

Mehrotra S, Sharma MR, Gray E, Wu K, Barry WT, Hudis C, Winer EP, Lyss AP, Toppmeyer DL, Moreno-Aspitia A, et al. (2017) Kinetic-pharmacodynamic model of chemotherapy-induced peripheral neuropathy in patients with metastatic breast cancer treated with paclitaxel, nab-paclitaxel, or ixabepilone: CALGB 40502 (Alliance). AAPS J 19:1411-1423.

Mikaelian I, Dunn ME, Mould DR, Hirkaler G, Geng W, Coluccio D, Nicklaus R, Singer T, and Reddy M (2013) Differential analysis of transient increases of serum cTnI in response to handling in rats. Pharmacol Res Perspect 1:e00011.

Musuamba FT, Teutonico D, Maas HJ, Facius A, Yang S, Danhof M, and Della Pasqua O (2015) Prediction of disease progression, treatment response and dropout in chronic obstructive pulmonary disease (COPD). Pharm Res 32:617-627.

Nielsen JC, Hutmacher MM, Cleton A, Martin SW, and Ribbing J (2012) Longitudinal FEV1 dose-response model for inhaled PF-00610355 and salmeterol in patients with chronic obstructive pulmonary disease. J Pharmacokinet Pharmacodyn 39:619-634.

Ooi QX, Wright DFB, Tait RC, Isbister GK, and Duffull SB (2017) A joint model for vitamin K-dependent clotting factors and anticoagulation proteins. Clin Pharmacokinet 56:1555-1566.

Parra-Guillen ZP, Berraondo P, Ribba B, and Trocóniz IF (2013) Modeling tumor response after combined administration of different immune-stimulatory agents. $J$ Pharmacol Exp Ther 346:432-442.

Paule I, Tod M, Hénin E, You B, Freyer G, and Girard P (2012) Dose adaptation of capecitabine based on individual prediction of limiting toxicity grade: evaluation by clinical trial simulation. Cancer Chemother Pharmacol 69:447-455.

Perez-Ruixo JJ, Green B, Doshi S, Wang YM, and Mould DR (2012) Romiplostim dose response in patients with immune thrombocytopenia. J Clin Pharmacol 52: $1540-1551$

Pillai G, Gieschke R, Goggin T, Jacqmin P, Schimmer RC, and Steimer JL (2004) A semimechanistic and mechanistic population PK-PD model for biomarker response to ibandronate, a new bisphosphonate for the treatment of osteoporosis. $\mathrm{Br}$ J Clin Pharmacol 58:618-631.

Port RE, Ding RW, Fies T, and Schärer K (1998) Predicting the time course of haemoglobin in children treated with erythropoietin for renal anaemia. $\mathrm{Br} J \mathrm{Clin}$ Pharmacol 46:461-466.

Ramakrishnan S, Rajaraman S, Laxminarayan S, Wesensten NJ, Kamimori GH, Balkin TJ, and Reifman J (2013) A biomathematical model of the restoring effects of caffeine on cognitive performance during sleep deprivation. $J$ Theor Biol 319.23-33.

Ramon-Lopez A, Nalda-Molina R, Valenzuela B, and Perez-Ruixo JJ (2009) Semimechanistic model for neutropenia after high dose of chemotherapy in breast cancer patients. Pharm Res 26:1952-1962. 
Reginster JY and Gieschke R (2006) Clinical utility of a pharmacostatistical model for ibandronate in postmenopausal osteoporosis. Curr Drug Metab 7:827-836.

Russo A, Riva I, Pizzolante T, Noto F, and Quaranta L (2008) Latanoprost ophthalmic solution in the treatment of open angle glaucoma or raised intraocular pressure: a review. Clin Ophthalmol 2:897-905.

Saffian SM, Duffull SB, Roberts RL, Tait RC, Black L, Lund KA, Thomson AH, and Wright DF (2016) Influence of genotype on warfarin maintenance dose predictions produced using a Bayesian dose individualization tool. Ther Drug Monit 38:677-683.

Salem JE, El-Aissaoui M, Alazard M, Hulot JS, Aissaoui N, Le-Heuzey JY, FunckBrentano C, Faisy C, and Urien S (2016) Modeling of amiodarone effect on heart rate control in critically ill patients with atrial tachyarrhythmias. Clin Pharmacokinet 55:991-1002.

Sawynok J and Liu J (2014) Contributions of peripheral, spinal, and supraspinal actions to analgesia. Eur J Pharmacol 734:114-121.

Schoenwald RD and Smolen VF (1971) Drug-absorption analysis from pharmacological data. II. Transcorneal biophasic availability of tropicamide. J Pharm Sci 60: 1039-1045.

Shoji S, Suzuki A, Conrado DJ, Peterson MC, Hey-Hadavi J, McCabe D, Rojo R, and Tammara BK (2017) Dissociated agonist of glucocorticoid receptor or prednisone for active rheumatoid arthritis: effects on P1NP and osteocalcin pharmacodynamics. CPT Pharmacometrics Syst Pharmacol 6:439-448.

Sjöquist B and Stjernschantz J (2002) Ocular and systemic pharmacokinetics of latanoprost in humans. Surv Ophthalmol 47 (Suppl 1):S6-S12.

Sjöquist B, Tajallaei S, and Stjernschantz J (1999) Pharmacokinetics of latanoprost in the cynomolgus monkey. 1st communication: single intravenous, oral or topical administration on the eye. Arzneimittelforschung 49:225-233.

Smolen VF (1971a) Quantitative determination of drug bioavailability and biokinetic behavior from pharmacological data for ophthalmic and oral administrations of a mydriatic drug. J Pharm Sci 60:354-365.

Smolen VF (1971b) Determination of time course of in vivo pharmacological effects from in vitro drug-release testing. J Pharm Sci 60:878-882.

Smolen VF (1976a) Theoretical and computational basis for drug bioavailability determinations using pharmacological data. I. General considerations and procedures. J Pharmacokinet Biopharm 4:337-353.

Smolen VF (1976b) Theoretical and computational basis for drug bioavailability determinations using pharmacological data. II. Drug input in equilibrium to response relationships. J Pharmacokinet Biopharm 4:355-375.

Smolen VF (1978) Bioavailability and pharmacokinetic analysis of drug responding systems. Annu Rev Pharmacol Toxicol 18:495-522.

Smolen VF, Barile RG, and Theophanous TG (1972) Relationship between dose, ef fect, time, and biophasic drug levels. J Pharm Sci 61:467-470.

Smolen VF and Weigand WA (1973) Drug bioavailability and pharmacokinetic analysis from pharmacological data. J Pharmacokinet Biopharm 1:329-336.

Sostelly A, Payen L, Guitton J, Di Pietro A, Falson P, Honorat M, Boumendjel A, Gèze A, Freyer G, and Tod M (2014) Quantitative evaluation of the combination between cytotoxic drug and efflux transporter inhibitors based on a tumour growth inhibition model. Fundam Clin Pharmacol 28:161-169.

Stauffer V, Case M, Kollack-Walker S, Ascher-Svanum H, Ball T, Kapur S, and Kinon BJ (2011) Trajectories of response to treatment with atypical antipsychotic medication in patients with schizophrenia pooled from 6 double-blind, randomized clinical trials. Schizophr Res 130:11-19.

Ternant D, Büchler M, Thibault G, Ohresser M, Watier H, Lebranchu Y, and Paintaud G (2014) Influence of FcyRIIIA genetic polymorphism on T-lymphocyte depletion induced by rabbit antithymocyte globulins in kidney transplant patients. Pharmacogenet Genomics 24:26-34.

Tham LS, Wang L, Soo RA, Lee SC, Lee HS, Yong WP, Goh BC, and Holford NH (2008) A pharmacodynamic model for the time course of tumor shrinkage by gemcitabine + carboplatin in non-small cell lung cancer patients. Clin Cancer Res 14:4213-4218.

Thompson WC, Zhou Y, Talukdar S, and Musante CJ (2016) PF-05231023, a longacting FGF21 analogue, decreases body weight by reduction of food intake in nonhuman primates. J Pharmacokinet Pharmacodyn 43:411-425.

Tod M, Farcy-Afif M, Stocco J, Boyer N, Bouton V, Sinègre M, and Marcellin P (2005) Pharmacokinetic/pharmacodynamic and time-to-event models of ribavirin-induced anaemia in chronic hepatitis C. Clin Pharmacokinet 44:417-428.

Trefz F, Lichtenberger O, Blau N, Muntau AC, Feillet F, Bélanger-Quintana A, van Spronsen F, and Munafo A (2015) Tetrahydrobiopterin (BH4) responsiveness in neonates with hyperphenylalaninemia: a semi-mechanistically-based, nonlinear mixed-effect modeling. Mol Genet Metab 114:564-569.

Uehlinger DE, Gotch FA, and Sheiner LB (1992) A pharmacodynamic model of erythropoietin therapy for uremic anemia. Clin Pharmacol Ther 51:76-89.

Urquhart J and Li CC (1968) The dynamics of adrenocortical secretion. Am J Physiol 214:73-85.

van Rossum JM and van Koppen AT (1968) Kinetics of psycho-motor stimulant drug action. Eur J Pharmacol 2:405-408.

van Schaick E, Zheng J, Perez Ruixo JJ, Gieschke R, and Jacqmin P (2015) A semimechanistic model of bone mineral density and bone turnover based on a circular model of bone remodeling. $J$ Pharmacokinet Pharmacodyn 42:315-332.

Veng-Pedersen P and Modi NB (1993) Application of neural networks to pharmacodynamics. J Pharm Sci 82:918-926.

Warwick NR, Graham GG, and Torda TA (1998) Pharmacokinetic analysis of the effect of vecuronium in surgical patients: pharmacokinetic and pharmacodynamic modeling without plasma concentrations. Anesthesiology 88:874-884.

Wilbaux M, Hénin E, Oza A, Colomban O, Pujade-Lauraine E, Freyer G, Tod M and You B (2014) Prediction of tumour response induced by chemotherapy using modelling of CA-125 kinetics in recurrent ovarian cancer patients. Br J Cancer 110:1517-1524.

Wilbaux M, Tod M, De Bono J, Lorente D, Mateo J, Freyer G, You B, and Hénin E (2015) A joint model for the kinetics of CTC count and PSA concentration during treatment in metastatic castration-resistant prostate cancer. CPT Pharmacometrics Syst Pharmacol 4:277-285.

Wright DF and Duffull SB (2011) Development of a bayesian forecasting method for warfarin dose individualization. Pharm Res 28:1100-1111.

Wu K, Looby M, Pillai G, Pinault G, Drollman AF, and Pascoe S (2011) Population pharmacodynamic model of the longitudinal FEV1 response to an inhaled longacting anti-muscarinic in COPD patients. $J$ Pharmacokinet Pharmacodyn 38: 105-119.

Zaidi M, Epstein S, and Friend K (2006) Modeling of serum C-telopeptide levels with daily and monthly oral ibandronate in humans. Ann N Y Acad Sci 1068:560-563. 